Florida International University

FIU Digital Commons

FIU Electronic Theses and Dissertations

University Graduate School

$11-15-2019$

\title{
Charged Fusion Product Plasma Diagnostics in Spherical Tokamaks
}

Alexander Netepenko

anete001@fiu.edu

Follow this and additional works at: https://digitalcommons.fiu.edu/etd

Part of the Plasma and Beam Physics Commons

\section{Recommended Citation}

Netepenko, Alexander, "Charged Fusion Product Plasma Diagnostics in Spherical Tokamaks" (2019). FIU Electronic Theses and Dissertations. 4306.

https://digitalcommons.fiu.edu/etd/4306

This work is brought to you for free and open access by the University Graduate School at FIU Digital Commons. It has been accepted for inclusion in FIU Electronic Theses and Dissertations by an authorized administrator of FIU Digital Commons. For more information, please contact dcc@fiu.edu. 


\section{FLORIDA INTERNATIONAL UNIVERSITY}

Miami, Florida

\section{CHARGED FUSION PRODUCT PLASMA DIAGNOSTICS IN SPHERICAL TOKAMAKS}

A dissertation submitted in partial fulfillment of

the requirements for the degree of

DOCTOR OF PHILOSOPHY

in

PHYSICS

by

Alexander Netepenko

2019 
To: Dean Michael R. Heithaus

College of Arts, Sciences and Education

This dissertation, written by Alexander Netepenko, and entitled Charged Fusion Product Plasma Diagnostics in Spherical Tokamaks, having been approved in respect to style and intellectual content, is referred to you for judgment.

We have read this dissertation and recommend that it be approved.

$\begin{array}{r}\hline \text { Jose R. Almirall } \\ \hline \text { Brian A. Raue } \\ \hline \text { Joerg Reinhold } \\ \hline \text { Werner U. Boeglin, Major Professor }\end{array}$

Date of Defense: November 15, 2019

The dissertation of Alexander Netepenko is approved.

Dean Michael R. Heithaus

College of Arts, Sciences and Education

Andrés G. Gil

Vice President for Research and Economic Development and Dean of the University Graduate School

Florida International University, 2019 
(C) Copyright 2019 by Alexander Netepenko

All rights reserved. 


\section{DEDICATION}

I would like to dedicate this dissertation to my grandfather, Vitaly Netepenko, who worked hard from the very young age to support his family and didn't have an opportunity to receive an education, and who always wanted me to have a better life and achieve that goal of becoming an educated person. 


\section{ACKNOWLEDGMENTS}

I would like to express a gratitude to my advisor, Dr. Werner Boeglin, for his guidance and help in my dissertation research and for sharing his knowledge and expertise in broad field of plasma physics.

I would like to say thank you to my dissertation committee: Dr. Jose Almirall, Dr. Brian Raue and Dr. Joerg Reinhold for their participation in my graduate research and their valuable feedback throughout my graduate program years.

I also would like to say special thank you to Dr. Rajamani Narayanan for his help in learning and understanding the plasma confinement and magnetohydrodynamics theoretical aspects.

This work has been supported by the Department of Energy grant DE-SC0001157. 


\section{ABSTRACT OF THE DISSERTATION \\ CHARGED FUSION PRODUCT PLASMA DIAGNOSTICS IN SPHERICAL \\ TOKAMAKS \\ by}

Alexander Netepenko

Florida International University, 2019

Miami, Florida

Professor Werner U. Boeglin, Major Professor

Charged fusion products from the $\mathrm{D}(\mathrm{d}, \mathrm{p}) \mathrm{t}$ reaction, protons $(\mathrm{p})$ and tritons $(\mathrm{t})$, can be detected to obtain time and position dependent information on the fusion reaction profile in plasmas heated with neutral particles beams. We have developed a prototype instrument consisting of six charged particle detectors with ion-implanted-silicon surface barrier detectors. Each detector is combined with two replaceable collimators in such a way that it can accept $3 \mathrm{MeV}$ protons and $1 \mathrm{MeV}$ tritons emitted from a well-defined area inside the plasma. The detectors thus provide curved views across the plasma volume.

Combining the data of all six detectors allows one to study changes in the reaction profile with an expected time resolution of about $1 \mathrm{~ms}$. These changes are mainly because of slow variations in the neutral beam density profile, as well as rapid changes resulting from MHD instabilities. With an envisioned energy resolution of $100-150 \mathrm{keV}$ it will be also possible to study proton energy spectra containing the information on the plasma fast ion distribution function. The anticipated proton energy spectra have been calculated analytically as well as modeled using Monte Carlo methods. 
The effect of the fusion reaction cross-section anisotropy on the observed rates have been analyzed as well as the effect of the magnetic field on the detector acceptance has been studied. The emissivity profile has been modeled for a specific plasma discharge from the MAST 2013 experimental campaign using the PPPL code TRANSP including a recent model for fast ion transport. The calculated proton rates have been compared to the measured ones to investigate the origin of a large discrepancy between experimental data and standard TRANSP results.

A graphic user interface was developed for optimizing detector orientation and data analysis. Furthermore, the new hardware was implemented in the online data acquisition system and its general performance and capabilities were improved. 


\section{TABLE OF CONTENTS}

CHAPTER

PAGE

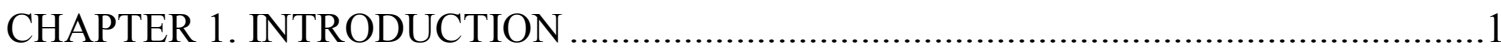

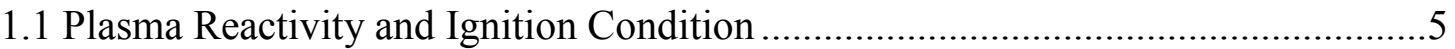

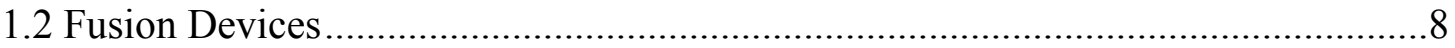

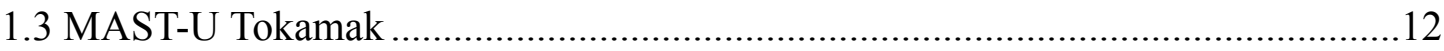

1.4 Single Particle Motion and Drift Velocities........................................................15

1.5 The Good and the Bad Magnetic Field Curvature ..................................................17

CHAPTER 2. PLASMA MAGNETO HYDRO DYNAMIC (MHD) EQUILIBRIUM ...19

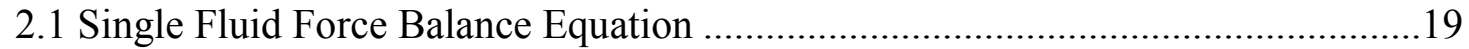

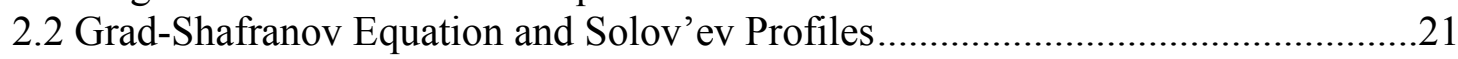

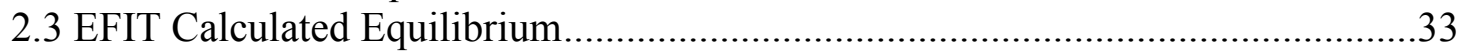

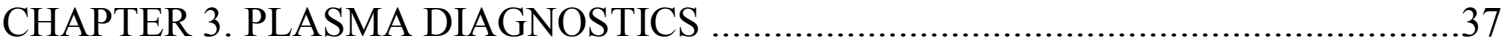

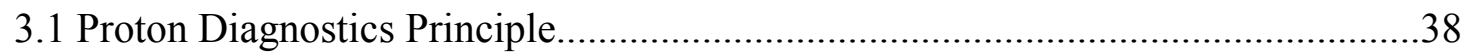

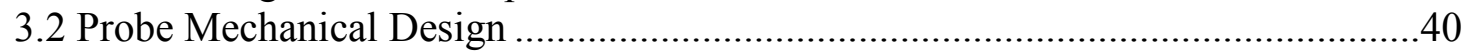

3.3 Effect of Trajectory Curvature Inside the Detector Housing ...................................48

CHAPTER 4. DETECTOR ACCEPTANCE AND RATE PREDICTION .......................55

4.1 Acceptance Reduction Due to a Magnetic Field......................................................57

4.2 Rate Calculation Based on TRANSP Modeled Emissivity Profile ........................61

4.3 Rate Calculation Approach Analytical Justification.............................................65

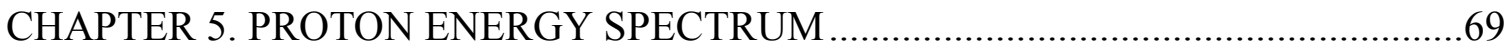

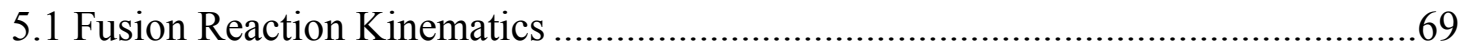

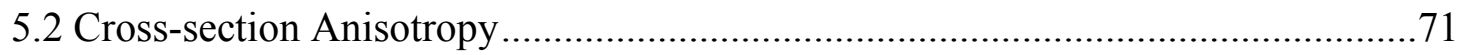

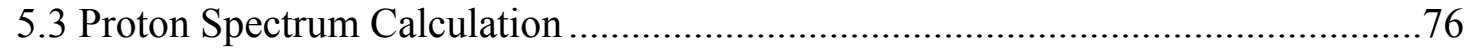

5.4 Pitch Angle Variation Along the Probing Trajectory …………………..................85

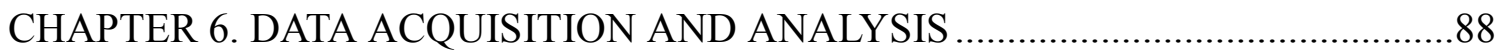

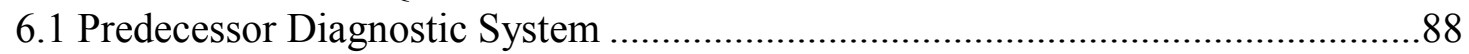

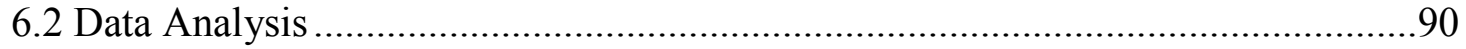

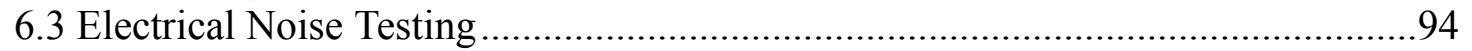

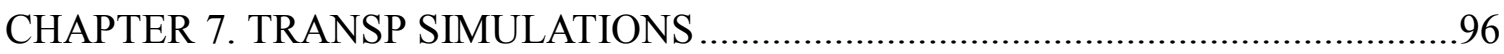

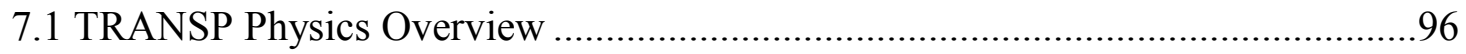

7.2 Application of the Kick Model [25]................................................................99

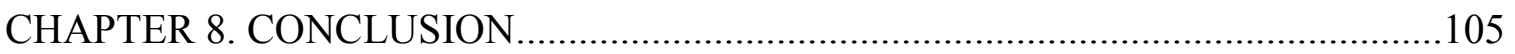

LIST OF REFERENCES ...............................................................................108

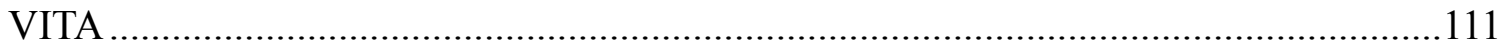


1. U.S. energy consumption by source $(1 \mathrm{BTU}=0.293 \mathrm{Wh})[1] \ldots \ldots \ldots \ldots \ldots \ldots \ldots \ldots \ldots \ldots \ldots \ldots . . .1$

2. Nuclear binding energy per nucleon [2] .......................................................... 3

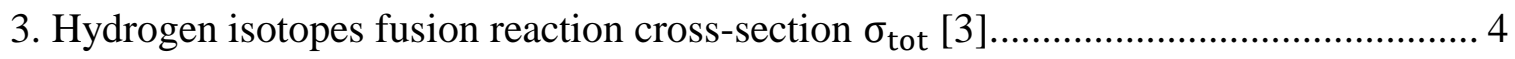

4. Plasma reactivity based on parametrization by H.-S. Bosch and G.M. Hale [3]........... 6

5. Tokamak types (conventional and spherical). ........................................................... 9

6. NSTX-U and MAST-U tokamaks schematic view [6], [7] ....................................... 10

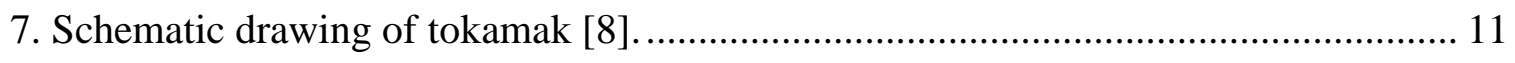

8. NSTX-U neutral beam injectors (NBI), top view [11] .......................................... 11

9. NSTX-U magnetic field lines shape [12]............................................................ 13

10. Neutral Beam Injection scheme [13] ................................................................... 14

11. Helical orbit of charged particle in magnetic field. ……………............................. 15

12. The bad a) and the good b) magnetic field curvature effect. ...................................... 18

13. MAST plasma poloidal cross section shape reference geometry in normalized

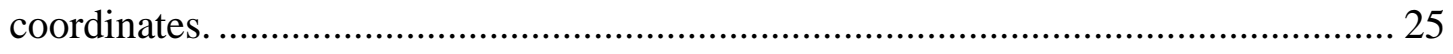

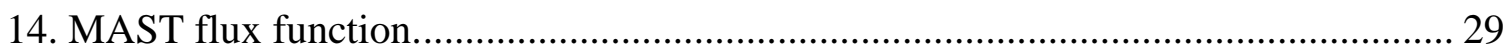

15. Magnetic field components magnitude in normalized coordinates............................ 31

15. Current density profiles in normalized coordinates.................................................. 30

17. Safety factor profile vs. start coordinate................................................................. 32

18. Safety factor profile as a function of flux value. …………………......................... 33

19. MAST-U EFIT calculated flux function in normalized coordinates. ......................... 34

20. Poloidal and toroidal current densities (MAST-U EFIT results) in normalized

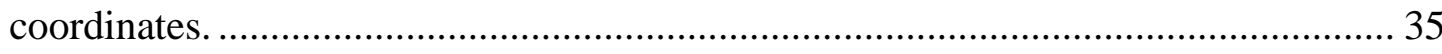


21. MAST-U magnetic field (EFIT reconstructed) in normalized coordinates................ 36

22. Safety factor $\mathrm{q}$ as a function of flux surface defined by flux value $\psi$....................... 36

23. Ion implanted silicon surface barrier detector. ....................................................... 38

24. Plasma probing central trajectories for NSTX-U [14] .............................................. 39

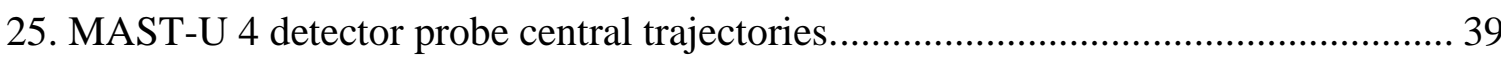

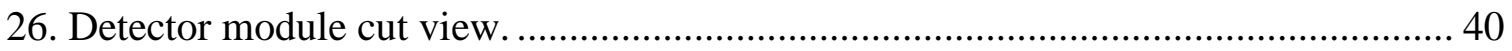

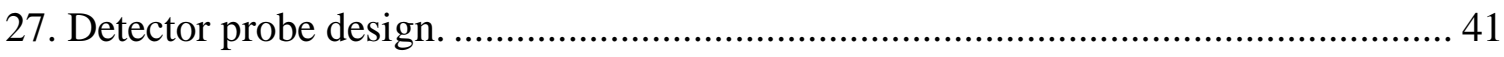

28. Machined 6-detector probe head (stainless steel SS316)........................................... 42

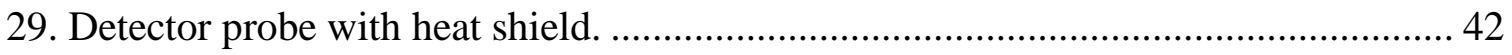

30. 8 detector probe (heatshield is not shown), with probing trajectories. ....................... 43

31. Reciprocating arm and support structure mounted on the diagnostic port outside of the vessel (requires adaptation for MAST-U). ....................................................... 46

32. Reciprocating arm retraction range (probe head shown in green) ............................ 47

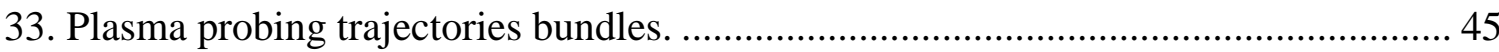

34. MAST-U 8 channel probing bundles with fusion emissivity profile shown as a

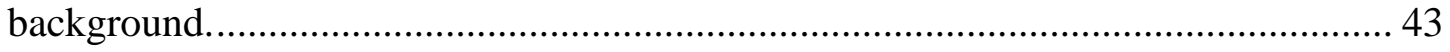

35. Probing trajectory bundles for varying radial head position (varying distance from

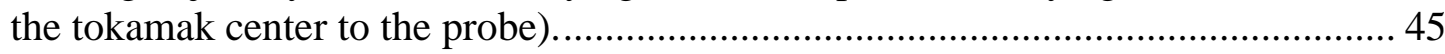

36. Probing trajectory bundles for varying axial head rotation. ....................................... 46

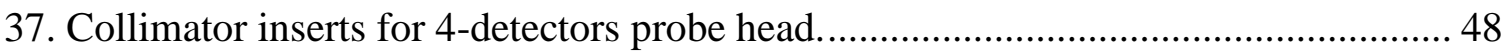

38. Collimator/detector schematic splitting for bundles calculations.............................. 49

39. Trajectory curvature on detector - collimator distance. ............................................. 51

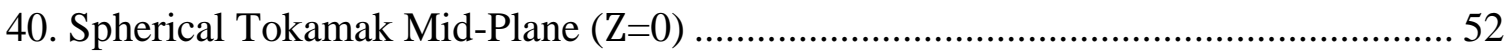

41. Midplane crossing shift due to trajectory direction correction angle $\alpha$ in a uniform

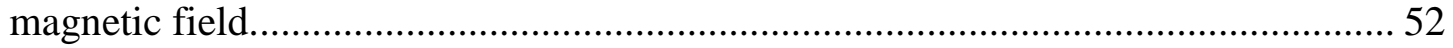


42. Trajectories bundles midplane crossing with and without correction.

43. Predicted rate change due to starting trajectory angle correction. ............................. 54

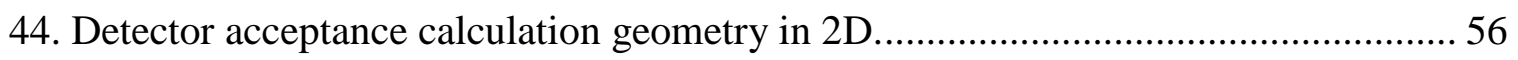

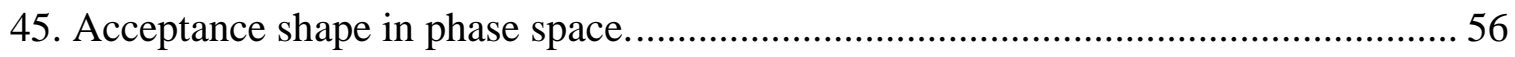

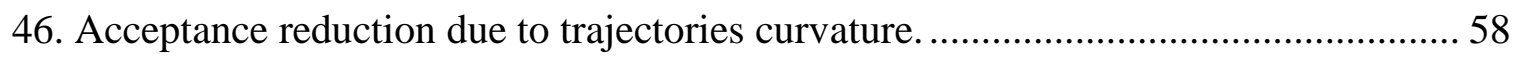

47. Monte Carlo acceptance calculation trajectories without magnetic field. ................. 59

48. Monte Carlo calculated acceptance (normalized by theoretical value) with relative

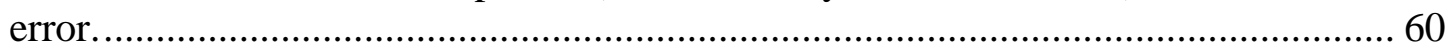

49. Monte Carlo acceptance calculation trajectories (2D) with magnetic field............... 61

50. Monte Carlo simulated detector acceptance phase space points (black dots), and theoretical acceptance without magnetic field (blue parallelogram).

51. Phase space manifold of accepted trajectories showing the time evolution of the detector phase-space acceptance ensemble.

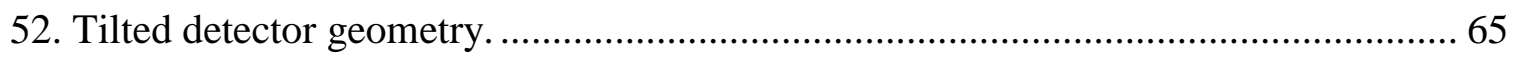

53. Reaction kinematics geometry (3D) depicting the fast beam ions (index 1) and fusion product (index 3) velocities unit vectors orientation relative to the magnetic field vector at the fusion reaction location.

54. Differential cross-section of $\mathrm{D}(\mathrm{d}, \mathrm{p}) \mathrm{T}$ fusion for incident energy of $69.9 \mathrm{KeV}$ as a function of center of mass angle [21]......

55. Differential cross-section in C.M. and laboratory frame. The differences are caused by transition from the moving C.M. to the laboratory frame.

56. Emissivity anisotropy scaling factor as a function of the proton pitch angle $\chi_{3}$ for different pitch angles $\chi_{1}$ of a monoenergetic $69.9 \mathrm{keV}$ deuteron beam.

57. Cross-section anisotropy effect on predicted rates.

58. Proton energy vs scattering angle $\left(\theta_{\mathrm{lab}}\right)$ in the laboratory frame for the $\mathrm{D}(\mathrm{d}, \mathrm{p}) \mathrm{T}$ reaction at $69.9 \mathrm{keV}$ beam energy. 
59. Proton spectrum from isotropic monoenergetic $(69.9 \mathrm{keV})$ beam fusion with target $(1 \mathrm{keV})$ plasma ions with isotropic cross-section (Monte Carlo simulation with 106 samples).

60. Proton spectrum from isotropic monoenergetic $(69.9 \mathrm{keV})$ beam fusion with target $(1 \mathrm{keV})$ plasma ions with anisotropic cross-section (Monte Carlo simulation with 106 samples)

61. Proton spectrum emitted perpendicular to magnetic field $\left(\chi_{3}=\pi / 2\right)$ from anisotropic $\left(\chi_{1}=\pi / 2\right)$ monoenergetic $(69.9 \mathrm{keV})$ beam fusion with target $(1 \mathrm{keV})$ plasma ions (isotropic cross-section assumed).

62. Proton spectrum emitted by isotropic monoenergetic $(69.9 \mathrm{keV})$ beam fusion with target $(1 \mathrm{keV})$ plasma ions with isotropic reaction cross-section (convolution of reduced distribution function).

63. Analytical and Monte Carlo simulated proton spectrum emitted at $\chi_{3}=\pi / 3$ pitch angle, from fusion with anisotropic deuteron beam of pitch angle $\chi_{1}=2 \pi / 3$ and energy distribution $\mathrm{f}(\mathrm{E}) \propto \exp \left(-1 / 2 \cdot(\mathrm{E}-69.9 \mathrm{keV})^{2} /(17 \mathrm{keV})^{2}\right)$.

64. Analytical and Monte Carlo simulated proton spectrum emitted at $\chi_{3}=\pi / 3$ pitch angle, from fusion with anisotropic deuteron beam of pitch angle $\chi_{1}=\pi / 2$ and energy distribution $\mathrm{f}(\mathrm{E}) \propto \exp \left(-1 / 2 \cdot(\mathrm{E}-69.9 \mathrm{keV})^{2} /(17 \mathrm{keV})^{2}\right)$.

65. Analytical and Monte Carlo simulated proton spectrum emitted at $\chi_{3}=\pi / 3$ pitch angle, deuteron beam pitch angle $\chi_{1}=\pi / 3$ and energy distribution $\mathrm{f}(\mathrm{E}) \propto$ $\exp \left(-1 / 2 \cdot(\mathrm{E}-69.9 \mathrm{keV})^{2} /(17 \mathrm{keV})^{2}\right)$

66. Analytical and Monte Carlo simulated proton spectrum emitted at $\chi_{3}=\pi / 3$ pitch angle, from fusion with anisotropic deuteron beam of pitch angle $\chi_{1}=\pi / 6$ and energy distribution $\mathrm{f}(\mathrm{E}) \propto \exp \left(-1 / 2 \cdot(\mathrm{E}-69.9 \mathrm{keV})^{2} /(17 \mathrm{keV})^{2}\right)$.

67. Pitch angle of the central probing trajectory as a function of radial and vertical position along the central probing trajectory for 4 detectors.

68. Expected proton spectrum emitted from thermal ions fusion with anisotropic deuteron beam of pitch angle $\chi_{1}$ and energy distribution $\mathrm{f}(\mathrm{E}) \propto \exp (-1 / 2$. $\left.(\mathrm{E}-69.9 \mathrm{keV})^{2} /(17 \mathrm{keV})^{2}\right)$ detected by the channel with given pitch angle $\chi_{3}$ variation along the central probing trajectory.

69. Data acquisition electronics scheme [19].

70. Typical signal shape from MAST data. The proton peak amplitude is $\sim 0.7 \mathrm{~V}$ and the triton peak is $\sim 0.23 \mathrm{~V}$ ( $3 \mathrm{MeV}$ and $1 \mathrm{MeV}$ energy particles), the peak width is of the order of $100 \mathrm{~ns}$. 
71. Superposition of all selected normalized peak data (points) and the common normalized peak shape.

72. Subset of fitted data. A 10th order polynomial has been used as to model the background and the signal baseline.

73. Experimental rates for MAST 2013 shot 29975 (1 ms integration). Data for three channels (three detectors) are shown with the shaded area around each curve being a statistical uncertainty.

74. NSTX-U electrical noise testing results

75. Passing (left) and trapped (right) fast ion gyrocenter motion for a poloidal crosssectional projection. Dashed lines represent the equilibrium flux surfaces

76. MAST 2013 experimental campaign Charged Fusion Products Diagnostic (CFPD) count rates comparison with TRANSP emissivity calculated rates [24]. Scenario 1 is a quiescent MHD plasma discharge, and Scenario 6 is a sawtooth instability plasma discharge.

77. Plasma discharge (shot) 29976 emissivity profile where the Kick model was applied to the fast ion transport simulation in TRANSP. Upper right plot is the emissivity as a function of the radial coordinate for a fixed vertical position (horizontal slice at $\mathrm{z}=25.6 \mathrm{~cm}$ ). The bottom right plot shows a slice of the emissivity at constant $r=100.8 \mathrm{~cm}$ as function of $\mathrm{z}$. The red cross indicates the intersection point of the two slices (vertical and horizontal).

78. Shot 29976 emissivity profile where the Kick model was applied with doubled kick amplitudes scaling to the fast ion transport simulation in TRANSP. Upper right plot is the emissivity as a function of the radial coordinate for a fixed vertical position (horizontal slice at $\mathrm{z}=25.6 \mathrm{~cm}$ ). The bottom right plot shows a slice of the emissivity at constant $r=100.8 \mathrm{~cm}$ as function of $\mathrm{z}$. The red cross indicates the intersection point of the two slices (vertical and horizontal). 102

79. MAST shot 29976 measured and predicted rates with kick model TRANSP simulated emissivity profiles. Measured rates data is not available for Channel 0 (Detector 1) and Channel 1 (Detector 2) after $0.19 \mathrm{~s}$ presumably due to the neutral beam spray particle overload and of bad quality for Channel 3 (Detector 4) because of partial obstruction of the sightline.

80. MAST shot 29976 Channel 2 measured proton rate and rate predicted with TRANSP simulated emissivity profile with a kick model applied and kick amplitude scaling factor set to 1 and 2 . 


\section{CHAPTER 1. INTRODUCTION}

Major attempts to solve humanity's growing energy demands together with the efforts to eliminate the ecological impact of energy production, such as carbon dioxide emission and radioactive waste, has led the scientific community to the conclusion that controlled thermo-nuclear fusion could be one of the main candidates to address those issues.

\section{U.S. Energy Consumption}

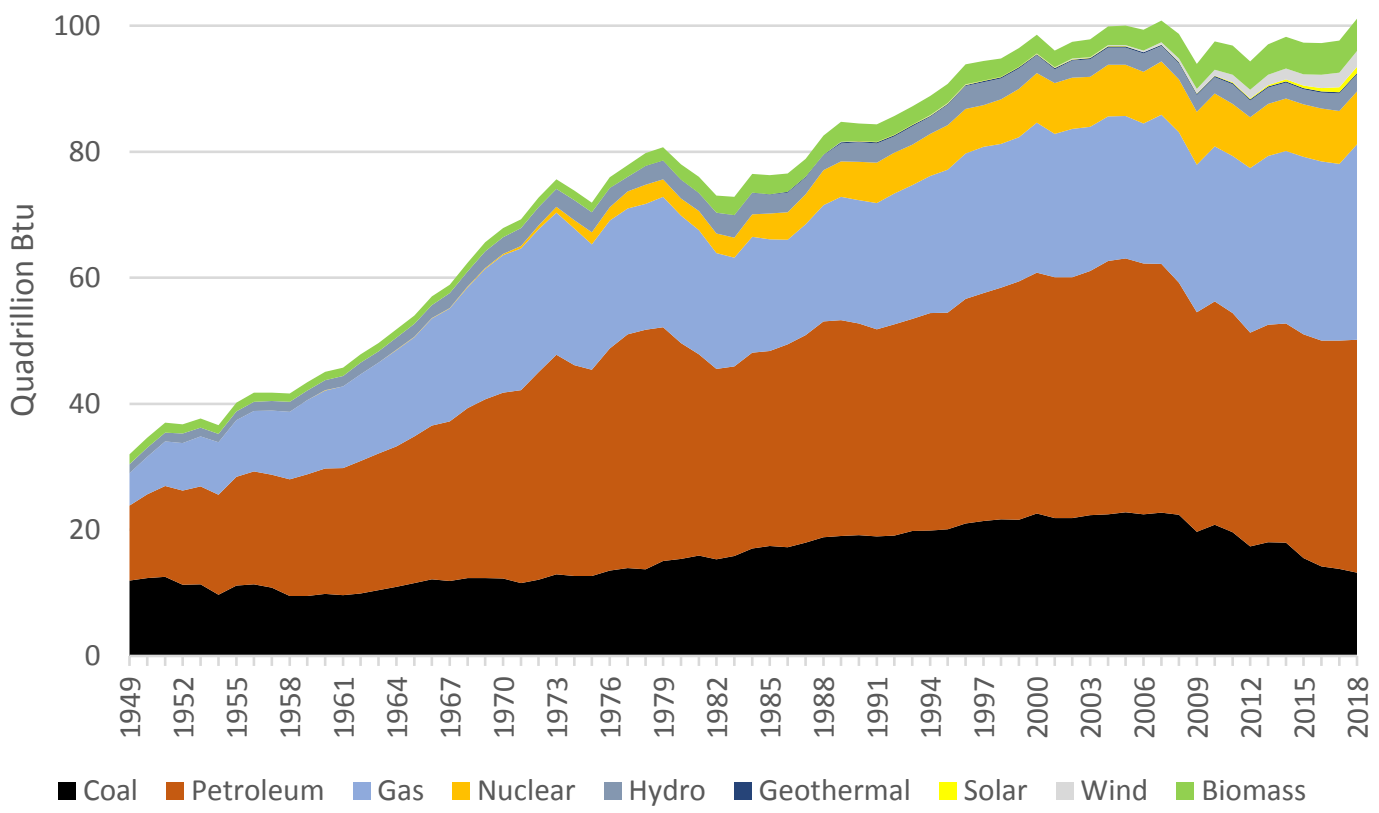

Figure 1. U.S. energy consumption by source (1 BTU $=0.293 \mathrm{Wh})$ [1].

The source structure of the U.S. energy consumption, shown in Figure 1, is taken from U.S. Energy Information Administration July 2019 Monthly Energy Review. It clearly indicates that the contribution percentage of clean energy sources remains extremely low. Total energy consumption itself is constantly growing noticeably, showing more than a threefold increase in the past 70 years. The growth can be explained by population growth and an increase of energy demand per capita due to living standards improvements. 
Thermonuclear fusion as a prospective energy source possesses several advantages compared to existing energy production technologies. Zero $\mathrm{CO}_{2}$ emission is a main benefit in contrast to the fossil fuels burning, practical absence of long-lived nuclear waste and intrinsically high reactor safety with respect to potential accidents is a crucial factor in comparison to fission nuclear reactors and incredibly high energy capacity compared to renewable and other energy sources.

In nuclear fusion, energy is released when two light nuclei combine into a heavier one and additional light reaction products and when the product's total mass is smaller than the sum of the initial nuclei masses. The mass difference $\Delta m=\left(m_{1}+m_{2}\right)-\left(m_{3}+m_{4}\right)$, where $m_{1}, m_{2}$ are initial and $m_{3}, m_{4}$ are resulting nuclei masses, is converted into kinetic energy of the reaction products according to well-known relation $Q=\Delta m c^{2}$. The $Q$ value in nuclear science denotes the amount of energy released or absorbed during a nuclear reaction.

The total number of nucleons is conserved during the nuclear reaction, but the mass difference comes from the binding energy per nucleon, which varies with the number of nucleons in a nucleus (Figure 2). The binding energy combines the contribution of a longrange Coulomb repulsion force and a short-range nuclear attraction force acting on the nucleons. For lighter nuclei the binding energy per nucleon is higher, since when the nucleus is small and each nucleon is mostly within the strong interaction range of the rest of nucleons, the Coulomb repulsion is then well compensated by the strong attraction. In larger nuclei there are more nucleons that are out of range of strong interaction of each other, but they still repel through electro-magnetic interaction, which makes it less bound. 


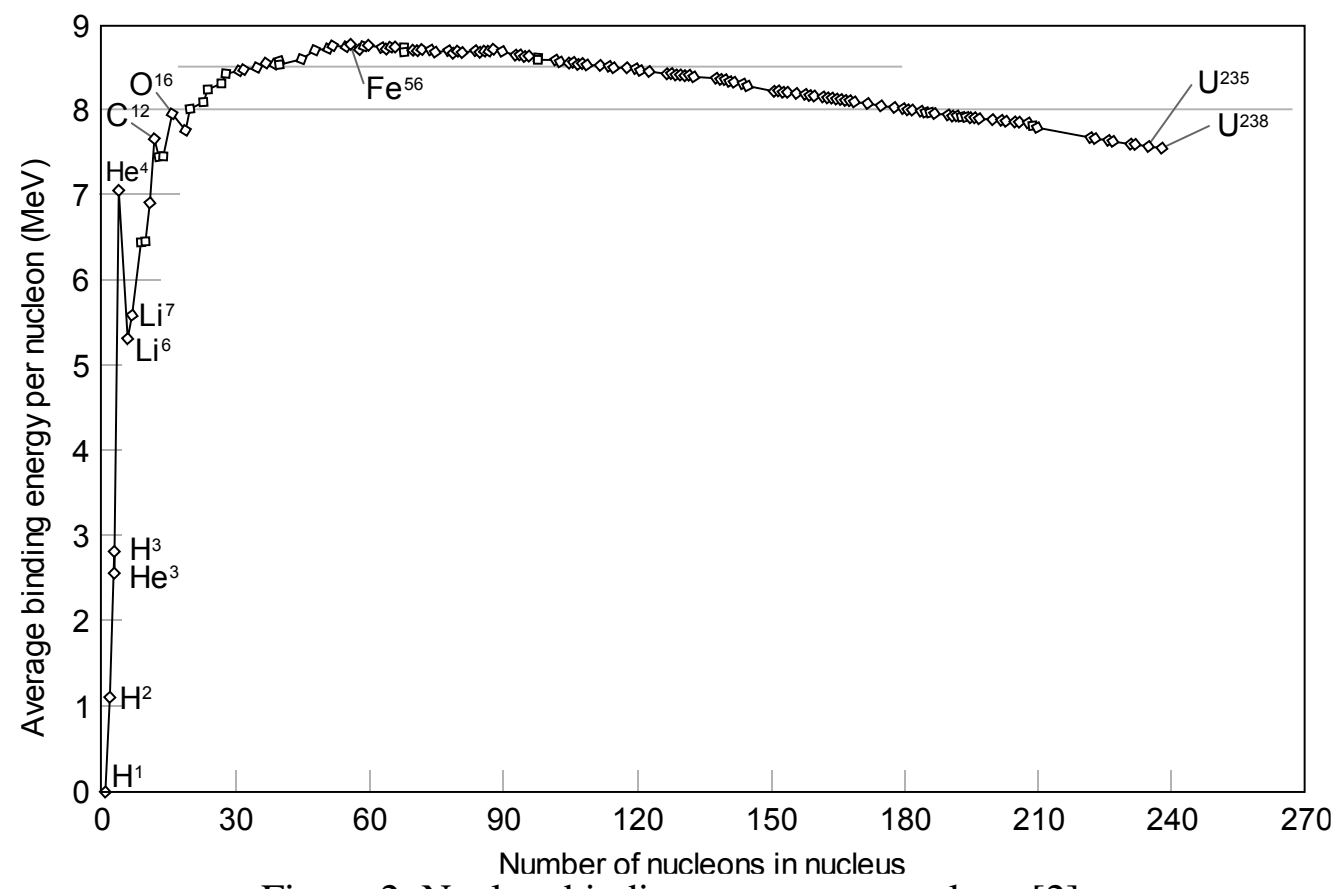

Figure 2. Nuclear binding energy per nucleon [2].

The isotope ${ }^{56} \mathrm{Fe}$ has the highest binding energy per nucleon, or equivalently the smallest mass per nucleon, meaning that all the fusion reactions with the products up to the ${ }^{56} \mathrm{Fe}$ isotope can occur with release of energy. Of course, since Coulomb repulsion is proportional to the reacting nuclei charges, a higher kinetic energy of the colliding nuclei would be necessary to overcome the potential barrier, thus the lighter isotopes are much more favorable. In practice, fusion reactions between hydrogen isotopes are of the most interest for energy production.

$$
\begin{aligned}
& { }^{2} \mathrm{H}+{ }^{2} \mathrm{H} \rightarrow{ }^{1} \mathrm{H}+{ }^{3} \mathrm{H}+4.03 \mathrm{MeV} \\
& { }^{2} \mathrm{H}+{ }^{2} \mathrm{H} \rightarrow{ }^{3} \mathrm{He}+n+3.27 \mathrm{MeV} \\
& { }^{2} \mathrm{H}+{ }^{3} \mathrm{H} \rightarrow{ }^{4} \mathrm{He}+n+18.4 \mathrm{MeV}
\end{aligned}
$$

By comparison, the energy released in burning one $\mathrm{CH}_{4}$ molecule is of the order of $10 \mathrm{eV}$, six orders of magnitude less than the energy released in a fusion reaction. 
The abundance of deuterium $\mathrm{D}\left({ }^{2} \mathrm{H}\right)$ in sea water makes it a perfect hydrogen isotope for the fusion research in facilities such as tokamaks or stellarators. Deuterium by itself is not the best option for the future energy generating fusion reactors since the D-D $\left({ }^{2} \mathrm{H}+{ }^{2} \mathrm{H}\right)$ reaction cross-section is much lower than the $\mathrm{D}-\mathrm{T}\left({ }^{2} \mathrm{H}+{ }^{3} \mathrm{H}\right)$ cross-section by several orders of magnitude as can be seen in Figure 3 below. The plot was produced with the crosssection parametrization published by H.-S. Bosch and G.M. Hale [3].

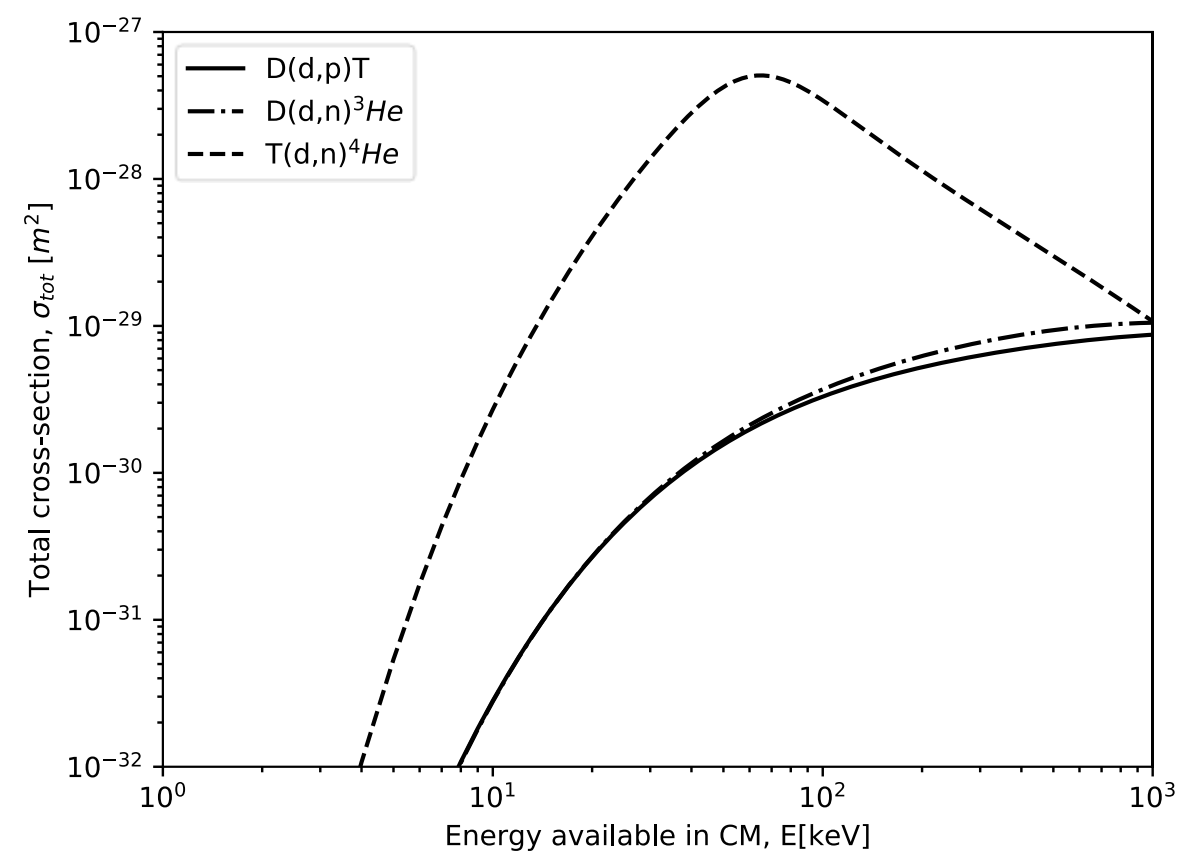

Figure 3. Hydrogen isotopes fusion reaction cross-section $\sigma_{t o t}[3]$.

Both D-D reaction branches given in Equation 1 and Equation 2 have similar crosssection values, which makes them practically equally probable when deuterons fuse, but for the accurate fusion rate calculations the branching ratio should be considered since cross-sections for $p$ and $n$ branches can differ by up to $15 \%$. As can be seen from Figure 3, the D-T reaction cross-section has a maximum around $70 \mathrm{keV}$, which corresponds to the temperature of roughly $0.81 \times 10^{9} \mathrm{~K}$. It is much higher than the sun's core temperature, 
which is around $15 \times 10^{6} \mathrm{~K}$. The huge pressure and density of the sun's core maintained by the gravitational pull compensates for the smaller cross-section at that temperature, keeping the reactivity high enough to maintain the burning process. In magnetically confined plasmas that value of pressure is not achievable since it would require unfeasible magnetic field magnitudes, hence operating at increased temperatures is the only option.

\subsection{Plasma Reactivity and Ignition Condition}

The reactivity of a plasma is defined by the following expression:

$$
\langle\sigma v\rangle=\iint f_{1}\left(\vec{v}_{1}\right) f_{2}\left(\vec{v}_{2}\right)\left|\vec{v}_{1}-\vec{v}_{2}\right| \sigma\left(\left|\vec{v}_{1}-\vec{v}_{2}\right|\right) d \vec{v}_{1} d \vec{v}_{2}
$$

where $\vec{v}_{1}, \vec{v}_{2}$ are the plasma ion species velocity vectors, $\sigma$ is a reaction differential crosssection (which can be angle dependent in a general case) and $f(\vec{v})$ is a normalized velocity distribution function of a given species at a given location. The reaction rate per unit volume per unit time, often called emissivity, then can be found as:

$$
\xi=\frac{n_{1} n_{2}}{1+\delta_{1,2}}\langle\sigma v\rangle
$$

where $n_{1}, n_{2}$ are the species densities at a given location, and $\delta$ is a Kronecker's delta to prevent double counting for particles of the same species reactions.

For plasmas with a Maxwellian distribution function of the ion velocities, the reactivity has the temperature dependence shown in Figure 4. The reactivity has a maximum at a temperature of about $70 \mathrm{keV}$, which is much higher than typical ion temperatures in future tokamaks, such as ITER (International Thermonuclear Experimental Reactor). Nevertheless, at a temperature of about $15 \mathrm{keV}$ the reactivity is high enough to be able to 
reach the break even, $Q=1$, condition and even higher values of $Q$. The $Q$ factor is a ratio between the power generated by fusion reactions inside the plasma and the input power supplied for plasma heating from external sources such as ohmic heating, neutral beam injection, and radio frequency $(\mathrm{RF})$ heating.

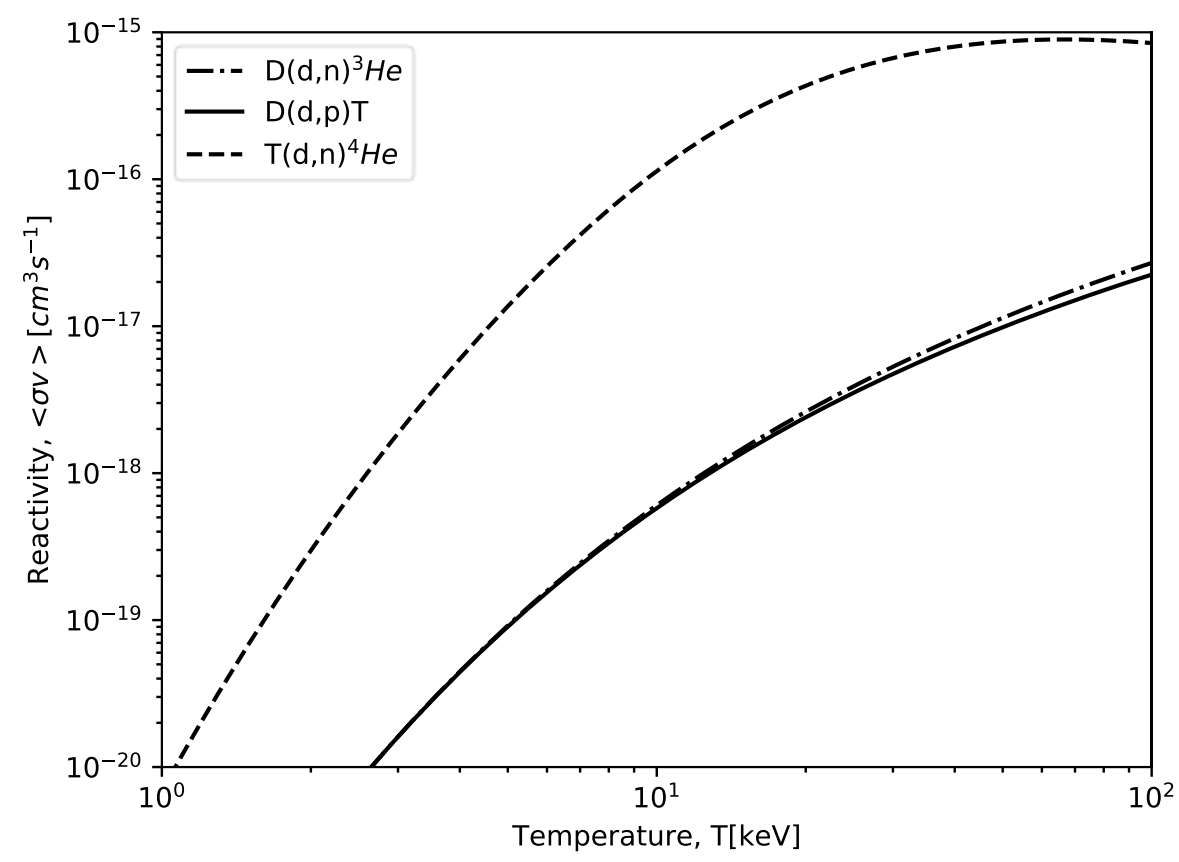

Figure 4. Plasma reactivity based on parametrization by H.-S. Bosch and G.M. Hale [3].

Another critical plasma property is the ignition condition that depends on the energy confinement time $\tau_{E}$, which characterizes how quickly the plasma loses all energy stored in it. When the fusion product alpha particles can fully compensate the energy loss rate: $P_{\text {loss }}=E_{\text {stored }} / \tau_{e}$ and sustain the plasma burning process without the external energy input then ignition happens. Stored energy per unit volume for the Maxwellian plasma can be found as: 


$$
E_{\text {thermal }}=\frac{3 n k T}{2},
$$

where $n$ is a total plasma density $\left(n=2 n_{e}\right.$ for electrically neutral plasma, where $n_{e}$ is electron density), $k$ is a Boltzmann constant, $T$ is a plasma temperature.

The ignition criterion, also referred to as a Lawson criterion, for a deuterium tritium plasma with equal isotopes densities $\left(n_{d}=n_{t}=n_{e} / 2\right)$ can then be written as

$$
\frac{3 n_{e} k T}{\tau_{E}} \leq \frac{n_{e}^{2}}{4}\langle\sigma v\rangle E_{\alpha}
$$

where $E_{\alpha}$ is the single alpha particle energy produced in a fusion reaction. The right side of the inequality in Equation 7 is the energy deposited by the alpha particles in the plasma, which should be greater or equal to the stored energy loss rate. The ignition criterium can be rewritten as follows:

$$
n_{e} \tau_{E} \geq \frac{12 k T}{\langle\sigma v\rangle E_{\alpha}} .
$$

For the ITER planned scenario of the steady state $D-T$ plasma at $25 \mathrm{keV}$ temperature this leads to $n \tau_{E}$ estimate of $1.5 \times 10^{20} \mathrm{~m}^{-3} \mathrm{~s}$ being necessary for plasma ignition.

Although the tritium being a rare isotope with a half-life of only about 12 years, it can be produced using the lithium neutron activation reaction since most of the released energy is carried by neutrons and fusion reactors produce a significant neutron flux. For the ITER reactor a special breeder blanket is being developed containing lithium bearing ceramics such as $\mathrm{Li}_{2} \mathrm{TiO}_{3}$ and $\mathrm{Li}_{4} \mathrm{SiO}_{4}$. 


\subsection{Fusion Devices}

There are two main approaches to achieve controlled nuclear fusion, one depends on inertial plasma confinement and the other is magnetic confinement fusion. Both methods are being studied experimentally and theoretically, and facilities are being developed and operated with preference given to the magnetic confinement devices.

Currently the most promising magnetic plasma confinement concepts can be divided into two main categories: tokamaks and stellarators, both having toroidal geometry but different magnetic field configurations. Compared to the inertial confinement method, which conceptually remains a tiny hydrogen bomb where the role of the outer nuclear fission shell is played by the intense laser pulses that hit the fuel pellet and squeeze and heat it up to ignite the reaction which then lasts until the plasma ball quickly disintegrates after a few nanoseconds, plasma pulses in magnetic confinement machines can last from few seconds up to several minutes as proposed for ITER.

The tokamak, which is currently the prevailing magnetic confinement device, was proposed by Soviet scientists in the mid 50s and gained a large recognition by the fusion community in the late $60 \mathrm{~s}$. The acronym TOKAMAK - a special kind of abbreviation for the Russian TOroidalnaya KAmera s MAgnitnimy Katushkami, which means Toroidal Chamber with Magnetic Coils. As the name implies, a tokamak uses a toroidally shaped magnetic field configuration to confine a plasma in the shape of a doughnut.

Currently there are two main types of tokamaks called the conventional tokamaks and the spherical tokamaks. The main difference of these two types is the aspect ratio of the plasma shape. The aspect ratio, $A$, is defined as a ratio between the major radius, $R_{0}$, of the 
plasma torus and the minor radius, $a$, of the plasma poloidal cross-section (Figure 5). Tokamaks with an aspect ratio larger than 3 are normally called conventional, and with $A \leq 2$ considered as spherical. The elongation parameter $k$ is a measure of the vertical stretch of the plasma poloidal cross-section profile $(k=b / a)$.

Spherical tokamaks have some advantages over conventional ones. One is reduced cost because of the economical magnetic coils, since it is easier to achieve the desired level of magnetic field with a smaller size of the plasma major radius. Another advantage is improved plasma stability because of the plasma cross-section geometry and magnetic field line shape which allows particles to stay longer on the inner side of the torus where it is inherently more stable because of the good curvature of magnetic field lines (explained elsewhere in the chapter).

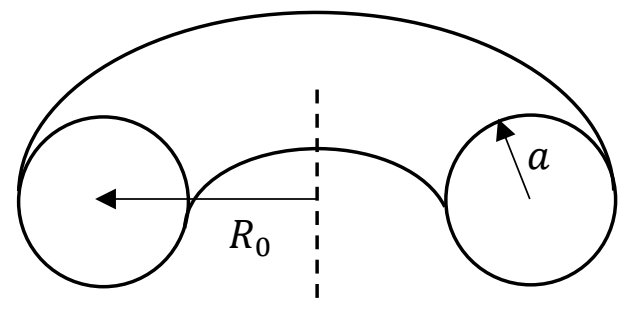

Standard tokamak, $A \geq 3$

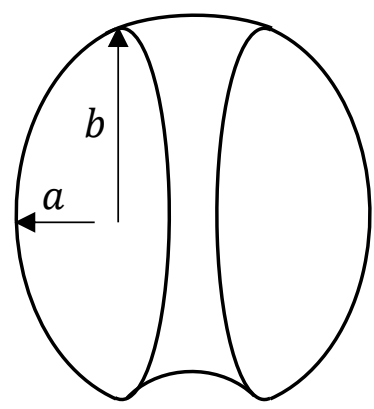

Spherical tokamak, $A \leq 2$

Figure 5. Tokamak types (conventional and spherical).

The charged fusion product diagnostic described in the thesis is especially well suited for spherical tokamaks such as the NSTX-U (National Spherical Torus Experiment Upgrade) at the PPPL (Princeton Plasma Physics Laboratory) and the MAST-U (Mega Amp Spherical Tokamak Upgrade) at the CCFE (Culham Centre for Fusion Energy), as shown in Figure 6. 


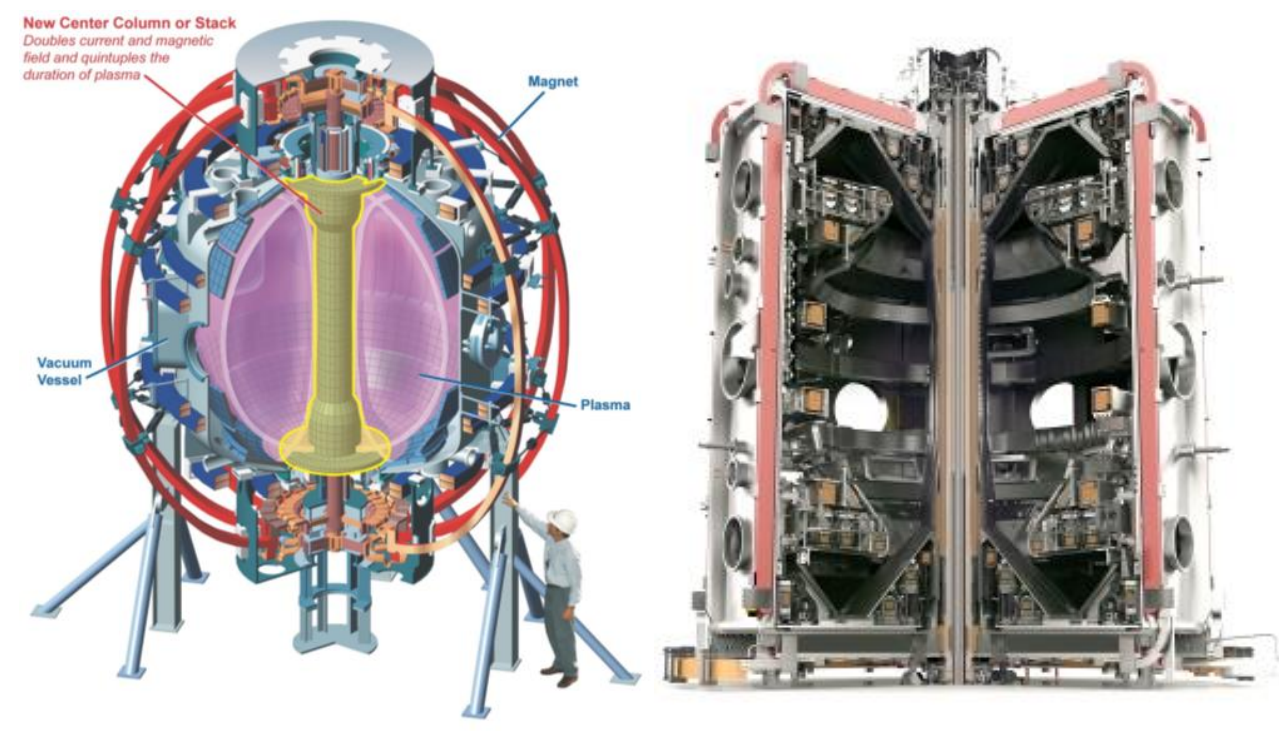

Figure 6. NSTX-U and MAST-U tokamaks schematic view [6], [7].

A plasma is created by ionization the deuterium gas inside the chamber by the induced toroidal electric field. The field is the result of a time varying vertical magnetic field flux created by the varying (ramping) current in a central column solenoid of the tokamak (Figure 7). Thus, the central solenoid is serving as a primary coil and a plasma/gas being a secondary coil as per transformer principle.

Ohmic heating can be efficient up to the point when the plasma becomes highly conductive. The plasma resistivity according to Spitzer [9] has a temperature dependence as $\eta \sim T^{-\frac{3}{2}}$, and the ohmic heating power density deposition in plasma is $P_{\Omega}=\eta j^{2}$, where $j$ is a current density. The current density has its limitation; to avoid plasma disruption the average current density should be such that the safety factor $q$ (which is defined in the next chapter) at the edge of the plasma be more the 2 , and the current density should not be too high in general to prevent the MHD (Magneto Hydro Dynamic) instabilities in the central region of the plasma, in particular the sawtooth instability [10]. Another constraint the 
ohmic heating has is the maximum current that can be driven through the center stack solenoid coils, meaning that the current ramping should be stopped at some point and the induced electric potential in the plasma will vanish.

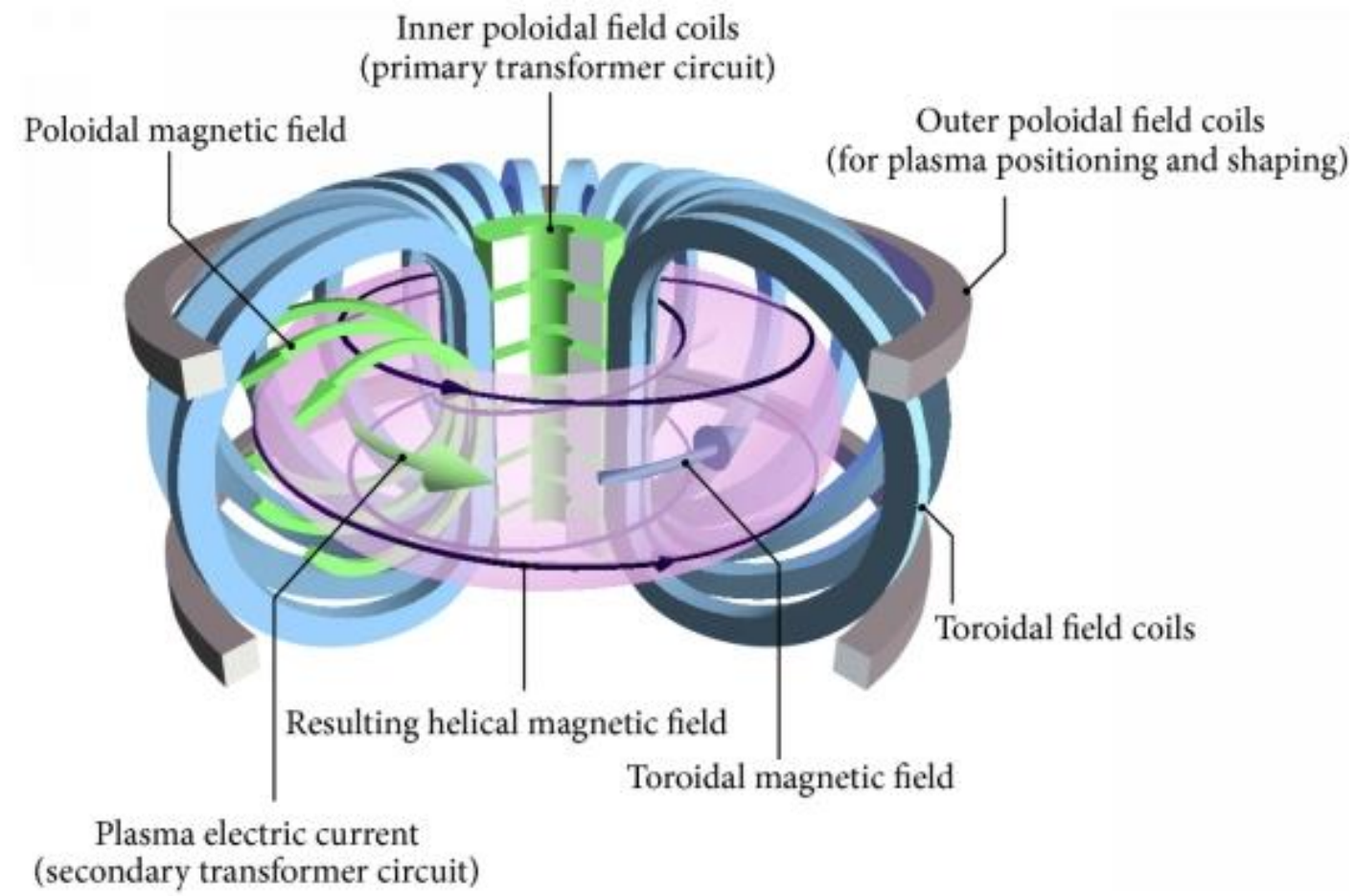

Figure 7. Schematic drawing of tokamak [8].

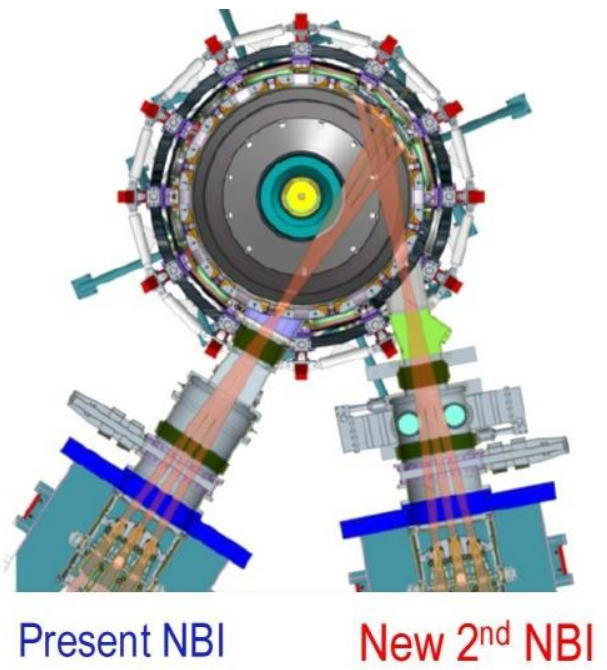

Figure 8. NSTX-U neutral beam injectors (NBI), top view [11]. 
Additional to the ohmic heating, there are other methods to deliver the energy to the plasma such as RF waves and neutral beam injection (Figure 8). The last one plays a key role in our research and will be described in more detail.

\subsection{MAST-U Tokamak}

The main parameters of the MAST-U are listed in the Table 1 . The major radius $R_{m}$ is the radius of the torus measured from the center of the doughnut hole to the center of the plasma, the aspect ratio $A$ is the ratio between the major radius and the effective radius of the confined plasma poloidal cross-sectional area, $A=R_{m} / r_{e f f}$, where $r_{e f f}$ can be defined as $r_{e f f}=2 \mathrm{~V} / \mathrm{S}, \mathrm{V}$ being a volume enclosed by the last closed flux surface with surface area $S$. The aspect ratio of a standard tokamak is about 3 , and the closer this ratio gets to 1 the plasma shape becomes more spherical. $I_{p}$ is the electric current flowing in the plasma in the toroidal direction (plasma current). $B_{t}$ is the toroidal component of the magnetic field at the magnetic axis (the point of maximum or minimum of magnetic flux). The pulse length $t_{p u l s e}$ is the duration of the plasma pulse. $P_{N B I}$ is the power injected into the plasma by a beam of neutral particles with an energy $E_{i n j}$, and $n_{e}$ is the electron density in the plasma.

As can be noted from Table 1, MAST-U has the aspect ratio $A=1.4$, consequently it belongs to the spherical tokamaks category. The magnetic field for plasma confinement in spherical tokamaks has a complex structure that must conform to the equilibrium conditions, which will be discussed in more details in the next chapter. In stellarators it is achieved with a set of intricately shaped coils, while in tokamaks it is created by combination of the external toroidal field coils, external poloidal field coils, and an 
additional poloidal component produced by the plasma current. A typical shape of the magnetic field line for a spherical tokamak depicting its intricacy is shown in Figure 9.

\begin{tabular}{|l|c|c|}
\hline \multicolumn{1}{|c|}{ Parameter } & MAST-U $1^{\text {st }}$ Campaign & Design Value \\
\hline$R_{m}[\mathrm{~m}]$ & 0.7 & 0.7 \\
\hline$A$ & 1.4 & 1.4 \\
\hline$I_{p}[\mathrm{MA}]$ & 1.0 & 2.0 \\
\hline$B_{t}[\mathrm{~T}]$ & 0.65 & 0.8 \\
\hline$t_{\text {pulse }}[\mathrm{s}]$ & 2 & 5 \\
\hline$P_{N B I}[\mathrm{MW}]$ & 3.5 & 5.0 \\
\hline$E_{\text {inj }}[\mathrm{keV}]$ & 75 & 75 \\
\hline$n_{e}\left[\mathrm{~m}^{-3}\right]$ & $\sim 1 \times 10^{-20}$ & $\sim 1 \times 10^{-20}$ \\
\hline
\end{tabular}

Table 1. MAST-U main parameters.

Plasma stability in tokamaks requires very high plasma currents (in the range of mega ampere) in order to create the appropriate magnetic field for plasma equilibrium. Since the inductive current drive through the ohmic heating has its limitations, neutral beam injection is used to achieve high plasma temperatures and to increase and drive the plasma current for better confinement stability.

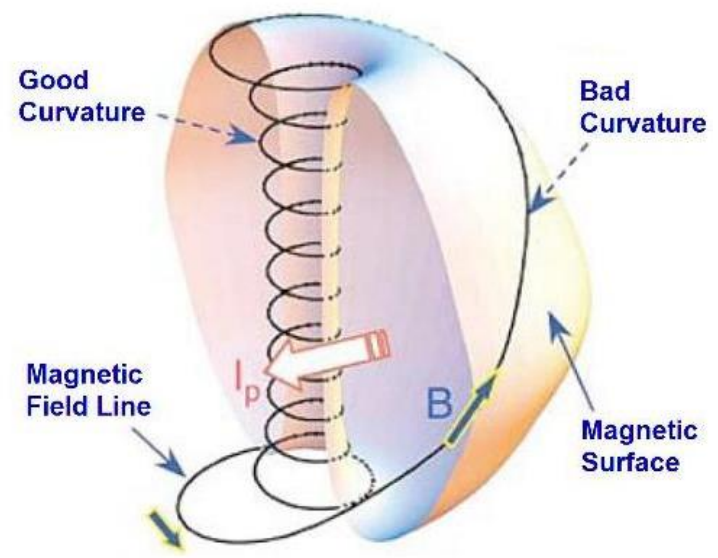

Figure 9. NSTX-U magnetic field lines shape [12]. 
The neutral beam is produced in the following steps: first deuterium gas is ionized in the ion source and the ions are then accelerated by the electrostatic field toward the accelerating grid. After passing through the neutralizing gas where a charge-exchange reaction happens between beam ions and gas due to collisional electron detachment, the accelerated particles pass through the deflection magnet to remove remaining charged particles, resulting in a neutral beam of energetic particles (Figure 10).

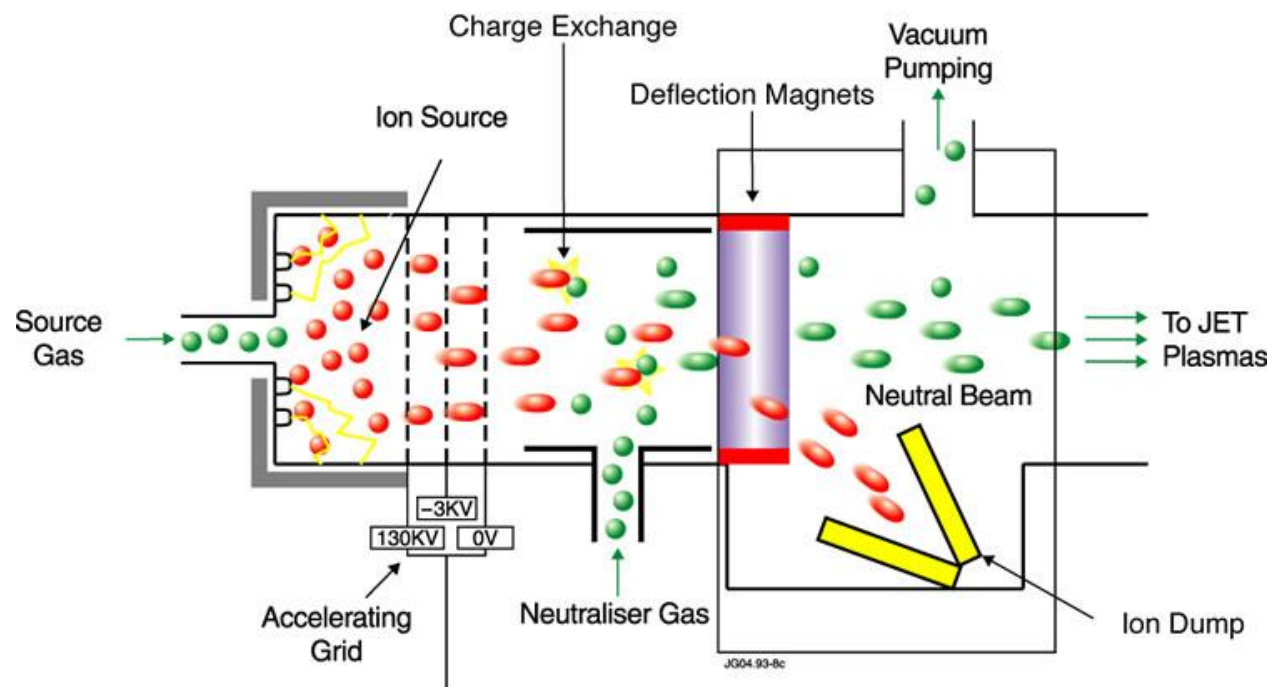

Figure 10. Neutral Beam Injection scheme [13].

It is interesting to note that while particle energy is relatively low compared to high energy accelerator beams, the power carried by the beam is high, meaning that the beam current (before neutralization, or the neutral atoms flow) has to be large. For $3.5 \mathrm{MW}$ of beam power and a $75 \mathrm{keV}$ beam energy, the current is $I_{i n j}=P_{i n j} / E_{\text {inj }} \approx 46 \mathrm{~A}$, compared to typical accelerator currents in the $\mu \mathrm{A}$ to $\mathrm{mA}$ range. Because of the high energy of the beam particles $(\sim 75 \mathrm{keV})$, compared to the thermal energy of the plasma ions, which is typically a few $\mathrm{keV}$, the cross-section for a nuclear reaction between beam-plasma particles and beam-beam particles is orders of magnitude higher than for thermal-thermal plasma 
particle interaction. As a consequence, the dominant portion of the fusion reactions in NSTX-U or MAST-U occur when neutral beam injection is on.

\subsection{Single Particle Motion and Drift Velocities}

The advantage of spherical tokamaks mentioned earlier - an improved plasma stability because of the shape of the plasma - can be understood from the concept of the good and the bad magnetic field curvature shown in Figure 9. On the basis of single particle motion in a magnetic field it can be explained why the good curvature field-line surfaces are more stable for plasma confinement. In a uniform magnetic field charged particles move on a helical trajectory following a field line, with a radius of gyration defined by

$$
R_{\text {gyro }}=\frac{m v_{\perp}}{q B}
$$

where $m$ is the particle mass, $v_{\perp}$ is the velocity component perpendicular to magnetic field, $q$ is the particle charge, $B$ is the magnetic field magnitude (Figure 11).

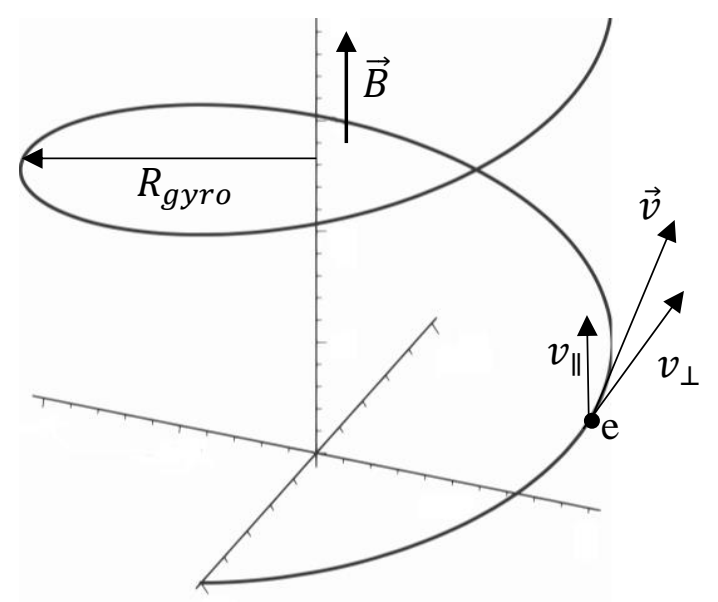

Figure 11. Helical orbit of charged particle in magnetic field. 
Ions and electrons of a typical MAST plasma have a temperature about $1 \mathrm{keV}$ and experience a magnetic field of about $0.65 \mathrm{~T}$ resulting in gyro radii of $10 \mathrm{~mm}$ and $0.16 \mathrm{~mm}$ respectively. Fusion products such as $3 \mathrm{MeV}$ protons or $1 \mathrm{MeV}$ tritons have a Larmor radius in this magnetic field of around $54 \mathrm{~cm}$, which is comparable with the size of the chamber itself. These particles are unconfined and quickly leave the plasma with a very small probability of colliding with plasma particles.

When the magnetic field has a gradient or a curvature, or if an external force is applied to a particle by an electric field for example, the particle will experience a so-called drift motion [4]. This is where its gyro center will no longer simply follow a field line but will drift with a certain velocity perpendicular to the field lines. The curvature drift is defined by

$$
\vec{v}_{c}=-\frac{m v_{\|}}{R q B^{2}}(\hat{\vec{k}} \times \vec{B}),
$$

where $R$ is the field curvature radius, $v_{\|}$is the velocity component parallel to the magnetic field, and $\hat{k}$ is the curvature direction unit vector, which can be expressed through the magnetic field unit vector $\hat{\vec{B}}$ and a curvature radius as

$$
\vec{k}=R(\hat{\vec{B}} \cdot \nabla) \hat{\vec{B}}
$$

Since $\hat{\vec{k}}$ is a unit vector one can find the curvature radius as

$$
R^{-1}=|(\hat{\vec{B}} \cdot \nabla) \hat{\vec{B}}|
$$

The drift velocity in a static electric field is given by the expression 


$$
\vec{v}_{E}=\frac{\vec{E} \times \vec{B}}{B^{2}}
$$

where $\vec{E}$ is the electric field vector [5].

\subsection{The Good and the Bad Magnetic Field Curvature}

When a plasma particle moves in the tokamak magnetic field on the plasma boundary surface, with its gyro center approximately following the field line, it passes the regions of the so-called good and the bad magnetic field curvature (Figure 9).

As can be noted from the Equation 10 the curvature drift velocity is charge dependent, which leads to the opposite drift of positive and negative particles. If the plasma surface is smooth this will not cause the electric charge density to deviate from neutrality. However, when the surface is disturbed with some ripple, which can result from interaction with the neutral beam e.g., the curvature drift causes the opposite charge densities to occur either side of the ripple humps (Figure 12). These oppositely charged regions produce an electric field and leads to the so-called $\vec{E} \times \vec{B}$ drift, which is charge independent (Equation 13). It can cause either dampening or a growth of the disturbance, depending on the relative orientation of the magnetic field curvature and the pressure gradient.

In case shown in Figure 12 a) the pressure gradient has the same direction as the field curvature vector $\vec{k}$, and the $\vec{E} \times \vec{B}$ drift effect strengthens the initial perturbance (worsening the ripple) and can cause a ballooning instability. It is said that the magnetic

field has a bad curvature in this case. In the second case (Figure 12 b) the $\vec{E} \times \vec{B}$ drift effect weakens the perturbance (flattening the ripple) and hence stabilizes the plasma surface. It 
can be summarized that when the magnetic-field line-curvature center is located inside the plasma (or in other words the curvature vector points into the plasma) it is considered as a bad curvature field, and vice versa. More aspects of the plasma stability will be discussed later.
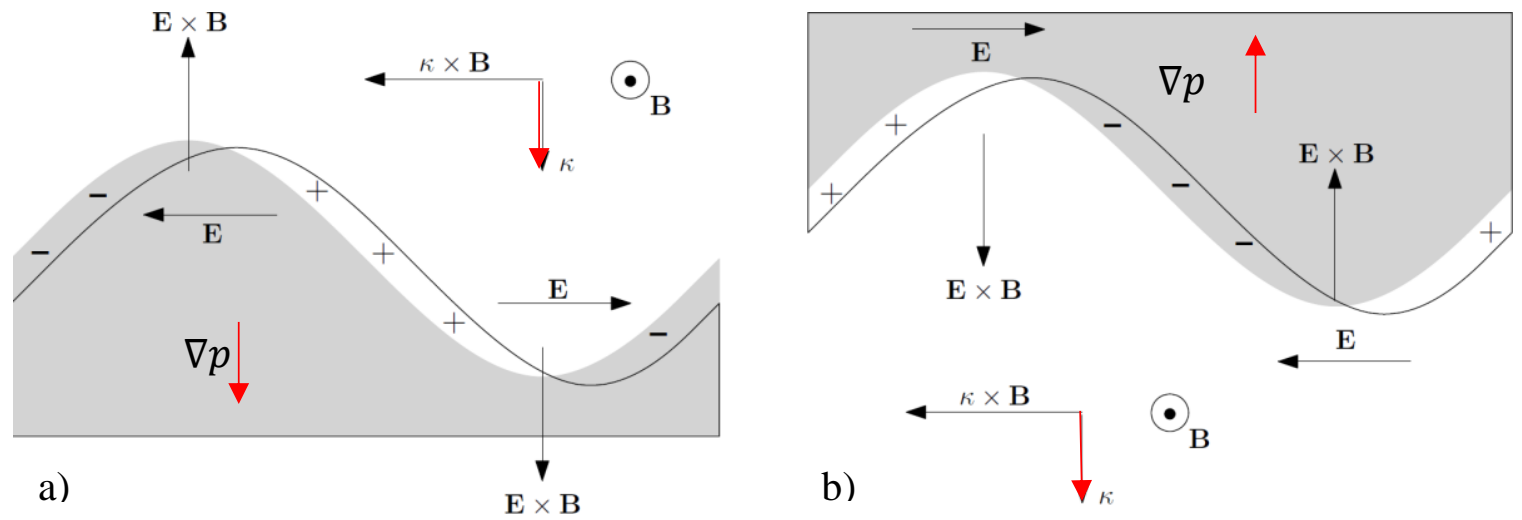

Figure 12. The bad a) and the good b) magnetic field curvature effect. 


\section{CHAPTER 2. PLASMA MAGNETO HYDRO DYNAMIC (MHD) EQUILIBRIUM}

Most of the formulas listed in Chapter 2 can be found in various plasma physics books that address the MHD description of plasma dynamics. The goal of the work done and described hereafter was to implement an analytical solution to plasma equilibrium reconstruction and to relate that solution to the existing tokamak parameters. This is typically not described in the literature.

The analytical approach to describe plasma dynamics on a macroscopic scale starts with the definition of the probability distribution function for plasma particles of species $\alpha$ which shows the particle probability density in the velocity domain and the particle number density at a given coordinate in space and at time $t$ such that

$$
\int f_{\alpha}(\vec{r}, \vec{v}, t) d^{3} v=n_{\alpha}(\vec{r}, t)
$$

The time evolution of the distribution function is governed by the Boltzmann equation:

$$
\frac{\partial f_{\alpha}}{\partial t}+\vec{v} \cdot \nabla_{r} f_{\alpha}+\vec{a} \cdot \nabla_{v} f_{\alpha}=\left(\frac{\delta f_{\alpha}}{\delta t}\right)_{c o l l}
$$

where $\vec{a}$ is a particle acceleration cause by the electromagnetic force acting on it a given location at time $t$ :

$$
\vec{a}(\vec{r}, t)=\frac{q_{\alpha}}{m_{\alpha}}[\vec{E}(\vec{r}, t)+\vec{v} \times \vec{B}(\vec{r}, t)]
$$

where $q_{\alpha}$ is the particle charge, and $m_{\alpha}$ is the particle mass.

\subsection{Single Fluid Force Balance Equation}

Multiplying Equation 15 by velocity $\vec{v}$ and integrating over the velocity space one can arrive at a single-species force balance equation. Considering the plasma consisting of one 
type of ion and electrons, and combining the force balance equation for these two particle species, the force balance equation in a single fluid approach can be obtained [14]:

$$
\rho_{\mathrm{m}}\left[\frac{\partial}{\partial \mathrm{t}}+\overrightarrow{\mathrm{u}} \cdot \nabla\right] \overrightarrow{\mathrm{u}}=-\nabla \cdot \overline{\mathrm{P}}+\rho \overrightarrow{\mathrm{E}}+\overrightarrow{\mathrm{J}} \times \overrightarrow{\mathrm{B}}
$$

where $\rho_{\mathrm{m}}$ is the mass density, $\overrightarrow{\mathrm{u}}$ is the local fluid velocity, $\overline{\mathrm{P}}$ is the pressure tensor, $\rho$ is the charge density, $\vec{J}$ is the current density and $\vec{B}$ is the magnetic field vector.

To simplify Equation 17 one can assume that the plasma is in static equilibrium, hence the left side of the equation is equal to zero, the pressure can be taken as a scalar quantity, and the charge density is zero assuming that the plasma is quasi-neutral, which leads to:

$$
\nabla p=\overrightarrow{\mathrm{J}} \times \overrightarrow{\mathrm{B}}
$$

Equation 18 shows that the pressure gradient in plasma should be balanced by the Lorentz force acting on the local current density for the plasma to be in equilibrium.

The so-called stream function can be defined as follows,

$$
\psi(r, z)=r A_{\phi}(r, z)
$$

where $\vec{A}$ is the vector potential satisfying the relation

$$
\nabla \times \vec{B}=\vec{A}
$$

The magnetic field is then related to the vector potential $\vec{A}$ in the azimuthally symmetric case where there is no dependence on the toroidal coordinate $\phi$ as follows:

$$
B_{r}=\frac{\partial A_{\phi}}{\partial z} ; \quad B_{\phi}=\frac{\partial A_{r}}{\partial z}-\frac{\partial A_{z}}{\partial r} ; \quad B_{z}=\frac{1}{r} \frac{\partial\left(r A_{\phi}\right)}{\partial r}
$$

Then $\vec{B}$ can be expressed through the stream function as

$$
B_{r}=-\frac{1}{r} \frac{\partial \psi}{\partial z} ; \quad B_{z}=\frac{1}{r} \frac{\partial \psi}{\partial r}
$$

Since the pressure gradient is perpendicular to the magnetic field, then 


$$
\frac{\partial p}{\partial r} \frac{\partial \psi}{\partial z}-\frac{\partial p}{\partial z} \frac{\partial \psi}{\partial r}=0
$$

Equation 23 implies that $\nabla p \times \nabla \psi=0$, thus the pressure can be treated as a function of the stream function, i.e. $p=p(\psi)$.

From the fact that the pressure gradient is perpendicular to the current density, it is possible to show that the product of the poloidal field and the radius $r$ can be treated as a function of $\psi$

$$
r B_{\phi}=F(\psi)
$$

More details can be found in Jeffrey Friedberg Plasma Physics and Fusion Energy [16].

\subsection{Grad-Shafranov Equation and Solov'ev Profiles}

Using the above assertions, the force balance Equation 18, and Maxwell's equations:

$$
\nabla \cdot \vec{B}=0 ; \quad \nabla \times \vec{B}=\mu_{0} \vec{J}
$$

one can arrive at the Grad-Shafranov equation. This is a nonlinear partial differential equation of elliptical form:

$$
\mu_{0} r^{2} \frac{d p}{d \psi}+F \frac{d F}{d \psi}+\left(\Delta^{*} \psi\right)=0
$$

where $\Delta^{*}$ is the Stokes operator defined as

$$
\Delta^{*} \psi=r^{2} \nabla \cdot\left(\frac{1}{r^{2}} \nabla \psi\right)
$$

$\psi(r, z)$ is commonly referred to as a flux function since it can be shown that the stream function is proportional to the poloidal magnetic field flux through the toroidal circular contour passing through the $(r, z)$ coordinate. 
Indeed

$$
F_{c t o r}(r, z)=\int_{0}^{r} B_{z} 2 \pi r d r=\int_{0}^{r} \frac{1}{r} \frac{\partial \psi}{\partial r} 2 \pi r d r=2 \pi \psi(r, z)
$$

where $F_{c t o r}$ is the poloidal field flux through the circular toroidal contour that passes thorough the point $(r, z)$ in the poloidal cross section.

The Grad-Shafranov equation can be solved analytically or numerically only if one explicitly specifies the pressure and poloidal field dependence on the flux function $\psi$. One relatively simple example of the equilibrium solution can be obtained using the Solov'ev proposed profiles for $p$ and $F$ [15], which are defined by the following equations,

$$
\begin{gathered}
\mu_{0} \frac{d p}{d \psi}=-C ; \quad F \frac{d F}{d \psi}=-A \\
p=-\frac{C}{\mu_{0}} \psi ; \quad F=\sqrt{2\left(K_{F}-A \psi\right)},
\end{gathered}
$$

where $A, C$ and $K_{F}$ are some constants that need to be determined.

If one scales the coordinate variables by the major radius $R$, and rearrange the constants,

$$
r=R x ; \quad z=R y ; \quad \psi=\psi_{0} \chi ; \quad \psi_{0}=R^{2}\left(A+C R^{2}\right),
$$

one can rewrite the Grad-Shafranov equation in a simpler form:

$$
x \frac{\partial}{\partial x}\left(\frac{1}{x} \frac{\partial \chi}{\partial x}\right)+\frac{\partial^{2} \chi}{\partial y^{2}}=\gamma+(1-\gamma) x^{2} ; \quad \gamma=\frac{A}{A+C R^{2}}
$$

To implement the solution of the Grad-Shafranov equation for the specific tokamak one should clearly understand what free parameters are present in Equation 32 and how they can be found from the real machine geometrical and measured physical parameters. As was mentioned earlier, there are three unknown constants $A, C$ and $K_{F}$ in the equation. 
Scaled variables defined in Equation 31 combine two parameters $-A$ and $C$ - into another pair $\psi_{0}$ and $\gamma$, and bring the Grad-Shafranov equation to a more convenient form.

Once $\psi_{0}$ and $\gamma$ are determined, the other constants $A$ and $C$ can be calculated.

$$
A=\frac{\gamma \Psi_{0}}{R^{2}} ; \quad C=\frac{\Psi_{0}}{R^{4}}(1-\gamma)
$$

The determination of $\psi_{0}$ and $\gamma$ will be described later in this section. $R$ is a given machine major radius and is a known parameter.

The remaining integration constant $K_{F}$ can be found from the expression for the toroidal magnetic field at the geometric center of the machine $(X=1 . Y=0)$ (Figure 13):

$$
B_{t}(1,0)=\frac{F(1,0)}{R}=\frac{\sqrt{2\left(K_{F}-A \psi_{0} \chi(1,0)\right)}}{R}
$$

and given the toroidal magnetic field value $B_{t}(1,0)=B_{0}$ one can find,

$$
K_{F}=\frac{1}{2}\left(B_{t}^{2} R^{2}+2 A \Psi_{0} \chi(1,0)\right) .
$$

After the solution $\chi(x, y)$ is found, which itself depends on $\gamma$, and $\psi_{0}$ and $\gamma$ determined based on the given machine parameters imposing certain constraints on the solution, the full information of MHD equilibrium for this tokamak is found and various physical quantities can be obtained.

The MAST equilibrium profile is symmetric with respect to the midplane, the general solution of Equation (32) will have the following form:

$$
\chi(x, y)=\frac{\gamma}{2} x^{2} \ln x+\frac{1-\gamma}{8} x^{4}+\sum_{0}^{6} a_{j} \chi_{j}(x, y)
$$


where the homogeneous solution has been truncated to seven polynomials allowing us to satisfy seven geometry constraints as stated below. The explicit form of these polynomials is as follows:

$$
\begin{aligned}
& \chi_{0}^{e}(x, y)=1 \\
& \chi_{1}^{e}(x, y)=x^{2} \\
& \chi_{2}^{e}(x, y)=x^{2} \ln (x)-y^{2} \\
& \chi_{3}^{e}(x, y)=x^{4}-4 x^{2} y^{2} \\
& \chi_{4}^{e}(x, y)=\left(x^{4}-4 x^{2} y^{2}\right) \ln (x)-3 x^{2} y^{2}+\frac{2}{3} y^{4} \\
& \chi_{5}^{e}(x, y)=x^{6}-12 x^{4} y^{2}+8 x^{2} y^{4} \\
& \chi_{6}^{e}(x, y)=\left(x^{6}-12 x^{4} y^{2}+8 x^{2} y^{4}\right) \ln (x)-5 x^{4} y^{2}+\frac{28}{3} x^{2} y^{4}-\frac{8}{15} y^{6} .
\end{aligned}
$$

The upper index $\chi_{i}^{e}$ denotes that the polynomials are of even power in the $y$ coordinate. For vertically asymmetric machines, like NSTX or ITER, one would have to include odd power polynomials as well, but the overall procedure would stay the same. Note that $\gamma$ in this approach remains a parameter until the very last steps.

Geometry constraints are imposed on the reference surface (its cross section is shown in Figure 13) defined using the following parametric relation:

$$
X=1+\varepsilon \cos \left(\tau+\delta_{0} \sin \tau\right) ; \quad Y=\varepsilon k \sin \tau,
$$

where $\varepsilon=a / R$ is the tokamak inverse aspect ratio (a - minor radius), $k$ - elongation, and $\delta$ is a triangularity $\left(\delta_{0}=\arcsin \delta\right)$, three dimensionless parameters that define the geometry, $\tau$ is a running parameter in the range from 0 to $2 \pi$. The reference surface sets the desired equilibrium plasma shape. When the triangularity parameter $\delta$ is equal to 0 the 
plasma poloidal cross-section has an elliptical or circular shape (depending on the elongation), the closer $\delta$ gets to 1 , the more triangular the plasma cross-section shape becomes. The reference contour curvature at three selected points (Figure 13) can be found from the parametric Equation 44 as follows:

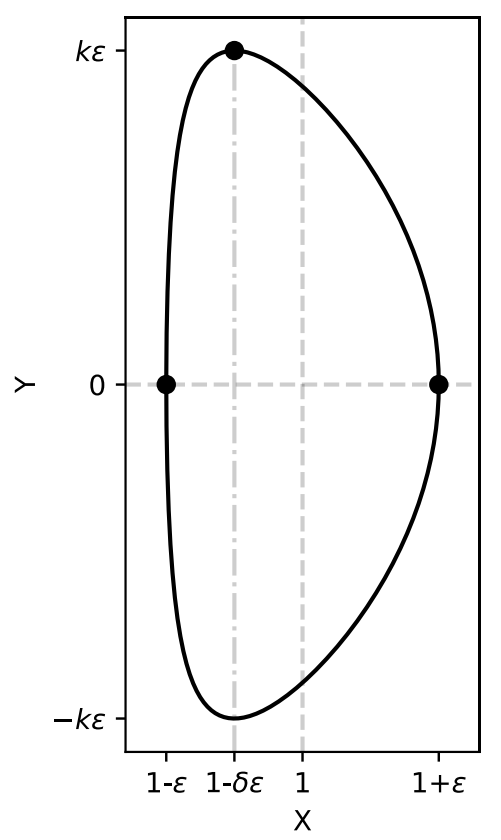

Figure 13. MAST plasma poloidal cross section shape reference geometry in normalized coordinates.

$$
\begin{aligned}
& N_{1}=\left[\frac{d^{2} x}{d y^{2}}\right]_{\tau=0}=-\frac{\left(1+\delta_{0}\right)^{2}}{\varepsilon k^{2}}, \\
& N_{2}=\left[\frac{d^{2} x}{d y^{2}}\right]_{\tau=\pi}=\frac{\left(1-\delta_{0}\right)^{2}}{\varepsilon k^{2}}, \\
& N_{3}=\left[\frac{d^{2} y}{d x^{2}}\right]_{\tau=\frac{\pi}{2}}=-\frac{k}{\varepsilon \cos ^{2} \delta_{0}} .
\end{aligned}
$$

The set of the equations representing the geometry constraints imposed on our solution function $\chi$ is listed below: 
1. $\chi(1+\varepsilon, 0)=0$,

2. $\chi_{y y}(1+\varepsilon, 0)=-N_{1} \chi_{x}(1+\varepsilon, 0)$,

3. $\chi(1-\varepsilon, 0)=0$,

4. $\chi_{y y}(1-\varepsilon, 0)=-N_{2} \chi_{x}(1-\varepsilon, 0)$,

5. $\chi(1-\delta \varepsilon, k \varepsilon)=0$,

6. $\chi_{x}(1-\delta \varepsilon, k \varepsilon)=0$,

7. $\chi_{x x}(1-\delta \varepsilon, k \varepsilon)=-N_{3} \chi_{y}(1-\delta \varepsilon, k \varepsilon)$. (outer point flux) (48)

(outer point curvature) (49)

(inner point flux) (50)

(inner point curvature) (51)

(high point flux) (52)

(high point slope) (53)

(high point curvature) (54)

Constraints 1,3,5,6 define the value of $\chi$ and its $x$ derivative at the high point to be zero. Conditions $2,4,7$ can be derived starting from the fact that $\chi$ should stay constant on the reference contour. The change of $\chi$ to first order is $d \chi=\chi_{x} d x+\chi_{y} d y$. For the inner and outer points, where $\frac{d x}{d y}=0, d \chi$ should be equal to 0 , thus $\chi_{y}$ should be equal to 0 as well. It can also be noted that when moving along the contour of constant $\chi$, the direction of the infinitesimal displacement should be perpendicular to the $\chi$ gradient at that point, or in other words $(d x, d y) \cdot\left(\chi_{x}, \chi_{y}\right)=0$.

Writing the change of $\chi$ to second order, assuming $x$ is a function of $y$, which is valid for inner and outer points and opposite for the upper point of reference curve, gives

$$
\begin{aligned}
& \mathrm{d} \chi=\chi_{x} \mathrm{dx}+\chi_{y} \mathrm{dy}+\frac{1}{2} \chi_{x x} \mathrm{~d} x^{2}+\chi_{x y} \mathrm{~d} x \mathrm{~d}+\frac{1}{2} \chi_{y y} \mathrm{~d} y^{2} \\
& =\chi_{x}\left(\frac{d x}{d y} d y+\frac{1}{2} \frac{d^{2} x}{d y^{2}} d y^{2}\right)+\chi_{y} \mathrm{~d} y+\frac{1}{2} \chi_{x x}\left(\frac{d x}{d y} d y\right)^{2}+\chi_{x y} \frac{d x}{d y} \mathrm{~d} y^{2}+\frac{1}{2} \chi_{y y} \mathrm{~d} y^{2} \\
& =\chi_{x} \frac{d x}{d y} \mathrm{dy}+\frac{1}{2} \chi_{x} \frac{d^{2} x}{d y^{2}} \mathrm{~d} y^{2}+\chi_{y} \mathrm{dy}+\frac{1}{2} \chi_{x x}\left(\frac{d x}{d y}\right)^{2} \mathrm{~d} y^{2}+\chi_{x y} \frac{d x}{d y} \mathrm{~d} y^{2}+\frac{1}{2} \chi_{y y} \mathrm{~d} y^{2} .
\end{aligned}
$$


Using the fact that $\frac{d x}{d y}=0$ and $\chi_{y}=0$ for constraints 1,4 , the first, third, fourth and fifth terms are equal to zero. Then the only term left is

$$
\frac{1}{2} \chi_{x} \frac{d^{2} x}{d y^{2}} d y^{2}+\frac{1}{2} \chi_{y y} d y^{2}=0 ; \quad \chi_{y y}=-N_{1,2} \chi_{x}
$$

Constrain number 7 can be proven in the similar manner.

Combining all constraints and using the explicit form of $\chi(x, y)$ from Equation 36 one obtains a set of equations that can be written in a matrix form:

$$
\begin{array}{lll}
A_{i j} a_{j}=\gamma B_{i}+C_{i} & \\
A_{1, j}=\chi^{j}(x, y) ; & B_{1}=\frac{1}{2} x^{2} \ln x-\frac{1}{8} x^{4} ; & C_{1}=\frac{1}{8} x^{4} ;\left.\right|_{(1+\varepsilon, 0)} \\
A_{2, j}=\chi_{y y}^{j}(x, y)+N_{1} \chi_{x}^{j}(x, y) ; & B_{2}=-N_{1}\left(x \ln x+\frac{1}{2} x-\frac{1}{2} x^{3}\right) ; & C_{2}=\frac{1}{2} x^{3} ;\left.\right|_{(1+\varepsilon, 0)} \\
A_{3, j}=\chi^{j}(x, y) ; & B_{3}=\frac{1}{2} x^{2} \ln x-\frac{1}{8} x^{4} ; & C_{3}=\frac{1}{8} x^{4} ;\left.\right|_{(1-\varepsilon, 0)} \\
\vdots & \vdots & \vdots
\end{array}
$$

Coefficients $a_{j}$ then can be found as functions of $\gamma$ by numerically inverting matrix $\mathrm{A}$ and

$$
a(\gamma)=\gamma A^{-1} B+A^{-1} C
$$

Since now the solution of the scaled Grad-Shafranov equation is known with one remaining parameter $\gamma$, one can numerically evaluate following integrals:

$$
\begin{gathered}
K_{1}=\int d x d y \frac{\gamma+(1-\gamma) x^{2}}{x}, \\
K_{2}=-\int x \chi(x, y) d x d y, \\
K_{3}=\int x d x d y .
\end{gathered}
$$

As a result, one will have

$$
K_{1}=\gamma K_{1}^{\gamma}+K_{1}^{0} ; \quad K_{2}=\gamma K_{2}^{\gamma}+K_{2}^{0} ; \quad K_{3}=K_{3}^{0}
$$


These integrals appear in machine safety factor equation $q_{*}$ and $\beta_{t}$ (ratio of plasma kinetic pressure to toroidal magnetic field pressure) as follows:

$$
q_{*}=\frac{\pi a^{2}\left(1+\kappa^{2}\right) B_{t}}{\psi_{0} K_{1}}=\frac{\pi \epsilon^{2}\left(1+\kappa^{2}\right) B_{t}}{\left(A+C R^{2}\right) K_{1}},
$$

and

$$
\beta_{t}=\frac{8 \pi^{2} \epsilon^{4}(1-\gamma)}{q_{*}{ }^{2}}\left(\frac{1+\kappa^{2}}{2}\right)^{2} \frac{K_{2}}{K_{1}^{2} K_{3}} .
$$

Combining these two equations one gets

$$
\begin{aligned}
\frac{1}{q_{*}} & =\frac{\psi_{0}}{\pi a^{2}\left(1+k^{2}\right) B_{0}}\left(\gamma K_{1}^{\gamma}+K_{1}^{0}\right), \\
\beta_{t} & =\frac{2 \varepsilon^{4}(1-\gamma) \psi_{0}^{2}}{a^{4} B_{0}^{2}} \frac{\left(\gamma K_{2}^{\gamma}+K_{2}^{0}\right)}{K_{3}} .
\end{aligned}
$$

Since know $\beta_{t}$ and $q^{*}$ are known for the specific machine, one can find $\gamma$ and $\psi_{0}$ from these two equations.

$$
\begin{array}{lr}
R=0.85 & \text { major radius in meters } \\
a=0.65 & \text { minor radius } \\
R & \\
\frac{a}{a}=1.3 & \text { aspec ratio } \\
k=2.45 & \text { elongation } \\
\delta=0.5 & \text { triangularity } \\
\beta_{t}=0.14 & \text { toroidal beta } \\
q^{*}=3.35 & \text { kink safety factor } \\
B_{0}=0.52 & \text { toroidal magnetic field at } \mathrm{R}
\end{array}
$$

Table 2. MAST parameters.

Once all free MAST specific constants have been determined using the MAST parameters (Table 2), one can find pressure, magnetic field, or current density at any point of the poloidal cross-section. Figure 14 shows the flux function $\psi$, the solution of 
Grad-Shafranov equation meeting the geometry constraints as well as matching the real machine physical parameters.

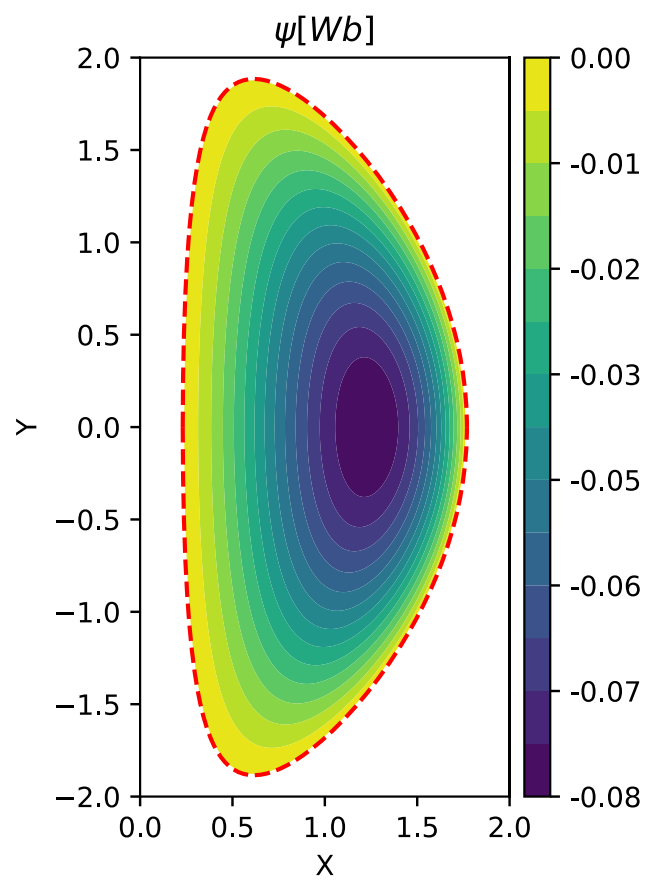

Figure 14. MAST flux function.

The current density profile can be found from

$$
\vec{J}=\frac{1}{\mu_{0} r} \frac{d F}{d \psi} \nabla \psi \times \widehat{\phi}+\left[r \frac{d p}{d \psi}+\frac{1}{\mu_{0} r} F \frac{d F}{d \psi}\right] \widehat{\phi} .
$$

Toroidal and poloidal current density profiles are shown in Figure 15. The magnetic field vector at an arbitrary point in the plasma is given by the following expression:

$$
\vec{B}=\frac{1}{r}[F \widehat{\phi}+\nabla \psi \times \widehat{\phi}]
$$

In Figure 16 the $\vec{B}$-field vector components are shown. The poloidal component of the magnetic field has two components, $B_{r}$ and $B_{z}$, the magnitude $B_{p o l}=\sqrt{B_{r}^{2}+B_{Z}^{2}}$ is shown and the direction of the vector can be easily imagined since it is always tangential to the 
flux surface. Note that poloidal magnetic field $\left(B_{p o l}\right)$ profile is almost identical to poloidal current density $\left(J_{p o l}\right)$ profile, which is because $J_{p o l}$ is related to $B_{p o l}$ through

$$
J_{p o l}=\frac{-A B_{p o l}}{\mu_{0} F}
$$

and $F$ in this case is changing very little inside the limiting geometry. In the general case, these two profiles can look quite different from each other.
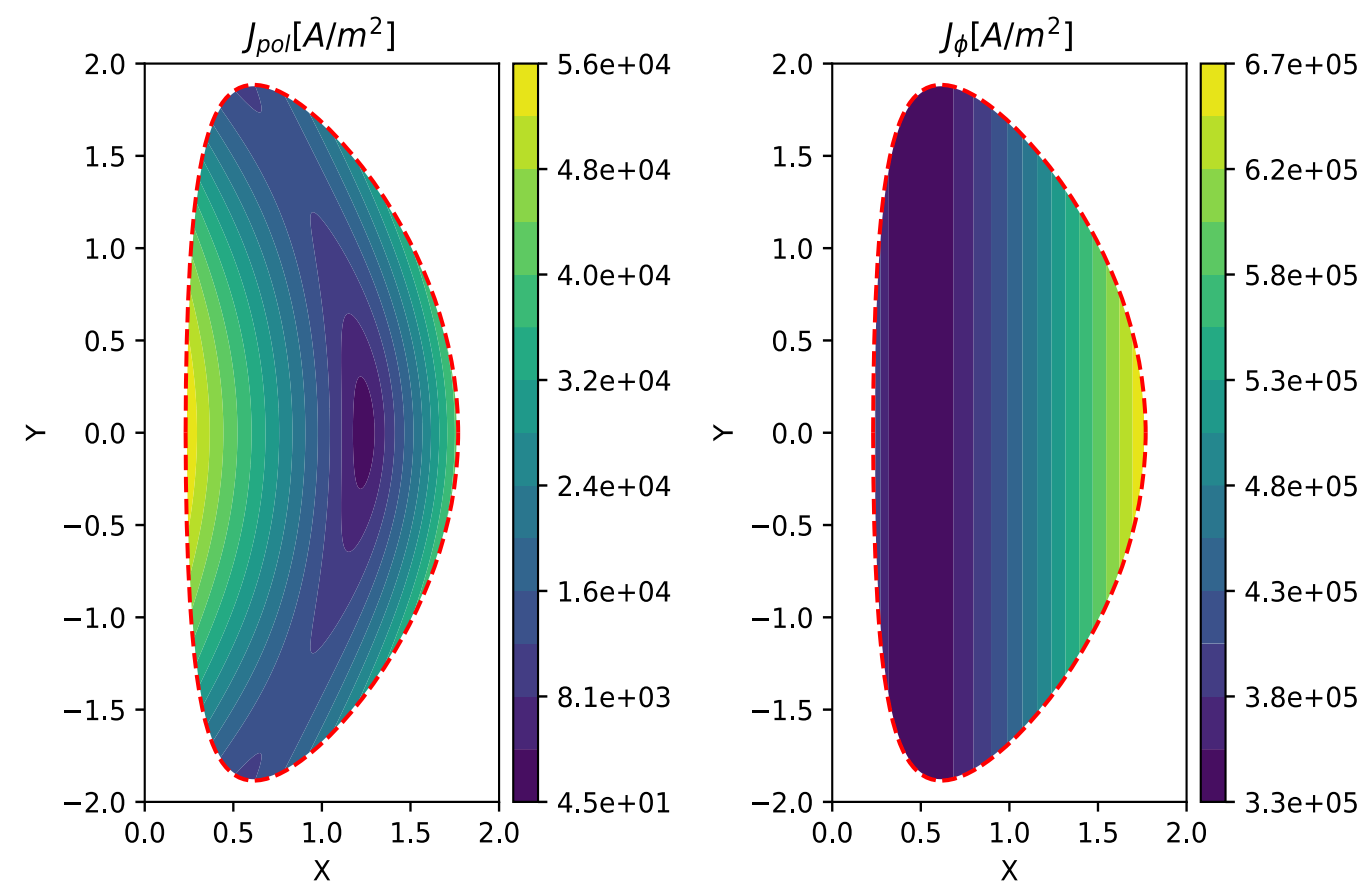

Figure 15. Current density profiles in normalized coordinates.

The so-called safety factor $q$ is another important quantity of MHD equilibrium. It is a measure of how tightly the magnetic field lines wrap around the torus and is defined as

$$
q=\frac{\text { number of toroidal turns }}{\text { number of poloidal turns }} .
$$



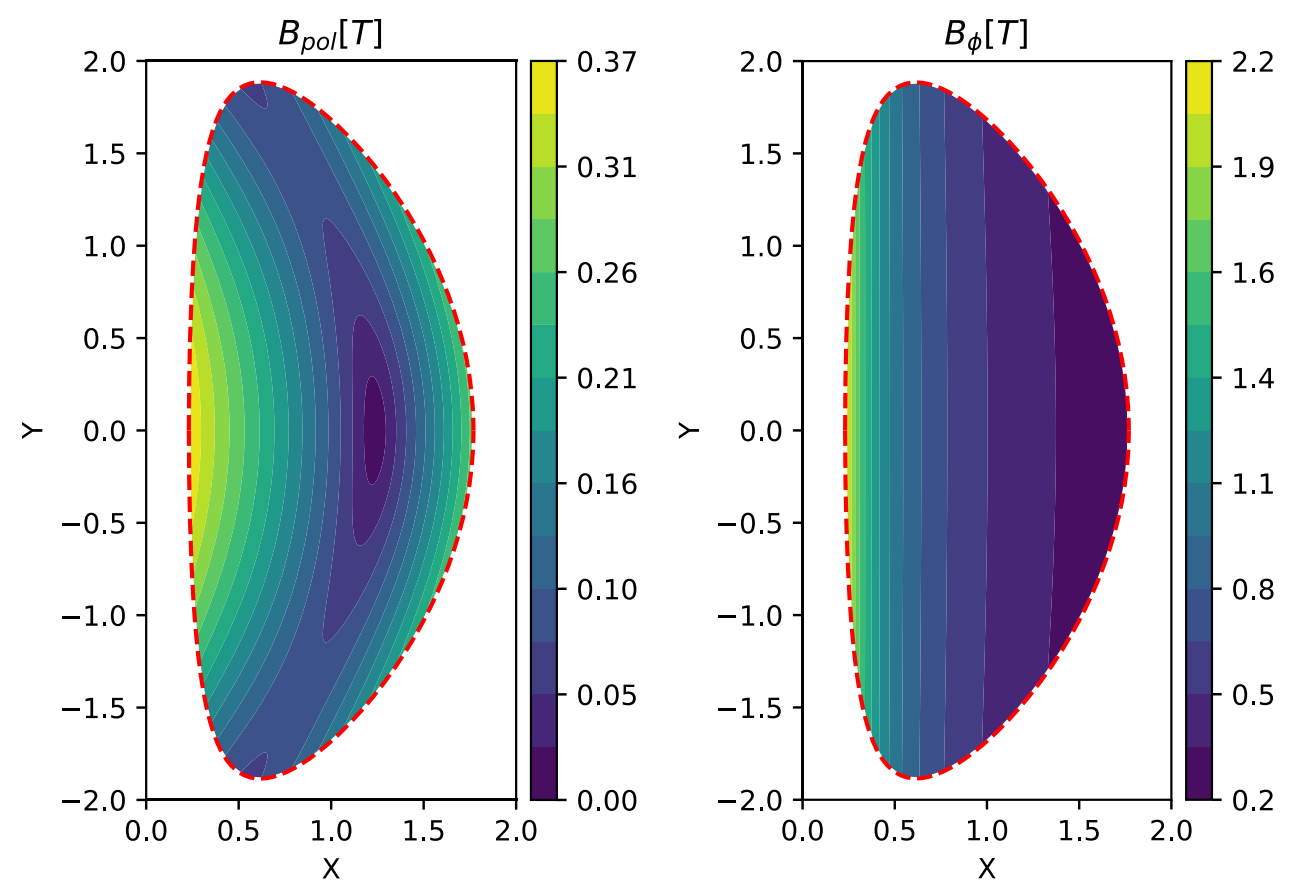

Figure 16. Magnetic field components magnitude in normalized coordinates.

As both toroidal and poloidal components of the magnetic field are known at any location, one can follow a magnetic field line and determine the safety factor using the following algorithm:

1. Starting from an arbitrary point $(X, Y)$ in the poloidal plane one finds the poloidal and toroidal component vectors of the magnetic field at that location.

2. A small step $d s_{p}$ in the poloidal plane in direction of $B_{p o l}$ is then made (the size of the step should be adjusted depending on the particular surface size, to be small relative to the contour circumference).

3. To follow the magnetic field line the displacement in the $\phi$ (toroidal) direction is then calculated to be

$$
d s_{t}=\frac{B_{\phi}}{B_{p o l}} d s_{p}
$$


leading to a change in toroidal angle

$$
d \phi=\frac{d s_{t}}{X}
$$

(since $\mathrm{X}$ is a radius of the current location).

4. These steps are repeated until the starting point is reached again.

5. $q$ can now be calculated as follows:

$$
q=\frac{\sum d \phi}{2 \pi}
$$

The safety factor $q$ as a function of starting point of magnetic filed line tracking $(r, 0)$ is shown in Figure 17.

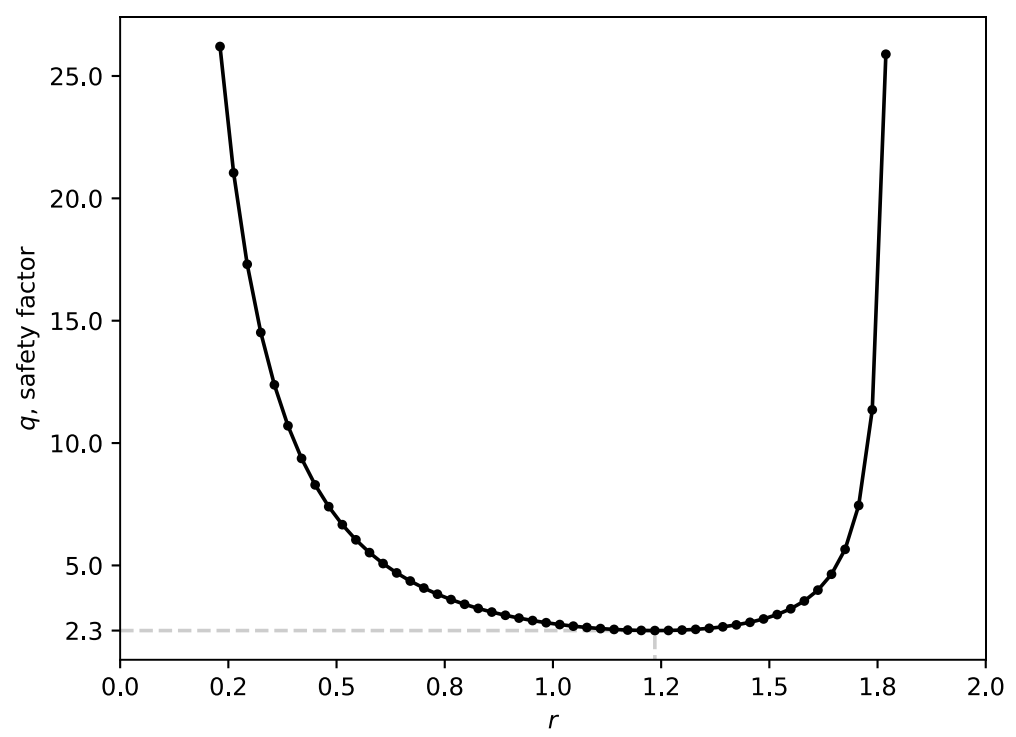

Figure 17. Safety factor profile vs. start coordinate.

Note that one finds the same values of $q$ from the left and right with respect to the magnetic axis $(r \sim 1.2, z=0)$, the point of minimum or maximum of flux function. The reason is that $q$ is constant on a flux surface and these enclose the magnetic axis. There is 
a one-to-one relationship between $q$ and $\psi$ that can be shown by plotting $q$ as a function of $\Psi$. All data points used previously lie on the same curve (Figure 18).

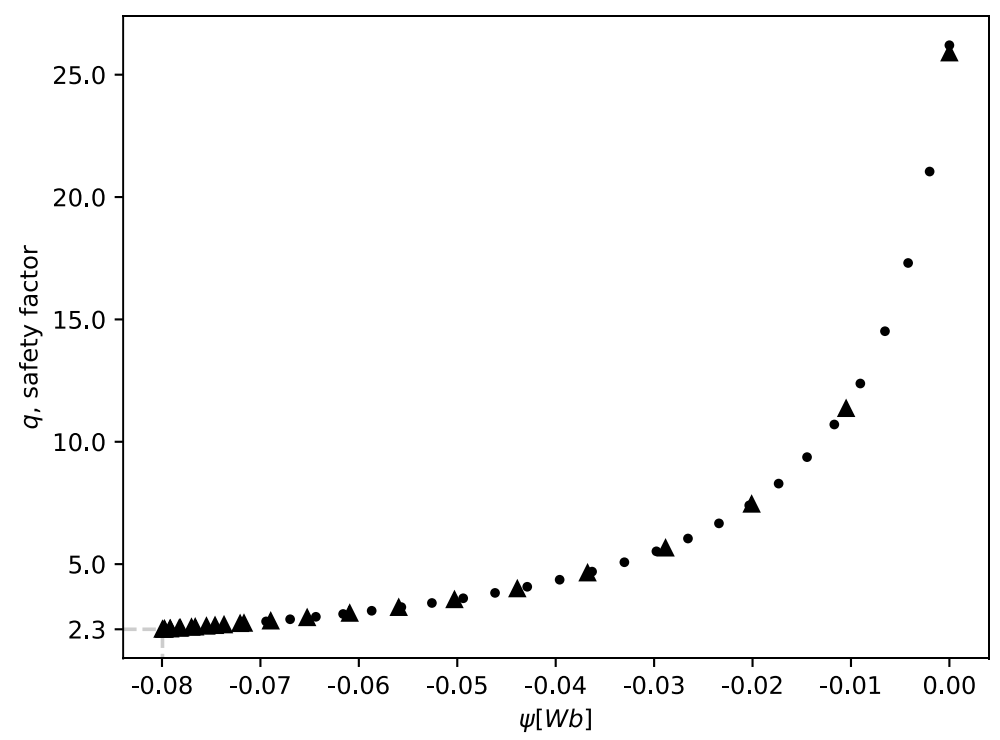

Figure 18. Safety factor profile as a function of flux value.

It is important to understand that the previously calculated current, field, and $q$ profiles do not necessarily represent the typical profiles in real machines and are a relatively simple example of a possible plasma equilibrium configuration that satisfies the Grad-Shafranov equation.

\subsection{EFIT Calculated Equilibrium}

The real plasma shots equilibrium profiles have very little in common with the one found using the Solov'ev current and pressure profiles. As can be expected, the real profiles are much more complex and thus require more sophisticated techniques for calculation.

There are various approaches to solve the Grad-Shafranov equation; the commonly used EFIT (short for equilibrium fitting) code for profile reconstruction utilizes the Green's 
function approach and Pickard Iterations described in Reconstruction of current profile parameters and plasma shapes in tokamaks by L.L. Lao [17]. EFIT uses the magnetic measurements from external magnetic probes, poloidal flux loops, as well as other diagnostics such as MSE (Motional Stark Effect), which provides the information on direction of the magnetic field lines inside the plasma, as the constraints for the GradShafranov equilibrium equation. The algorithm for the solution optimization is more complex and flexible compared to the method described in Chapter 2, but it is beyond the scope of this dissertation to go into its details. The output of EFIT reconstructed equilibrium for the future MAST-U plasma scenario is presented in the following figures.

One can notice that the flux function profile (Figure 19) looks similar to the one found in the Solov'ev method because its shape is defined by the desired geometry of the plasma cross section, which imposes constraints on the equilibrium solution.

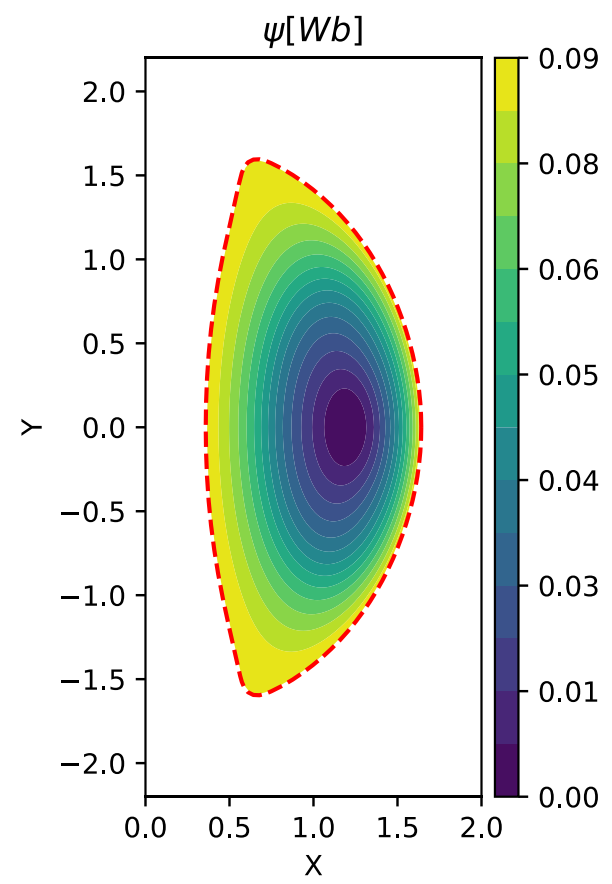

Figure 19. MAST-U EFIT calculated flux function in normalized coordinates. 
But the profiles of the magnetic field components magnitude and the current densities profiles (Figure 20, Figure 21) are very different. The difference comes from the more sophisticated constraints imposed on the magnetic field values based on the magnetic measurement data for the specific plasma shot as well as other inputs.
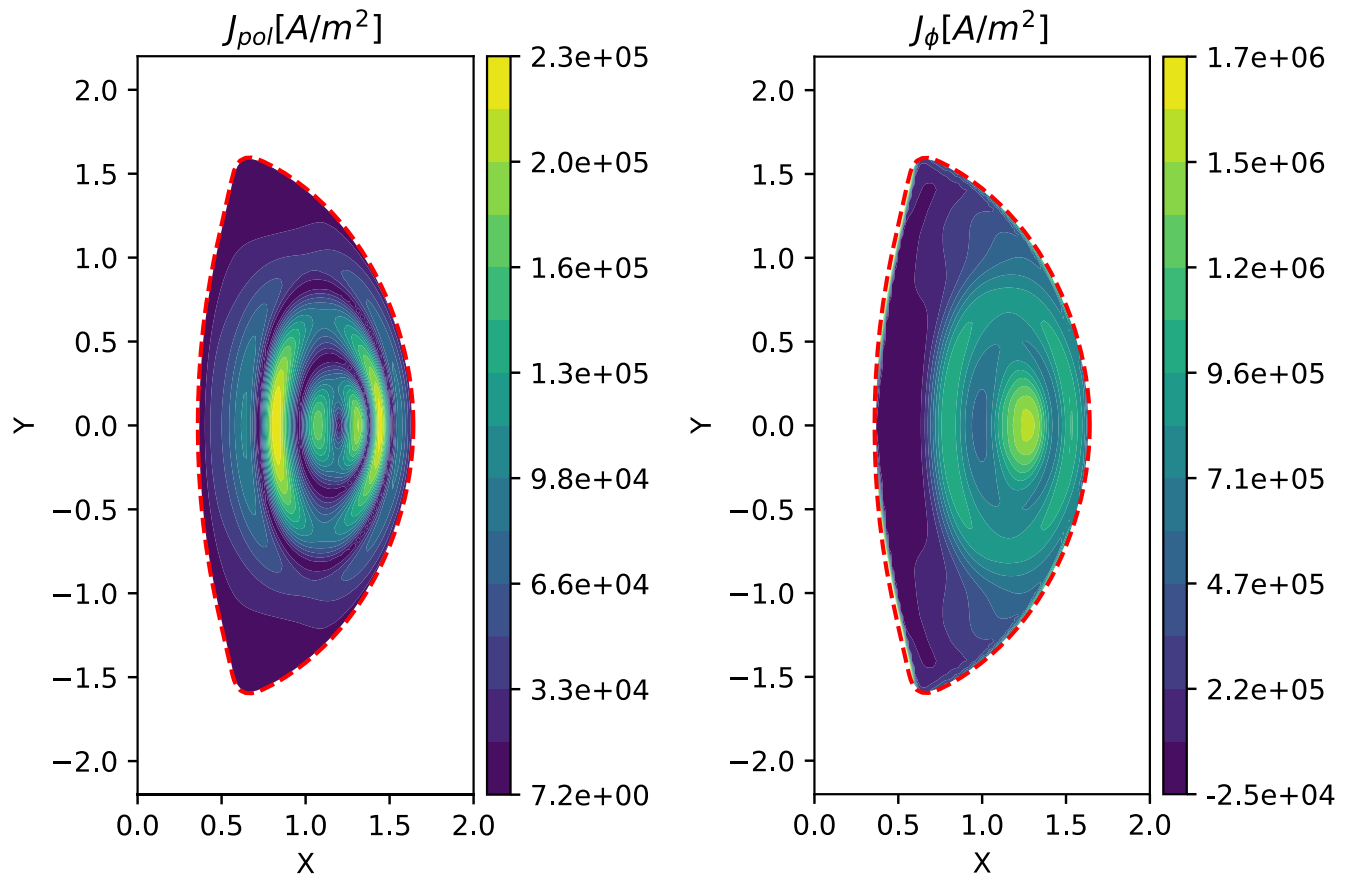

Figure 20. Poloidal and toroidal current densities (MAST-U EFIT results) in normalized coordinates.

The safety factor $q$ from EFIT calculated equilibrium shown in Figure 22 has the same type of dependence as calculated earlier with the magnetic field from Solov'ev plasma equilibrium solution, but the difference of its minimum value is quite important because of the plasma stability aspects related to the $q$ value. The higher values of $q$ typically lead to a better stability of the plasma equilibrium than with a low $q$. When the value of $q$ factor is lower than one the kink plasma instabilities can occur. 

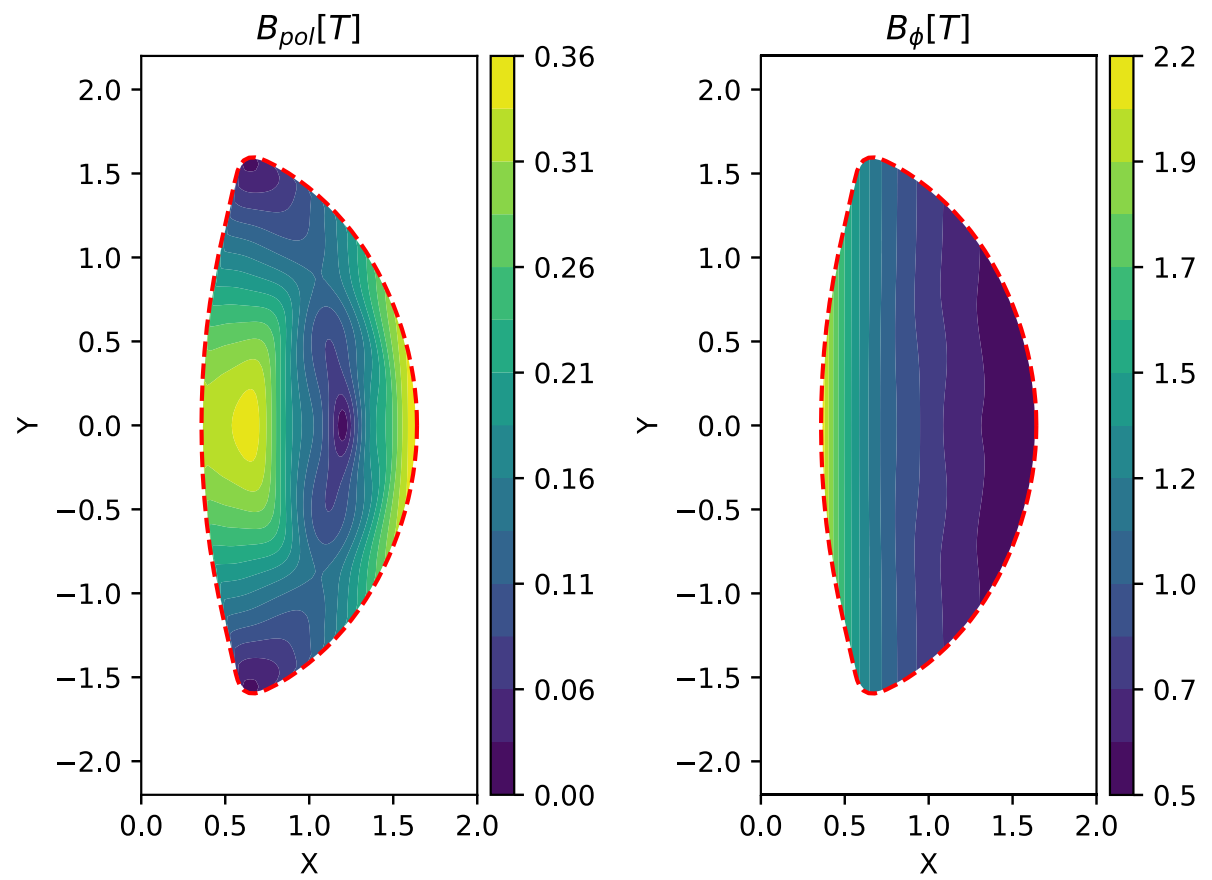

Figure 21. MAST-U magnetic field (EFIT reconstructed) in normalized coordinates.

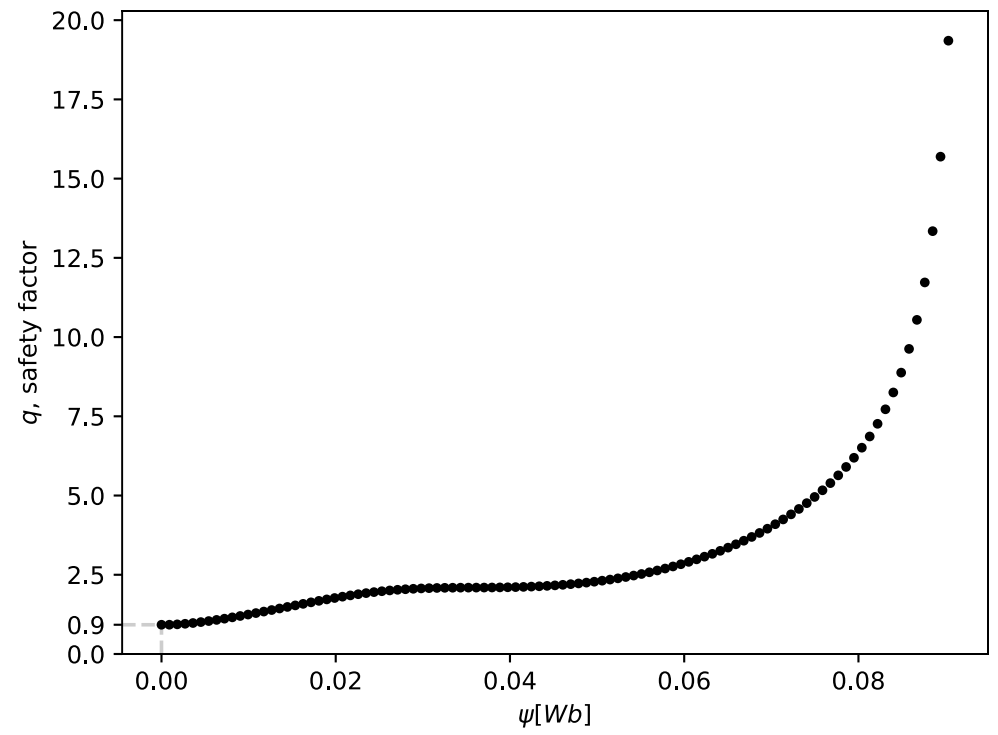

Figure 22. Safety factor $q$ as a function of flux surface defined by flux value $\psi$. 


\section{CHAPTER 3. PLASMA DIAGNOSTIC}

Fusion plasmas, at high temperature and density, exhibit very complex dynamics and various instabilities occur that can deteriorate plasma energy confinement properties even before the fusion process begins. To prevent and control these instabilities it is necessary to understand their nature and the plasma behavior in general. Plasma stability studies require a large data set on plasma parameters that can be obtained from a variety of diagnostic measurements.

Within the vast number of plasma diagnostic methods, which can be categorized according to the property or the process being measured, detection of the fusion products plays an important role in studying and understanding plasma confinement and the future plasma burning process. Two categories of fusion products can be detected from deuterium plasmas - neutrons and charged particles such as protons, tritons and alpha particles. The conducted research focuses on proton and triton detection to determine the time and position dependent fusion-reaction rate profile.

The neutral beam heated deuterium plasmas in MAST-U have a temperature of about $1.0 \mathrm{keV}$. At this temperature the D-D fusion reaction cross-section is very small resulting in a small thermal fusion rate. Most of the expected fusion reactions are due to the interaction of the plasma ions with the neutral beam particles having an energy of typically $75 \mathrm{keV}$. The D-D cross-section at this energy is orders of magnitude higher than the crosssection at the thermal energy [3]. The possible reactions for $D-D$ fusion as was mentioned earlier are $\mathrm{D}(\mathrm{d}, \mathrm{p}) \mathrm{t}$ with the release of $4.03 \mathrm{MeV}$ energy, and $D(d, n)^{3} \mathrm{He}$ with $3.27 \mathrm{MeV}$ of energy released. 


\subsection{Proton Diagnostic Principle}

In the first reaction the proton carries a kinetic energy $T_{p}=3.02 \mathrm{MeVT}_{\mathrm{p}}=3.02 \mathrm{MeV}$ and the triton kinetic energy is $\mathrm{T}_{\mathrm{t}}=1.01 \mathrm{MeVT}_{t}=1.01 \mathrm{MeV}$, neglecting the kinetic energy of the colliding reactants. Their momentum magnitudes are equal though (assuming the $\mathrm{CM}$ is at rest) and their trajectories in a magnetic field would be identical if they originate at the same position and fly in the same direction. In the second reaction, the neutron kinetic energy is $T_{n}=2.45 \mathrm{MeV}$ and the recoiling ${ }^{3} \mathrm{He}$ energy is $T_{{ }^{3} \mathrm{He}}=0.82 \mathrm{MeV}$. The protons and tritons produced are not confined and leave the plasma quickly where they can be detected.

These escaping protons and tritons can be detected using ORTEC ULTRA BU-013050-300 detectors. Each detector has an $18 \mathrm{~mm}$ outer diameter and an $8 \mathrm{~mm}$ diameter active area. A maximum depletion depth of $300 \mu \mathrm{m}$ requires a bias voltage of about $40 \mathrm{~V}$. Electron-hole pair production requires an energy of only around $3.5 \mathrm{eV} / \mathrm{pair}$. Consequently, a large number of electron-hole pairs are produced by a $3 \mathrm{MeV}$ proton, which leads to a very good intrinsic energy resolution of the detector.

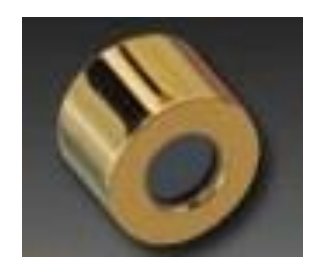

Figure 23. Ion implanted silicon surface barrier detector.

The position where the fusion reaction takes place and the direction of the produced particle define its trajectory. Assuming a known magnetic field, a time reversed calculation starting with the position and direction of the detected particle at the detector can be performed to determine the plasma region where the protons and tritons originated. 
Plasma current: $I_{p}=2 \mathrm{MA}$ Toroidal magnetic field: $B_{T}=1 \mathrm{~T}$

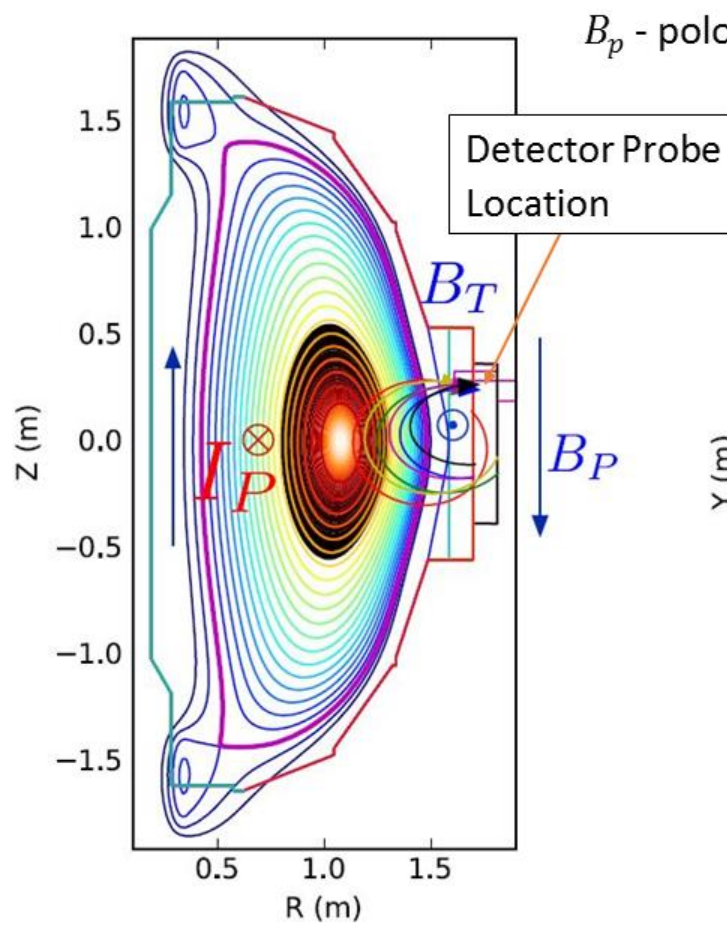

Poloidal Cross-Section View

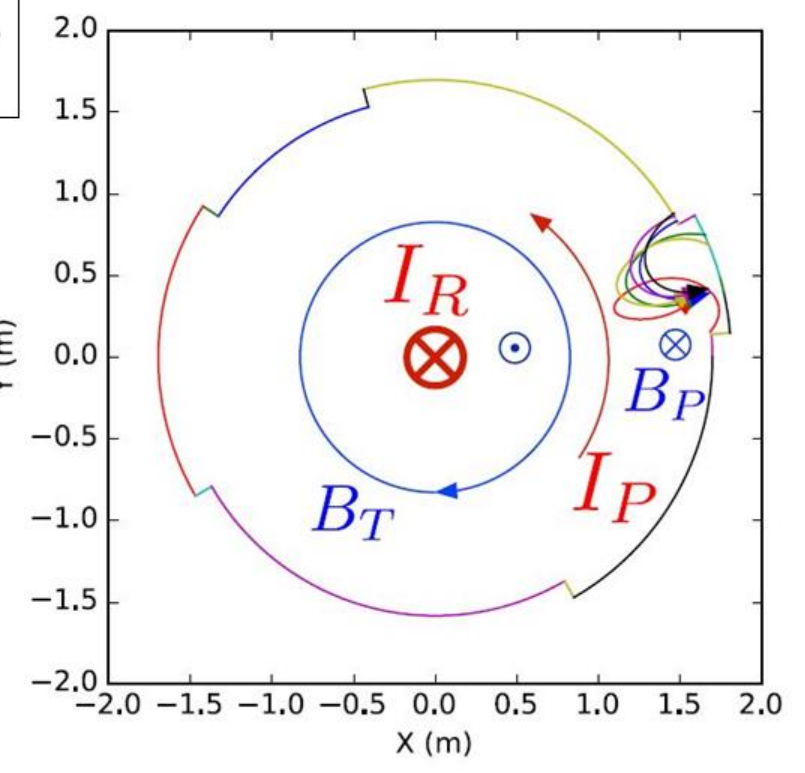

Toroidal Top View

Figure 24. Plasma probing central trajectories for NSTX-U [14].
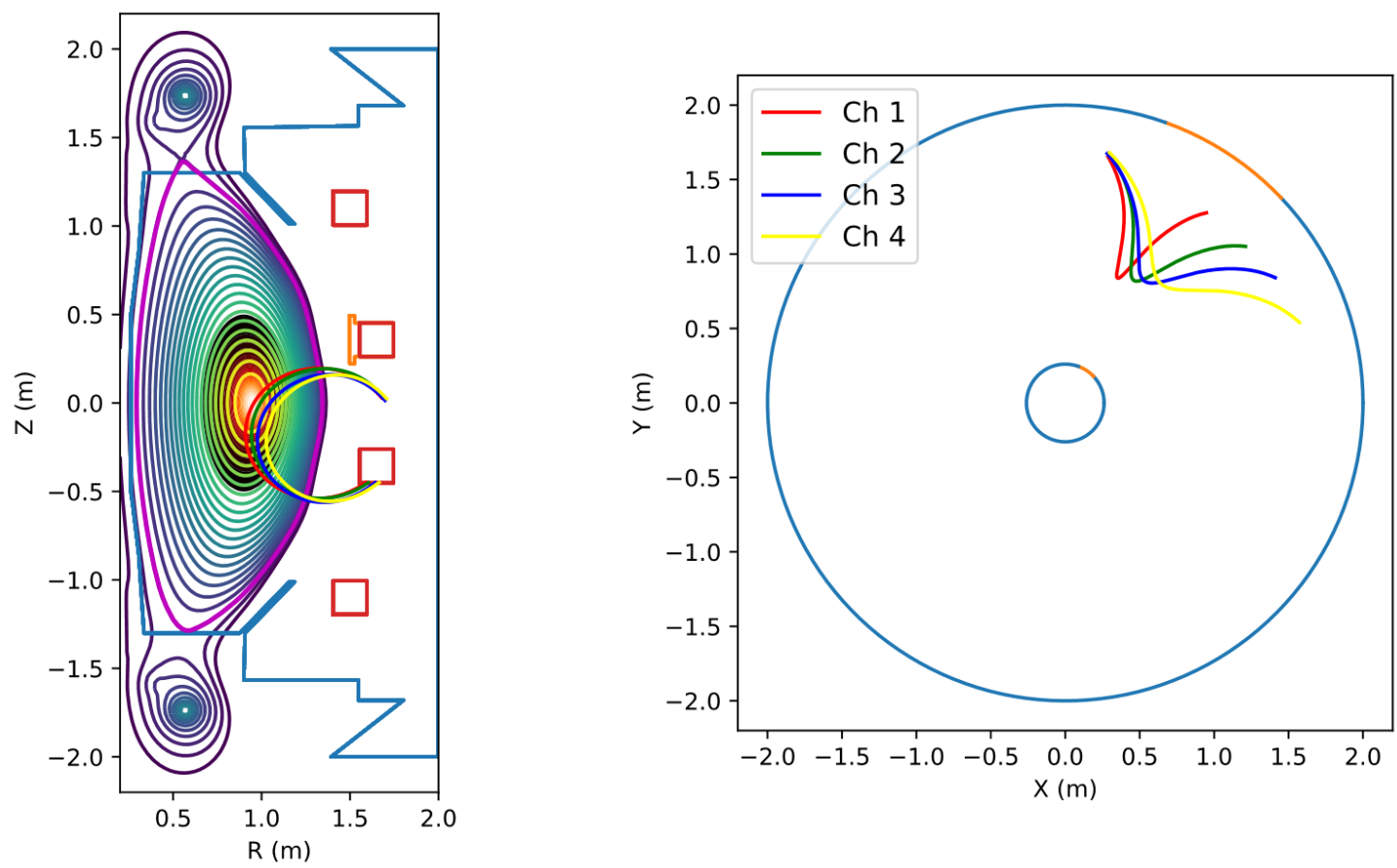

Figure 25. MAST-U 4 detector probe central trajectories. 
Central trajectories for the six-detector array are illustrated in Figure 24 together with the magnetic field and plasma current directions in NSTX-U.

The diagnostic probe head consisting of four detectors was successfully used for the MAST 2013 experimental campaign and is planned to be reused in a slightly modified version in upcoming experiments. Central probing trajectories for the planned MAST-U plasma scenario are shown in Figure 25. A detailed description of the 4 detectors probe and experiments carried out previously can be found in [19].

\subsection{Probe Mechanical Design}

A new six detector probe head has been designed for the NSTX-U charged fusion-product diagnostic and can be adapted for use at the MAST-U experiment. It consists of six detectors placed in individual metal housings with inner and outer collimators. The inner collimator is designed as a washer shaped metal insert with a $2 \mathrm{~mm}$ hole in the center and the outer collimator is a cap shaped part with a $2 \mathrm{~mm}$ opening as well. The spacing between the collimators is $35 \mathrm{~mm}$, which together with the opening size define the angular acceptance of the detector (Figure 26). The big advantage of having removable collimators is flexibility in limiting the maximum rates seen by the detectors.

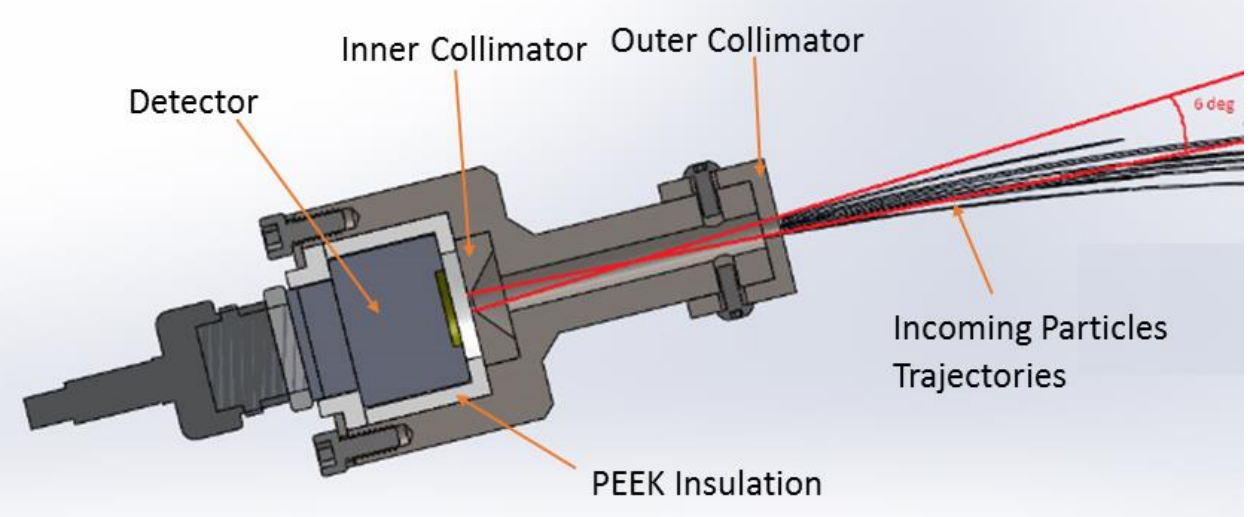

Figure 26. Detector module cut view. 
The 6-detector probe head has the detector housings mounted on a mid-plane as shown in Figure 27. The orientation of the detectors had been selected in such a way that trajectories bundles cover more or less evenly spaced regions of plasma where the neutral beams interact with the plasma.

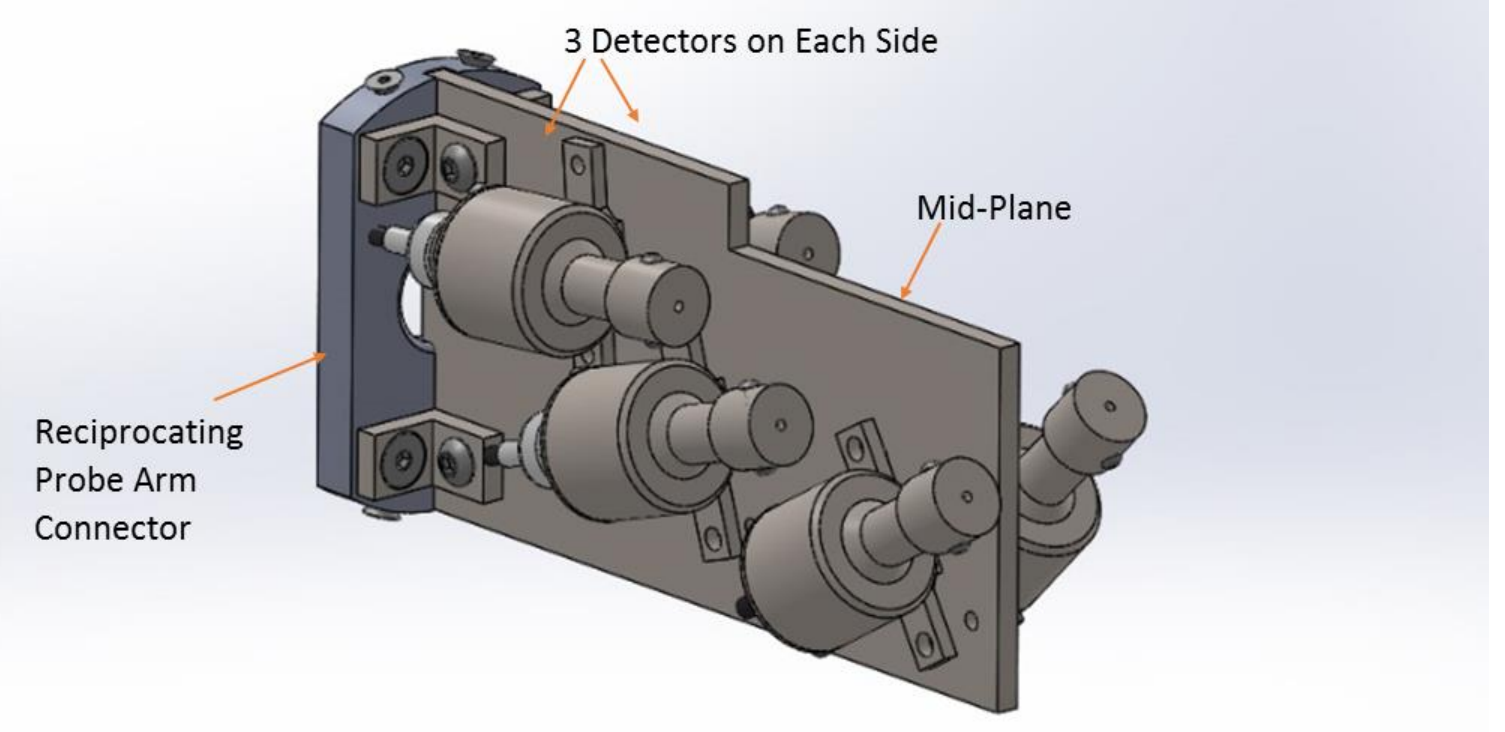

Figure 27. Detector probe design.

The detector array is covered with a ceramic coated metal shield (Figure 28) to reduce the heat load and to protect the detectors from damaging radiation in case that plasma confinement is suddenly lost. The detector head was built for NSTX-U (Figure 29), but because of unexpected operational delays it was adapted for use at MAST-U at the Culham Center for Fusion Energy in the UK.

The number of detectors on the probe head is mostly limited by the physically available space allowed by the small diagnostic flange aperture at NSTX-U, along with the detector orientation requirements to avoid mutual blocking. For the larger opening diagnostic port available at MAST-U an extended midplane design was studied with 8 detectors (Figure 
30). The core plasma coverage of such system is shown in Figure 31 . The new data acquisition electronics has a capability of acquiring data from up to 16 channels, so the diagnostics can be further extended to obtain more detailed spatial information of the fusion emissivity profile.

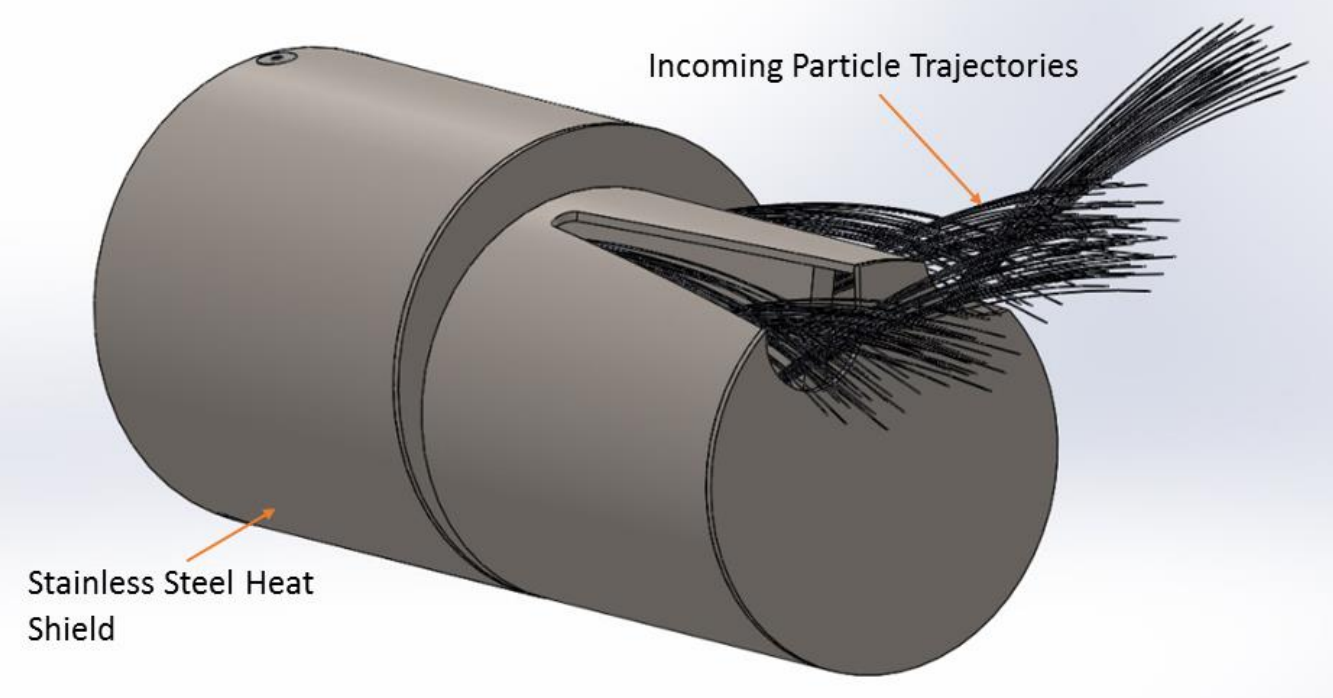

Figure 28. Detector probe with heat shield.

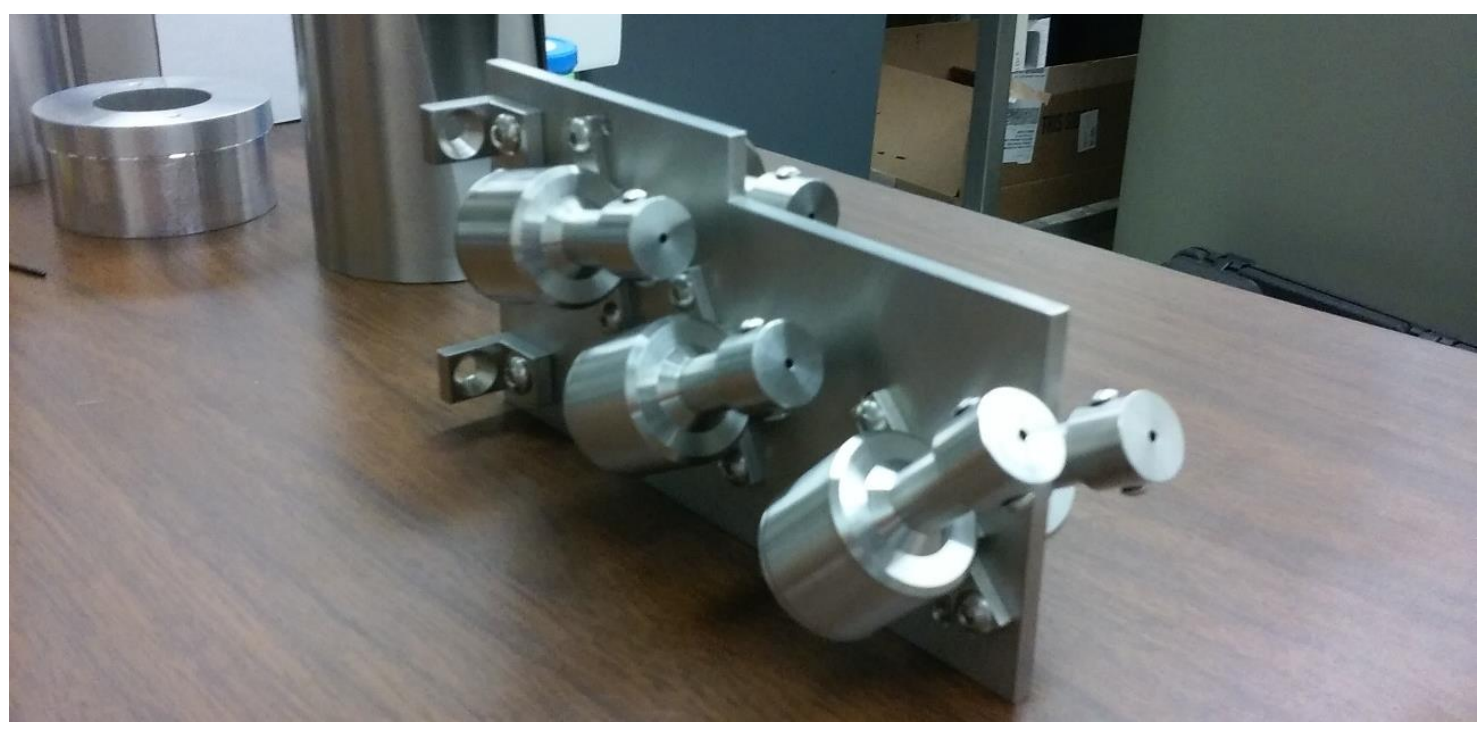

Figure 29. Machined 6-detector probe head (stainless steel SS316). 


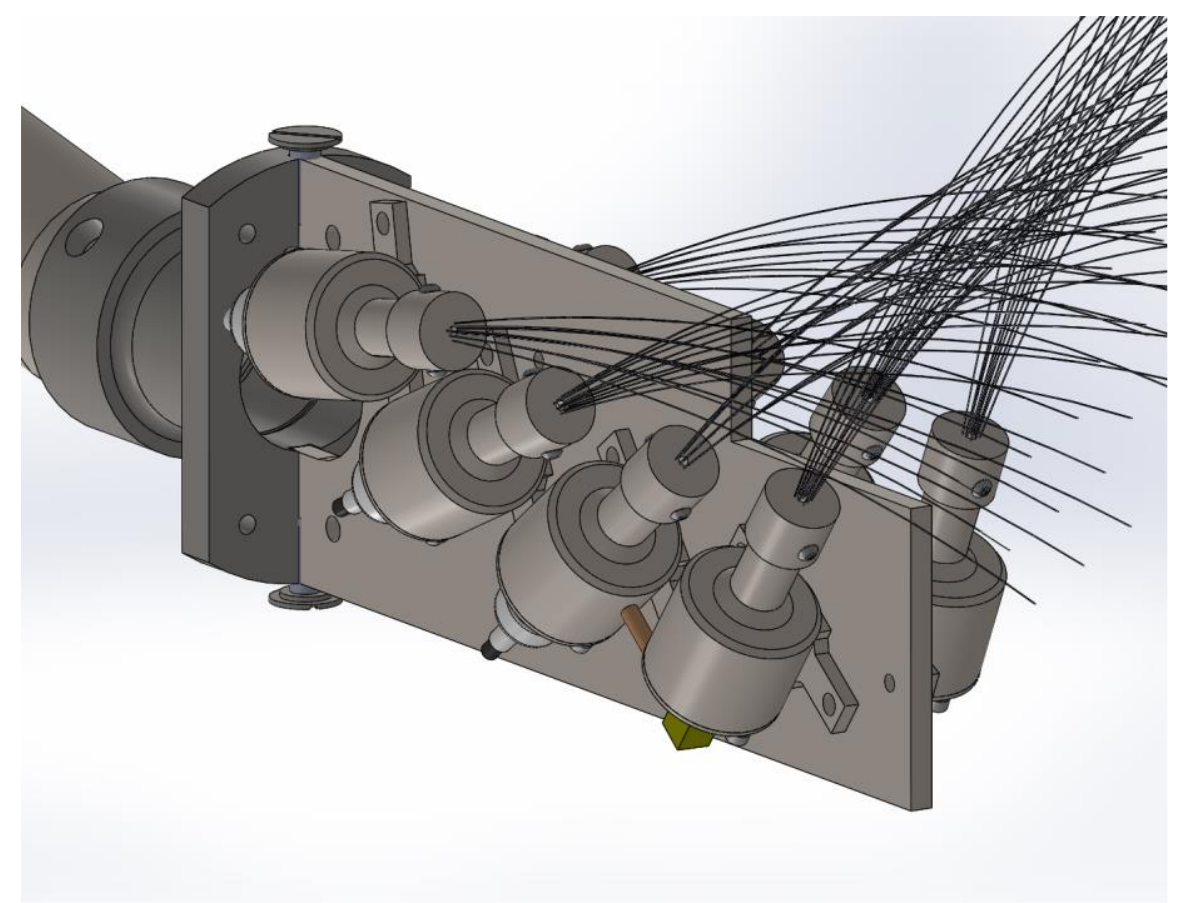

Figure 30. 8 detector probe (heatshield is not shown), with probing trajectories.

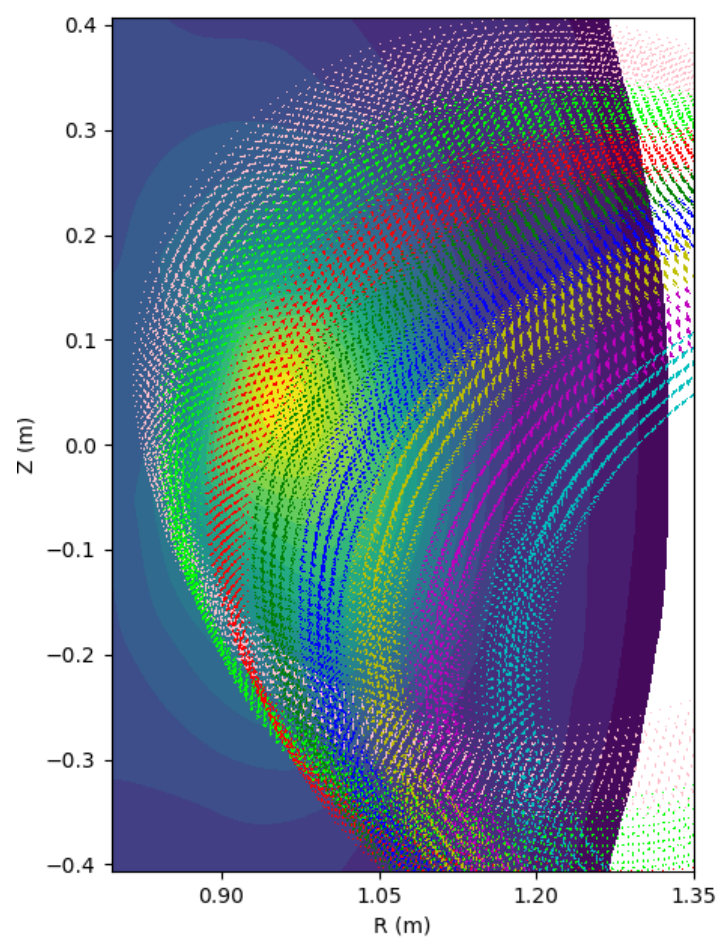

Figure 31. MAST-U 8 channel probing bundles with fusion emissivity profile shown as a background. 
The probe head is mounted on the support arm, which has a retraction and rotation motion range for plasma probing flexibility, the fixed support arm was also considered for cost savings or in case of reciprocating arm unavailability. The retractable support arm designed for NSTX-U has the advantage of being equipped with a vacuum gate, thus the probe can be retracted and serviced if needed (changing the collimators, etc.) without breaking the main vessel vacuum (Figure 35).

As can be seen from the mechanical design of the detector housing (Figure 26), each detector accepts incoming particles within a range of incident angles. The accepted angle range together with position and orientation of the detector define its plasma probing region, which can be calculated by the time reverse calculation of the trajectories starting from the detector.

The plasma regions probed by each detector/collimator pair for the same plasma parameters as in Figure 24 are shown in Figure 32. The thickness of the bundles depends on the angular acceptance of the detectors, which is determined by the collimators. Replaceable collimators allow one to adjust the acceptance width and the detector's particle load. These probing regions can be remotely changed (Figure 33, Figure 34)via axial rotation and radial retraction capabilities of the reciprocating probe arm supporting the probe head inside the vacuum vessel (Figure 35, Figure 36). 


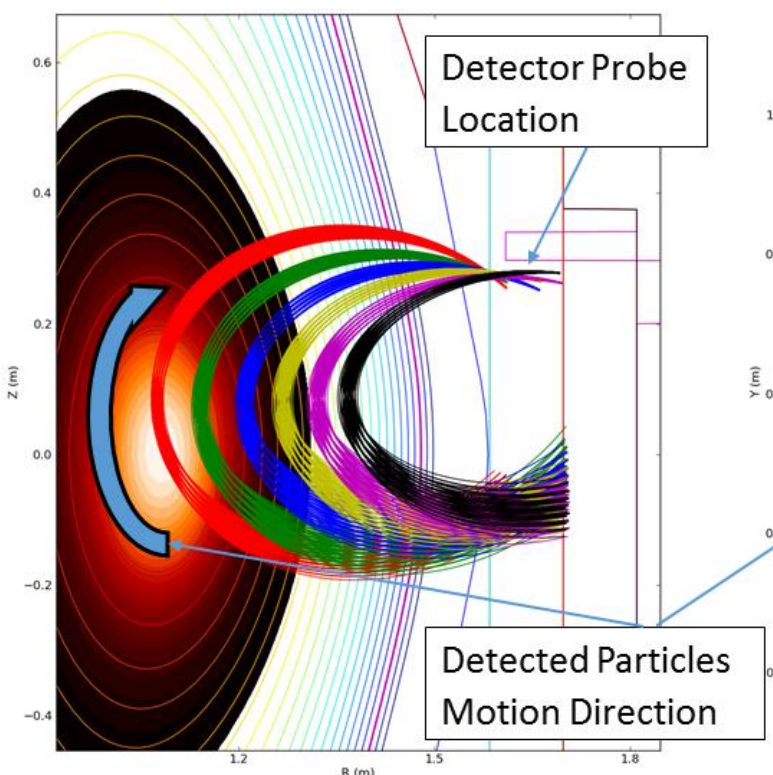

Poloidal Cross-Section View

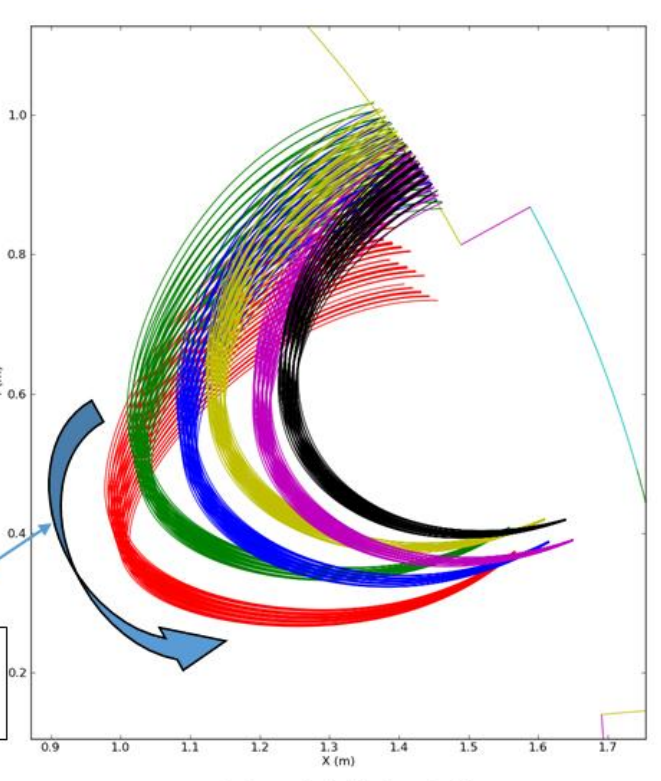

Toroidal Top View

Figure 32. Plasma probing trajectories bundles.
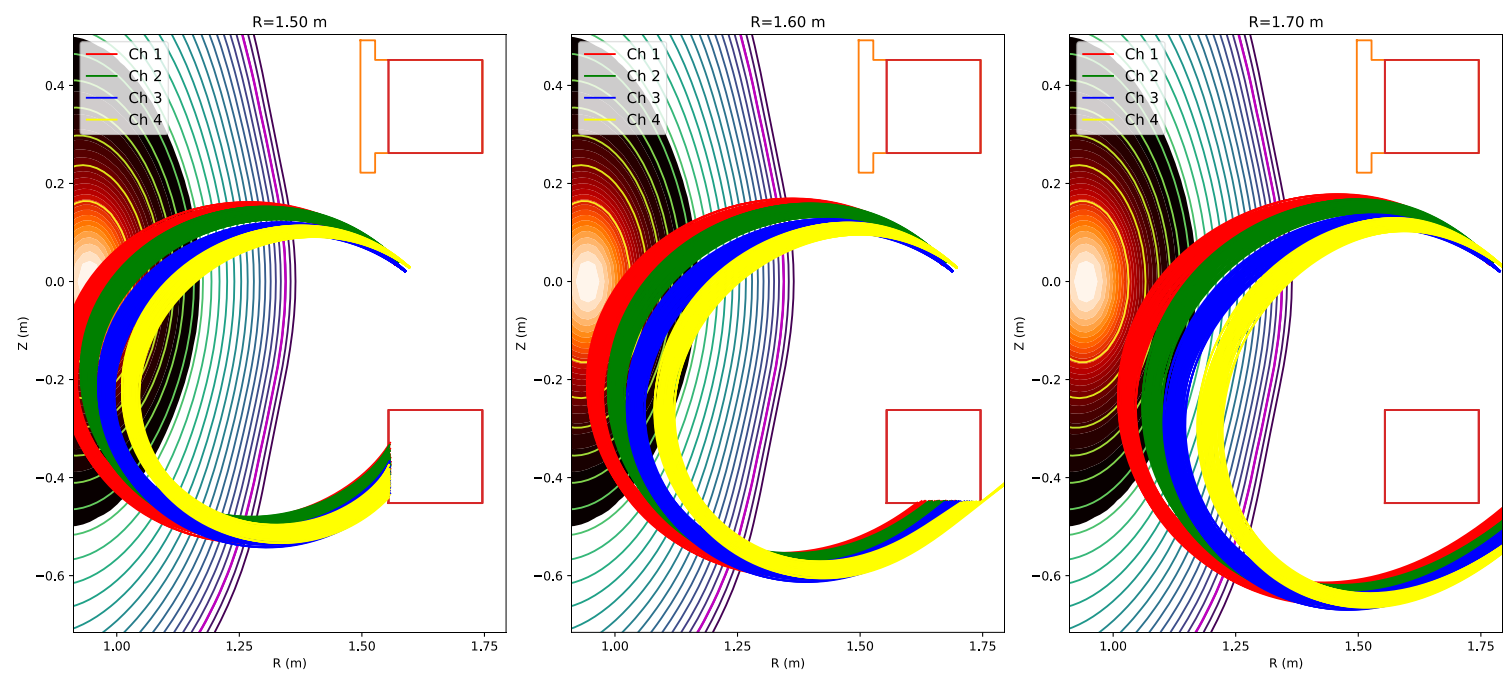

Figure 33. Probing trajectory bundles for varying radial head position (varying distance from the tokamak center to the probe). 

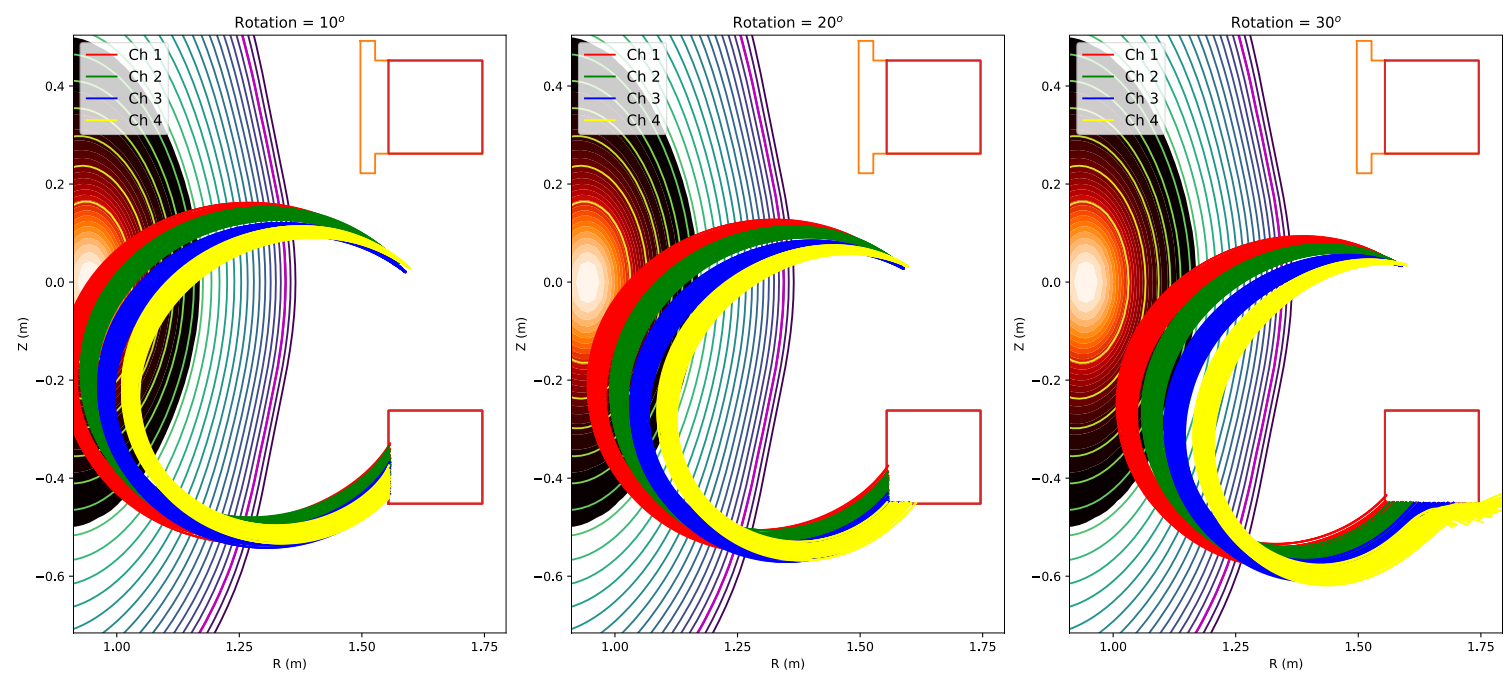

Figure 34. Probing trajectory bundles for varying axial head rotation.

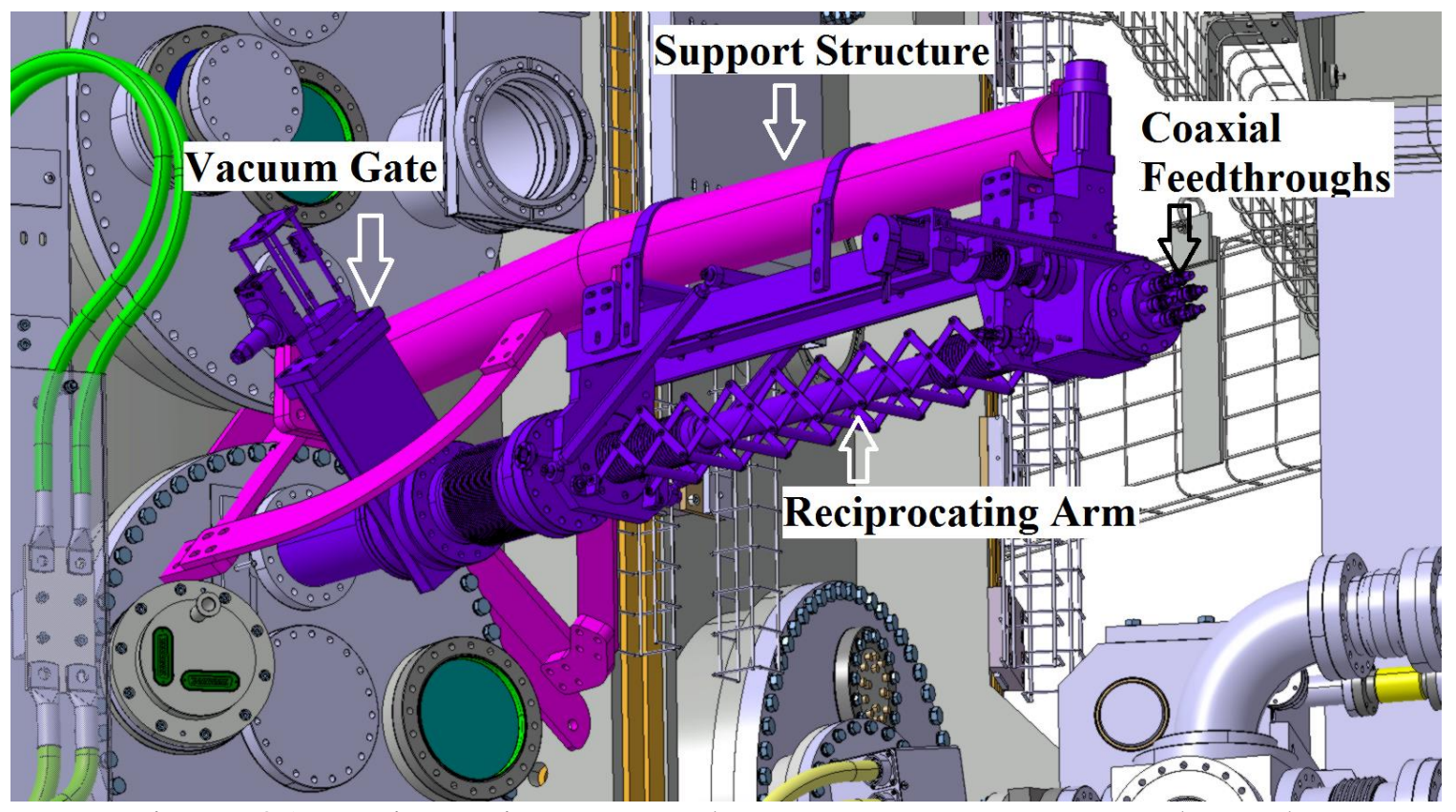

Figure 35. Reciprocating arm and support structure mounted on the diagnostic port outside of the vessel (requires adaptation for MAST-U). 


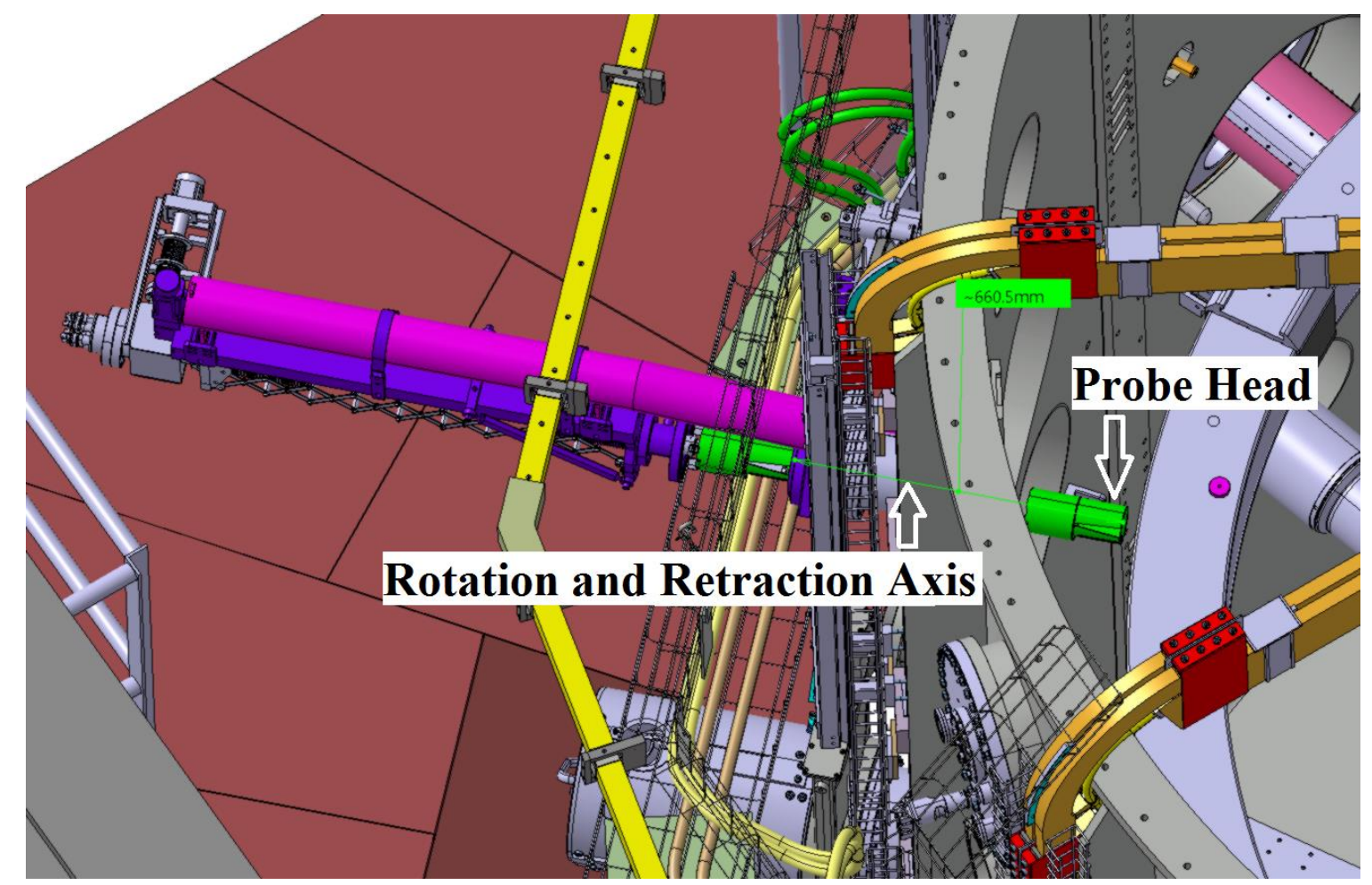

Figure 36. Reciprocating arm retraction range (probe head shown in green).

The calculation of the probing trajectory bundles given a known equilibrium magnetic field configuration is an essential part of the charged fusion product diagnostic. First of all it gives a spatial information of the measured particle rates, and also it is necessary for predicted rate calculations given a modeled emissivity. This then allows comparison of the predicted and measured rates and validation of the emissivity profile.

The 4-detector probe design used in the MAST 2013 experimental campaign had no removable collimators. Thus the adaptation for the increased power injected by the neutral beams and the higher expected rates was performed, collimating inserts were designed and are shown in Figure 37 together with a cross-sectional view of the existing 4-detectors probe head [19]. These inserts have an inner opening size determined by rate calculations 
to limit the particle count rates below $200 \mathrm{kHz}$ per detector, which is a safe working limit for the detectors to avoid electrical overload and signal pileup.

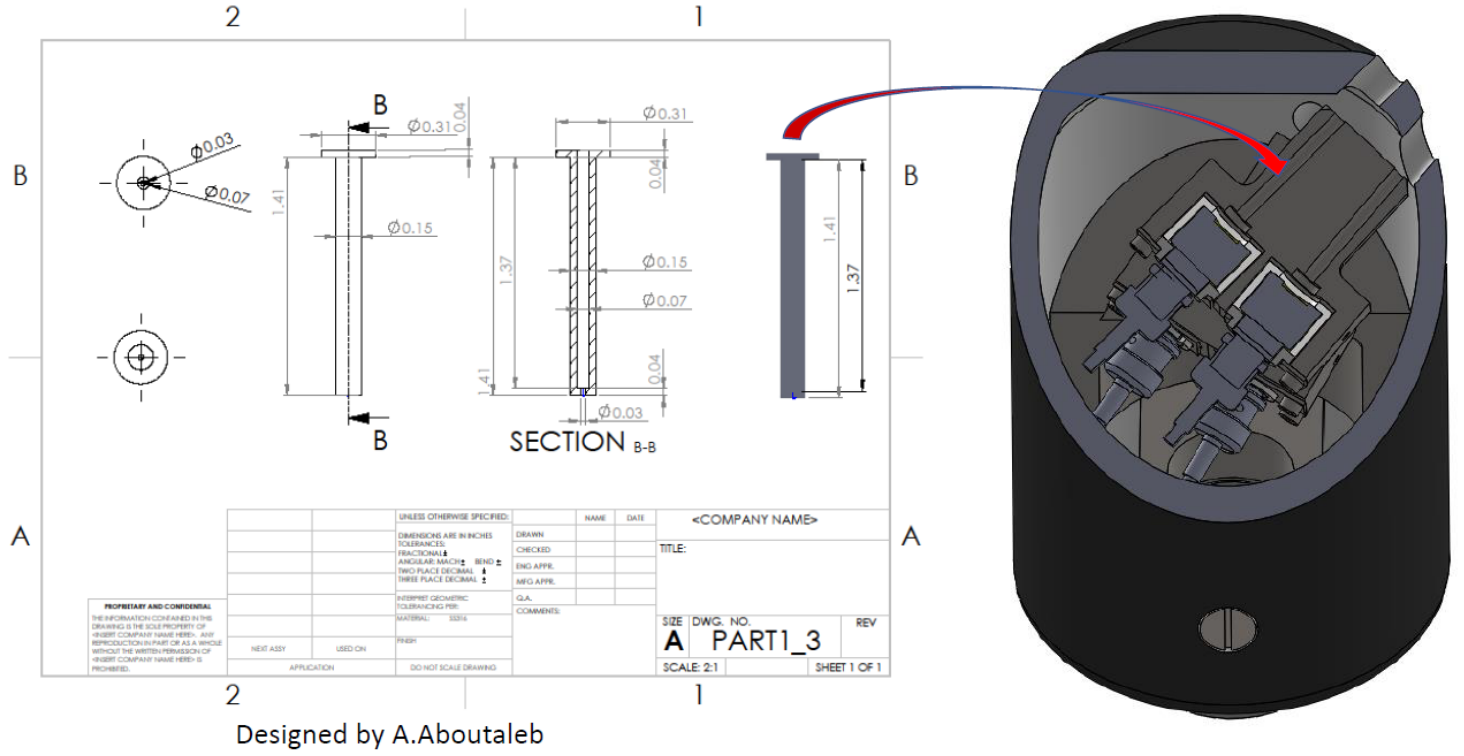

Figure 37. Collimator inserts for 4-detectors probe head.

\subsection{Effect of Trajectory Curvature Inside the Detector Housing}

Charged fusion product trajectories necessary to determine the plasma regions being probed by each detector are calculated by the ORBIT code. It was developed at PPPL [26] and upgraded at FIU. The trajectories of fusion products are calculated by numerically solving the equation of motion of a charged particle in the equilibrium field obtained from the solution of Grad-Shafranov equation. Given the particle mass, charge, and initial velocity vector along with the magnetic field configuration, the ORBIT code calculates the forward or time reversed track of the particle (the time reversed calculation is done by reversing the particle velocity vector and magnetic field vector). For our detectorcollimator system the initial velocity vector can be any vector within the solid angle defined by the acceptance. To calculate the probing trajectory bundles, the splitting technique is 
applied, where the detector and collimator surface are represented either as a rectangular grid with equal-square subsections, or a circular grid with sectors of equal areas (Figure $38)$.
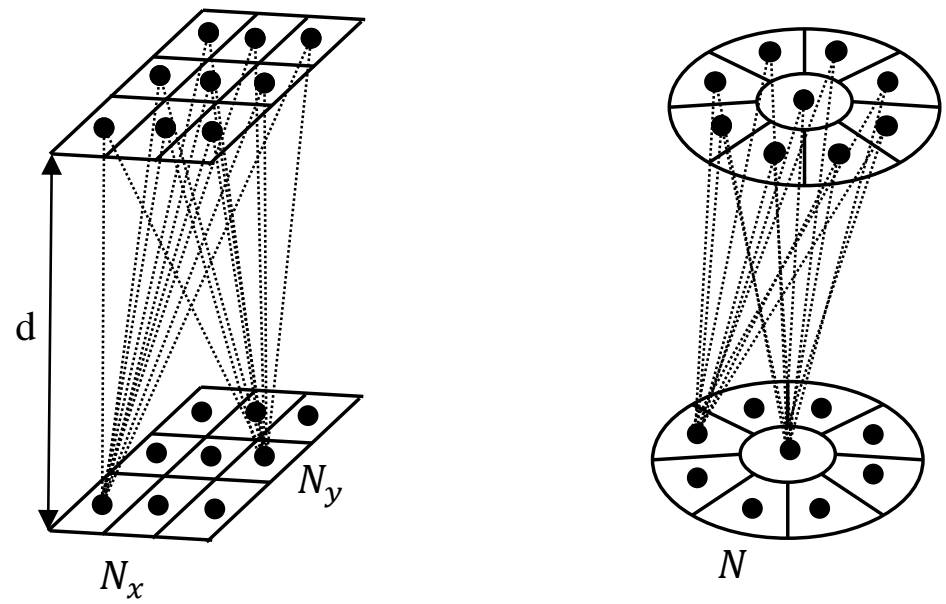

Figure 38. Collimator/detector schematic splitting for bundles calculations.

The center point of each element of the detector area is then connected to collimatorsector centers in all possible combinations giving the set of all possible trajectories for the given detector collimator pair. In the case of a disk-shape splitting on $k$ elements, the inner central round segment should have a radius of $r_{c}=r / \sqrt{k}$ in order to have the same area as the rest of the segments. The possible trajectory directions are first calculated in the coordinate system of the detector, and then transformed into the tokamak coordinate system to form the input file of the ORBIT code. The acceptance of each segment pair combination can be calculated as

$$
A_{i j}=\frac{S_{i} S_{j} \sin ^{2} \theta_{i j}}{d_{i j}^{2}},
$$


where $S$ is a segment area, $\theta_{i j}$ is an angle between detector/collimator direction $\vec{d}$ and the vector connecting two selected segments $\vec{d}_{i j}$.

A trajectory is started at the collimator exit with the initial velocity direction defined by the vector $\vec{d}_{i j}$, Figure 39 a). Since our detector housings are made of non-magnetic material, the trajectory from the detector to the collimator exit is not shielded from the magnetic field and hence is curved. For accurate probing bundle calculations the effect of the mentioned curvature needs to be included in the trajectories' initial values at the exit of the collimator. Neglecting the magnetic field in the collimator the initial value of the velocity direction (unit vector) is given by the central detector-collimator sightline. However, it would be inaccurate to start the trajectory from the detector since as that trajectory would be possibly trapped inside the detector housing (Figure $39 \mathrm{~b}$ ). On the average, the magnetic field inside the detector-collimator leads to an effective overall average deflection of the trajectories with respect to the central line and perpendicular to the magnetic field. This angle can be calculated from the Lorentz force integral over distance $d$ with the small angle approximation:

$$
\alpha=\frac{q[\vec{v} \times \vec{B}]}{m v^{2}} \frac{d}{2},
$$

where $d$ is the detector to collimator distance. Thus, the initial velocity vector of the trajectory which starts from the outer collimator should be tilted by the angle $\alpha$ as shown in Figure 39 c). 


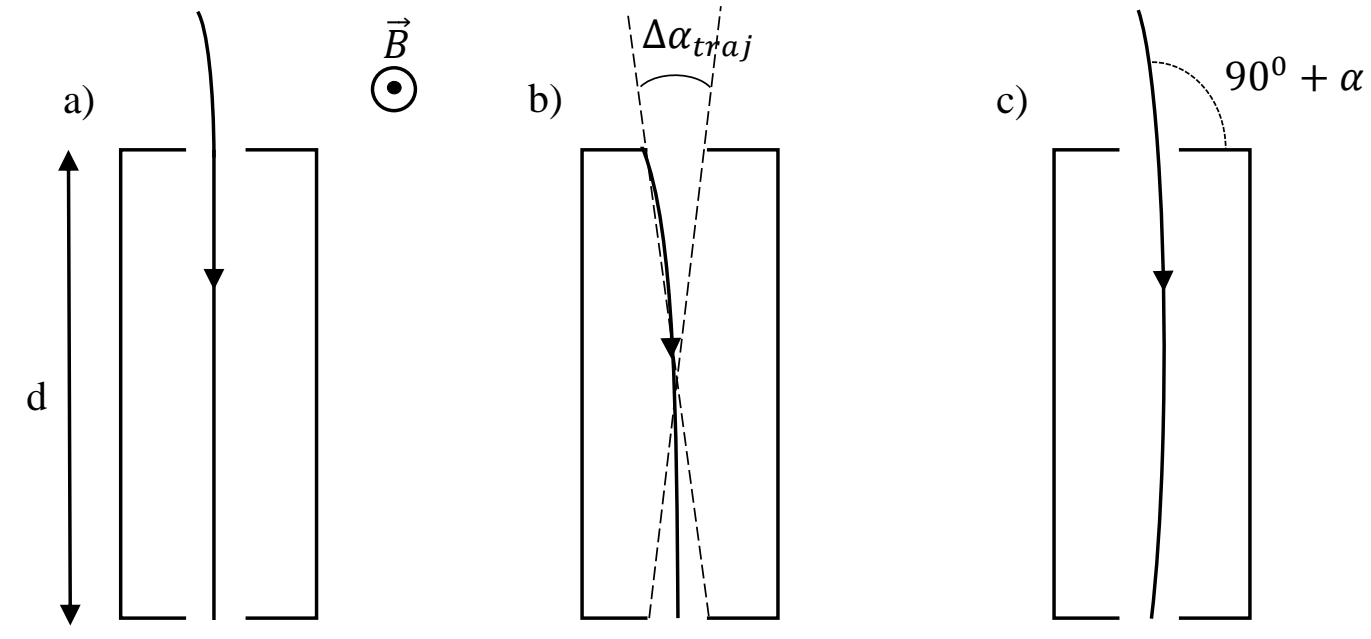

Figure 39. Trajectory curvature on detector - collimator distance.

A frequently used characterizations of the probing trajectory is its crossing point with the tokamak mid-plane since the emissivity distribution peak is typically located on the midplane (Figure 40). One can estimate how this crossing point shifts when the additional angle $\alpha$ is introduced to the trajectory starting direction (Figure 41). As an approximation the poloidal projection of the trajectory can be represented by a circle with a radius $r_{p} \cong$ $50 \mathrm{~cm}$, which is the radius of a $3 \mathrm{MeV}$ proton in a $0.65 \mathrm{~T}$ toroidal magnetic field with its velocity perpendicular to the field. The calculated deflection angle $\alpha$ in this case is about $2^{0}$, which is comparable to the trajectory angle spread range (Figure $39 \mathrm{~b}$ )):

$$
\Delta \alpha_{t r a j}=\frac{4 r_{c}}{d} \cong 6^{0}
$$

where $r_{c}$ is the collimator opening radius $(\sim 2 \mathrm{~mm})$, and $d$ is the collimator/detector spacing $(\sim 35 \mathrm{~mm})$. Assuming that the detector is located on a midplane, the shift in the midplane crossing point in the small angle approximation is 


$$
\Delta r=r_{p} \alpha^{2}=\frac{d^{2}}{4 r_{p}}
$$

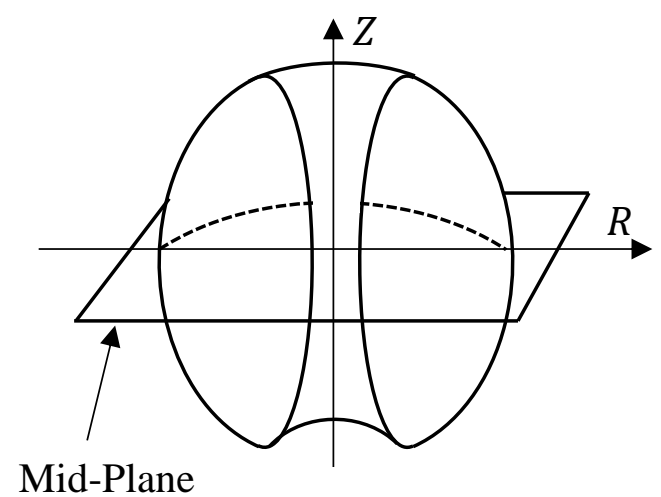

Figure 40. Spherical Tokamak Mid-Plane $(Z=0)$

For a detector - collimator spacing of $3.5 \mathrm{~cm}$ the shift is $\sim 1 \mathrm{~mm}$. It should be noted that this approximation assumes a uniform magnetic over the entire trajectory span.

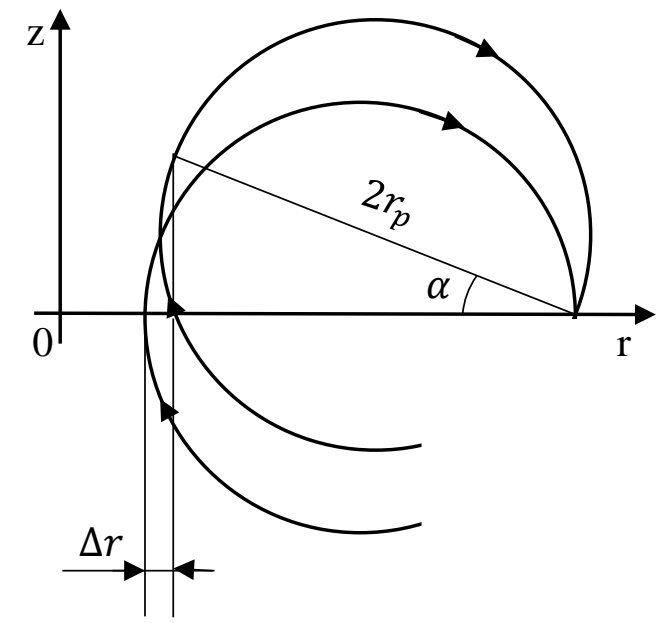

Figure 41. Midplane crossing shift due to trajectory direction correction angle $\alpha$ in a uniform magnetic field.

The tokamak magnetic field is highly nonuniform and this shift, calculated using ORBIT, appears to be much bigger and should be taken into consideration. In Figure 42 the midplane crossing for various trajectory bundles are shown in the form of histograms, 
where the horizontal axis is the midplane crossing radial coordinate, and the bin content represents the number of the trajectories crossing the mid-plane within the bin width. Each bundle consists of 81 trajectories since the $3 \times 3$ segments splitting is used for the detector and collimator representation. Solid vertical black lines show the mean value of the crossing radius for each bundle, and the dashed lines represent the bundle midplane width calculated by the crossing radii variance. It can be noted that the bundle shift caused by the angle correction of the starting trajectories is of the order of $1 \mathrm{~cm}$, which is the same order as a bundle size. If the plasma emissivity profile has a significant spatial gradient, such that its change on the $1 \mathrm{~cm}$ scale is notable, the shifts can lead to a significant difference in the calculated predicted rate and needs to be properly considered.

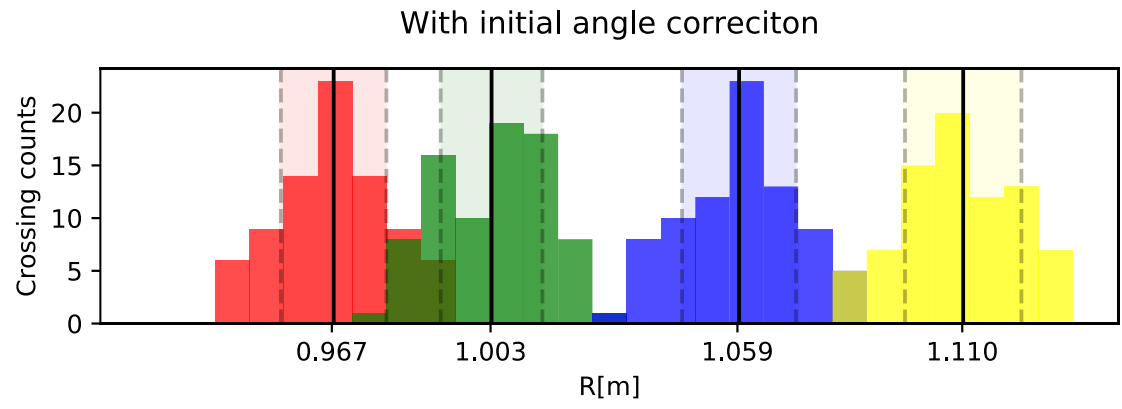

Without angle correciton

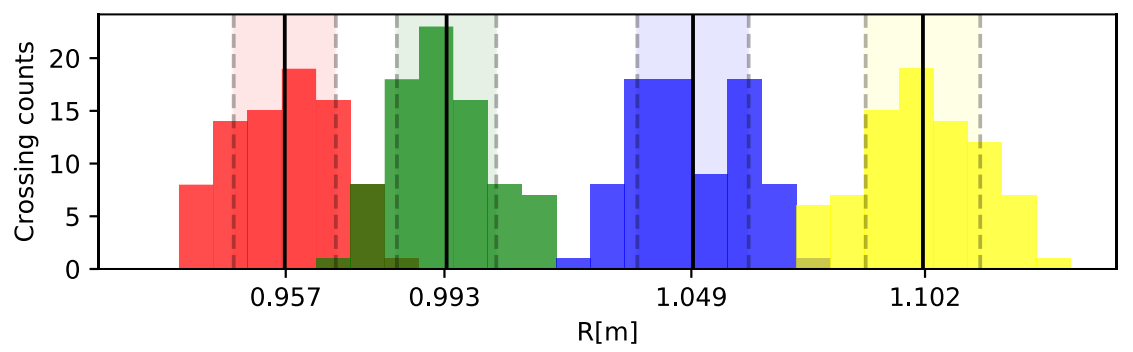

Figure 42. Trajectories bundles midplane crossing with and without correction.

The predicted rates seen by the 4 detector probe head with probing bundles calculated using the MAST-U planned plasma equilibrium EFIT data and a TRANSP simulated 
emissivity profile for the expected plasma scenarios are shown in Figure 43. The calculated rate difference caused by the angle correction in this case is not very significant since the emissivity is not changing dramatically on the calculated bundles shift scale. The rate error bars correspond to the statistical uncertainty in the count rate with an integration time of $\Delta t=1 \mathrm{~ms}$.

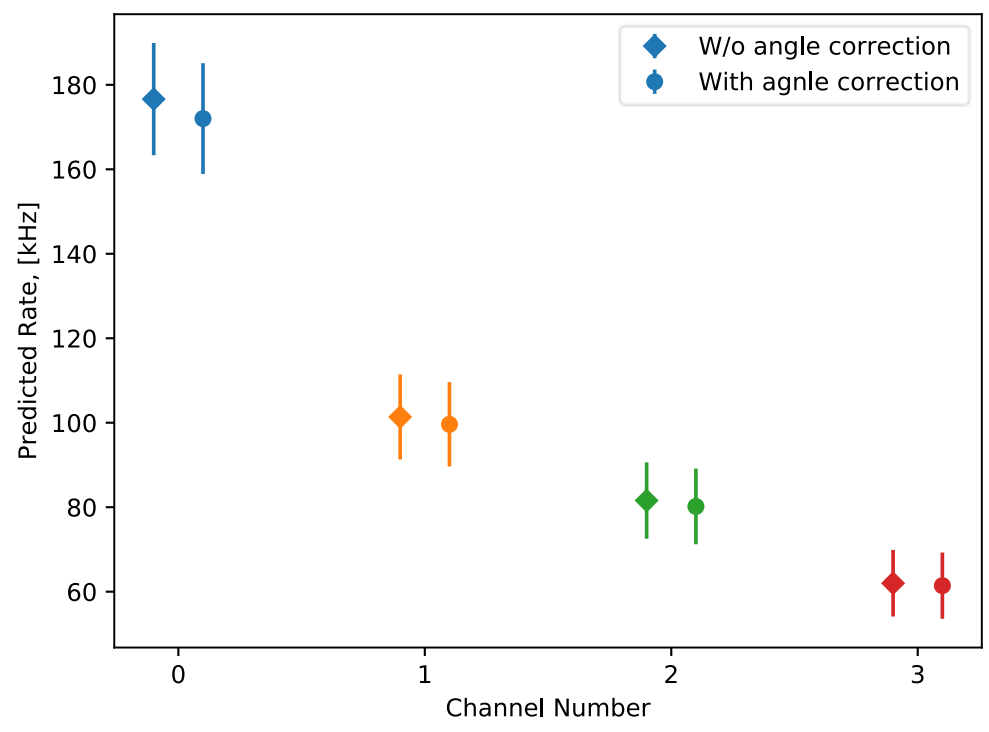

Figure 43. Predicted rate change due to starting trajectory angle correction. 


\section{CHAPTER 4. DETECTOR ACCEPTANCE AND RATE PREDICTION}

The goal of this chapter is to describe the procedure of calculating the particle flow rate seen by the detector based on a TRANSP (PPPL plasma fluid transport code) predicted emissivity profile and the physical acceptance of the detector. For the demonstrative purposes, it is convenient to consider the $2 \mathrm{D}$ motion problem at first and then move to the 3D case. This can be helpful since the phase space of the 3D motion contains 6 coordinates, which makes it hard to visualize and intuitively feel certain effects related to phase-space volume conservation during the motion. The $2 \mathrm{D}$ motion is described by the trajectories in 4D phase space in the general case, while for the motion in a purely magnetic field, where the particle speed stays constant, it can be reduced to 3 coordinates by choosing position coordinates $x$ and $y$, as well as the velocity vector's polar angle as the generalized coordinates. These coordinates do not form a canonical set since the equations of motion are not canonical Hamiltonian equations. Nevertheless, this set of coordinates is convenient to use to find certain conserved quantities.

One can consider a linear detector with the active area located on the $x$-axis, and linear collimator parallel to the $x$-axis at $y=y_{d}$ (Figure 44). For each point $x$ on the active surface the acceptance angle ranges from $\varphi_{0}(x)$ to $\varphi_{1}(x)$ are given by

$$
\varphi_{0}(x)=\tan ^{-1}\left(-\frac{x}{y_{d}}\right) ; \quad \varphi_{1}(x)=\tan ^{-1}\left(\frac{x_{d}}{2 y_{d}}-\frac{x}{y_{d}}\right)
$$

This relation defines our detector acceptance as a surface in the $x-\varphi$ plane bounded between $\varphi_{0}(x)$ and $\varphi_{1}(x)$ lines when $x$ runs from $-x_{d} / 2$ to $x_{d} / 2$. A ratio of $x_{d} / y_{d}=2$ was chosen in Figure 45 to highlight that the upper and lower borders are not straight lines (normally $x_{d} / y_{d} \ll 1$ and the phase space shape in this case is simply a parallelogram). 


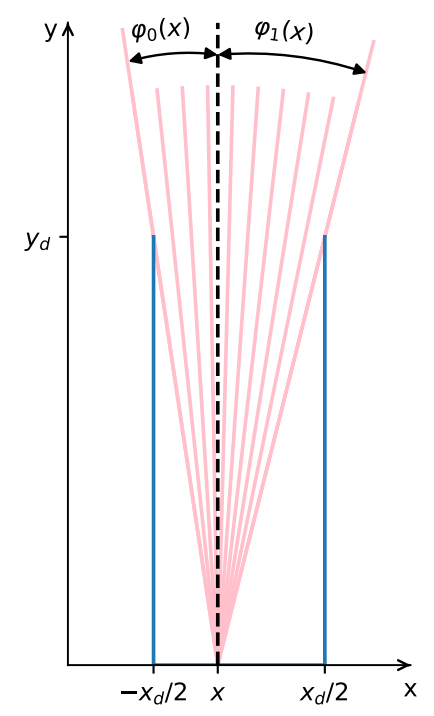

Figure 44. Detector acceptance calculation geometry in 2D.

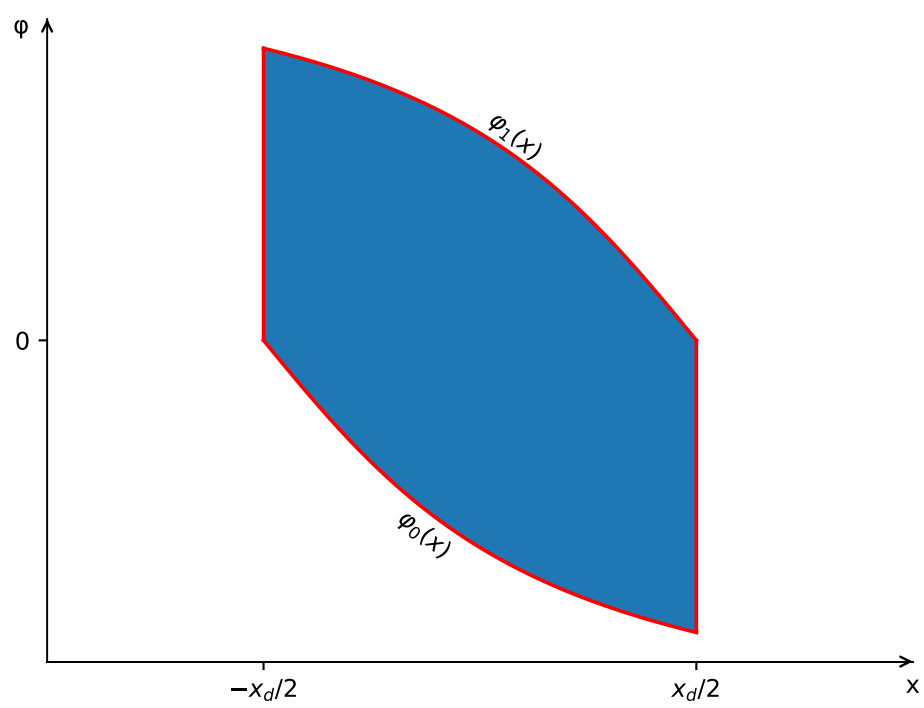

Figure 45. Acceptance shape in phase space.

The acceptance of this detector-collimator combination corresponds to the area of this surface, which in this case would be

$$
a=\left[2 \frac{x_{d}}{y_{d}} \tan ^{-1} \frac{x_{d}}{y_{d}}-\ln \left(1+\frac{x_{\mathrm{d}}^{2}}{y_{\mathrm{d}}^{2}}\right)\right] y d
$$


For small opening angle detectors, when $x_{d} / y_{d} \ll 1$ this reduces to

$$
a=\frac{x_{d}}{y_{d}} x_{d}
$$

which is just a product of the active area size $x_{d}$ and the opening angle, which is equal to $x_{d} / y_{d}$ in the small angle approximation. This approximation is valid within $1 \%$ relative error for opening angles up to ten degrees.

\subsection{Acceptance Reduction Due to a Magnetic Field}

As mentioned earlier if a magnetic field is present the geometric relations become considerably more complicated and the Monte Carlo method was used to study the acceptance with magnetic field. Trajectories inside the detector-collimator system were calculated using a tracking algorithm with low computational cost and extremely good long-term accuracy - the so-called Boris algorithm. It solves the discretized equation of motion in electromagnetic field through the relations

$$
\begin{gathered}
\frac{x_{k+1}-x_{k}}{\Delta t}=v_{k+1}, \\
\frac{v_{k+1}-v_{k}}{\Delta t}=\frac{q}{m}\left[E_{k}+\frac{\left(v_{k+1}+v_{k}\right) \times B_{k}}{2 c}\right] .
\end{gathered}
$$

Even though this algorithm is not symplectic, it conserves the phase space volume, which is an essential condition for a good particle tracking simulation. A more detailed description of this method and its advantages can be found in the paper by J. Boris [22].

Figure 46 shows that the angular range in the presence of a magnetic field is not affected much for points close to the middle of the detector active surface, but is considerably reduced for points close to the edges. One should expect that the acceptance decreases compared to the case with straight trajectories. 

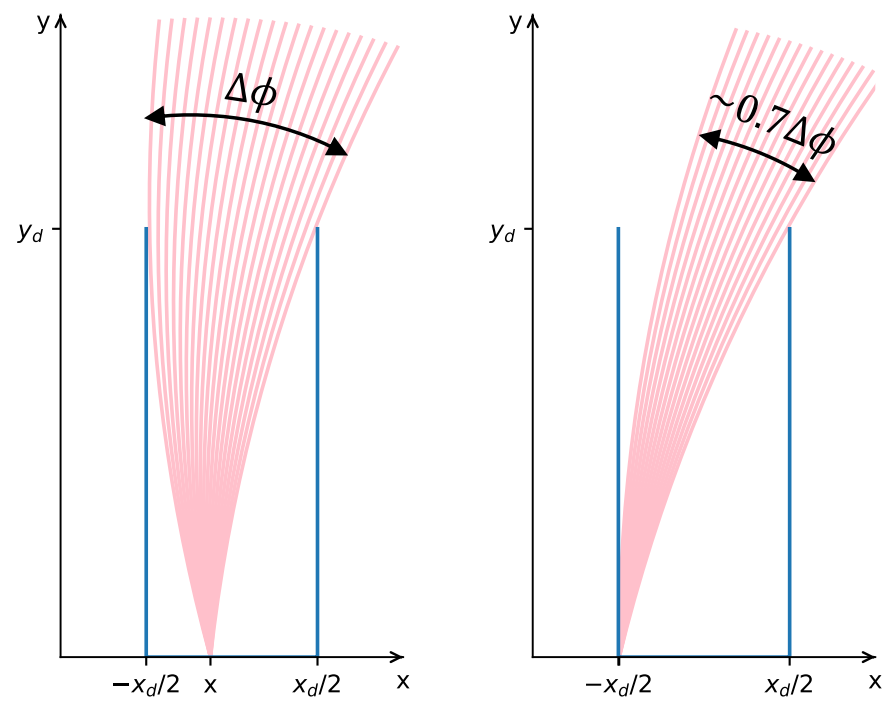

Figure 46. Acceptance reduction due to trajectories curvature.

To numerically calculate the acceptance of the detector in the presence of a magnetic field the Monte Carlo method can be utilized. One generates a set of random starting positions on the surface of the detector that are evenly distributed, followed by randomly selecting the initial angle of the trajectory within some angular range. The range can be chosen arbitrarily, from $-\pi / 2$ to $\pi / 2$ for example, the only requirement being that it must cover all possible good trajectories orientations. An optimal range would be $\left[-2 \tan ^{-1}\left(-x_{d} / 2 y_{d}\right), 2 \tan ^{-1}\left(-x_{d} / 2 y_{d}\right)\right]$ instead of $[-\pi / 2, \pi / 2]$ as it reduces the number of bad trajectories, thus saving computation time.

After tracking the trajectories and counting those that can leave the detector-collimator system (good trajectories), the value of the detector acceptance will be

$$
a_{m c}=4 x_{d} \tan ^{-1}\left(\frac{x_{d}}{2_{y d}}\right) \frac{N_{g o o d}}{N_{\text {total }}} .
$$

The relative error of this method is proportional to the inverse square root of the number 
of good trial samples. To check the accuracy of this Monte Carlo method the acceptance of the detector in the absence of a magnetic field was calculated analytically and compared to the Monte Carlo result. Trajectory sets used for comparison (25, 100 and 1000) are shown in Figure 47.
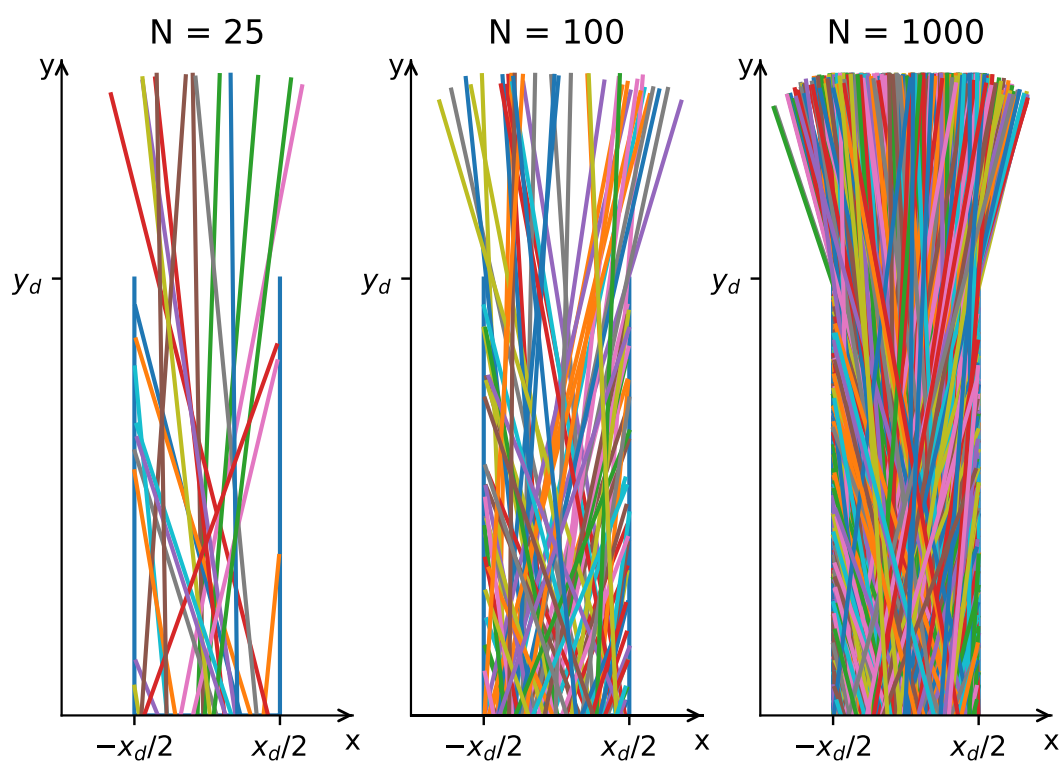

Figure 47. Monte Carlo acceptance calculation trajectories without magnetic field.

Results of the comparison are shown in Figure 48. On the $y$ axis the Monte Carlo calculated acceptance is plotted, normalized to the analytical value, versus the logarithm of the number of trial trajectories N. For $10^{4}$ trials the relative error is on the order of a few percent. Using the same method one can investigate the effect of the magnetic field presence on the detector acceptance. Using the Boris algorithm, one can now study the effect of the magnetic field on the acceptance for the 2-dimensional case as well as for the real detector-collimator configurations. 


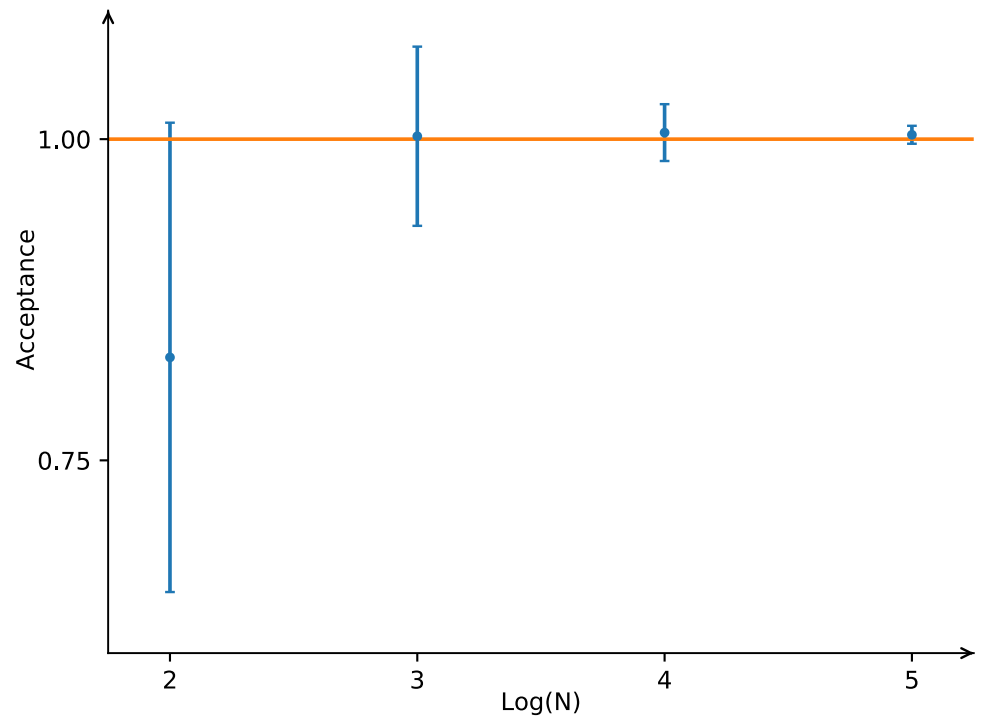

Figure 48. Monte Carlo calculated acceptance (normalized by theoretical value) with relative error.

The acceptance reduction strongly depends on the magnitude of the magnetic field, or to be more accurate, on the trajectory curvature angle on the length of the detectorcollimator system, which can be inferred form Figure 46. In the trajectories sets shown in Figure 49 this angle is about $15^{\circ}$, while the detector opening angle is about $20^{\circ}$. This rotation angle of the velocity vector is relatively high, for instance in the MAST magnetic field strengths the curvature of the trajectory on the length of the detector is about $2^{o}$ only, and detector opening around $6^{\circ}$. Despite of the relatively high trajectory curvature used in calculation, the difference between the calculated acceptance with a magnetic field compared to the acceptance without a field is on the order of $1 \%$, so that it can be neglected in rate calculations and the analytical acceptance value can be used. The same conclusion applies to the 3D case. 

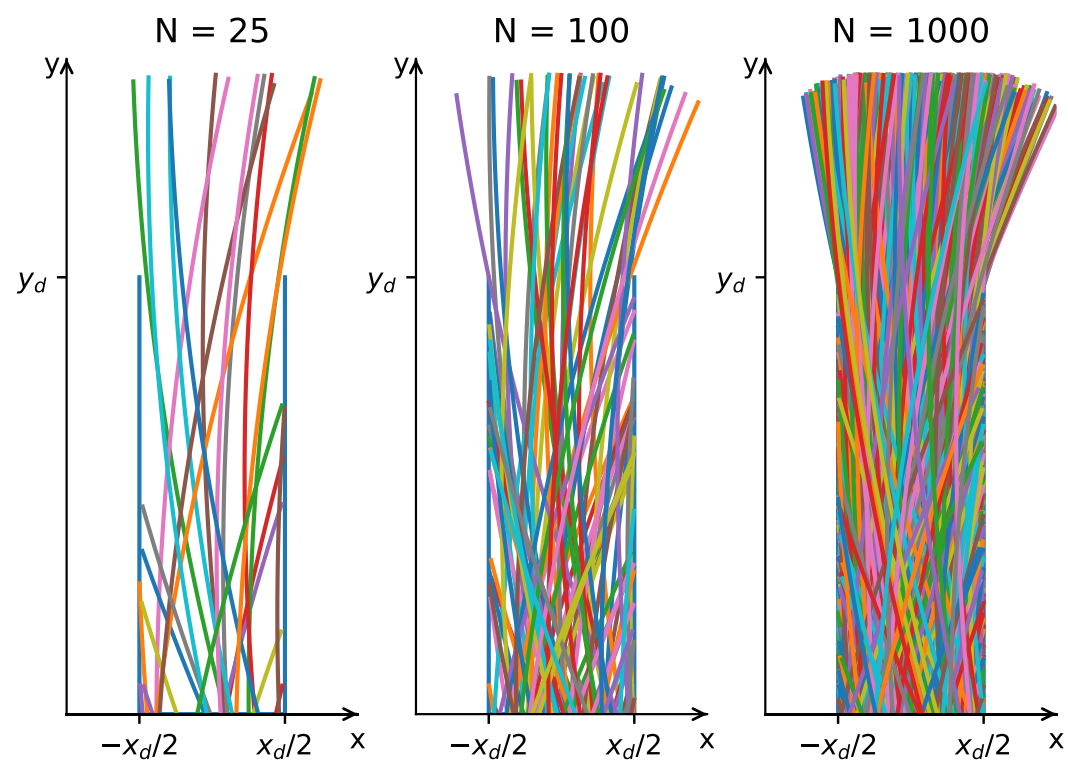

Figure 49. Monte Carlo acceptance calculation trajectories (2D) with magnetic field.

\subsection{Rate Calculation Based on TRANSP Modeled Emissivity Profile}

This section describes the calculation of particle rates measured by a detectorcollimator combination given an equilibrium magnetic field and an emissivity function. It is assumed that the emissivity is only a function of the poloidal position but not the fusionproduct angle. This corresponds to the case where the fusion reaction cross-section is isotropic. This is typically a good approximation, but anisotropy effects need to be analyzed and will be discussed in more details later.

To calculate the particle rate observed by a detector one needs to integrate the emissivity over the entire acceptance of the detector-collimator combination. In the two dimensional case, this integral looks is

$$
\dot{N}=\frac{1}{2 \Pi} \oint \xi(x, y) \Delta \varphi(x, y) d x d y
$$


where $\xi(x, y)$ is the emissivity profile containing the number of fusion products per unit time per unit area produced at the specified location and flying into equally probable directions ( $2 \mathrm{D}$ case). In other words, it can be written as a volume integral of the trajectories manifold in phase space (Figure 51) weighted by the emissivity, where the $\varphi$ dependence of the emissivity is applicable to the anisotropic reaction cross-section case

$$
\dot{N}=\frac{1}{2 \Pi} \int_{M} \xi(x, y, \varphi) d \Gamma .
$$

The problem is how to find $\Delta \varphi(x, y)$ at an arbitrary point of space. One needs to know the time reversed propagation of the detector phase space acceptance. The Monte Carlo simulated detector phase space shape is best for this study.

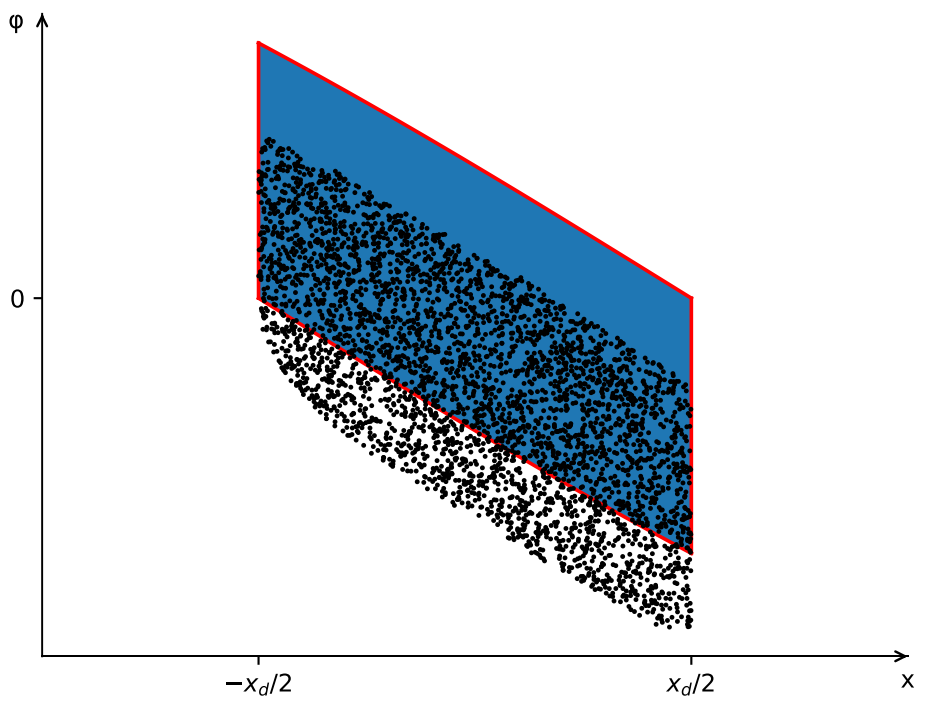

Figure 50. Monte Carlo simulated detector acceptance phase space points (black dots), and theoretical acceptance without magnetic field (blue parallelogram).

In Figure 50 the acceptance phase space shape is shown with black dots while the analytical 
calculation of the acceptance without a magnetic field is shown as a blue area parallelogram. It is noticeable that the magnetic field shifts the acceptance slightly.

Starting with the given initial positions and directions based on the simulated acceptance phase-space shape, one can track the particles and follow their trajectories in phase space rather than plotting $x-y$ projections only (Figure 51). The accepted angle range at any position, $\Delta \varphi \varphi(x x, y y)$, can be found numerically.

This approach will be necessary if the detector has a large angular acceptance and emissivity varies significantly over the manifold width. In our case the opening angle of the detector is small, and the emissivity can be assumed to be constant over the width of the manifold since the probing bundles are quite narrow. Consequently, the emissivity changes only along the central probing trajectory. In this approximation we need to integrate the cross-section of the manifold multiplied by the emissivity along the central probing trajectory to obtain the expected particle flow rate.

The remaining problem is how to find the manifold cross-section, which can be found using Liouville's theorem. Liouville's equation governs the time evolution of the phasespace density function $f f(p p, q q, t t)$ :

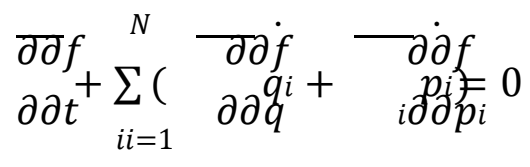

where $p$ and $q$ are the canonical momenta and coordinates.

Liouville's theorem states that the distribution function is constant along phase space trajectories. Another formulation is that the volume taken by a hypothetical ensemble in phase space stays constant in time,

$$
\int d \Gamma=\underset{\text { Vpphaasse }}{\int} d 3 p d 3 q=\text { coonnsttaanntt. }
$$



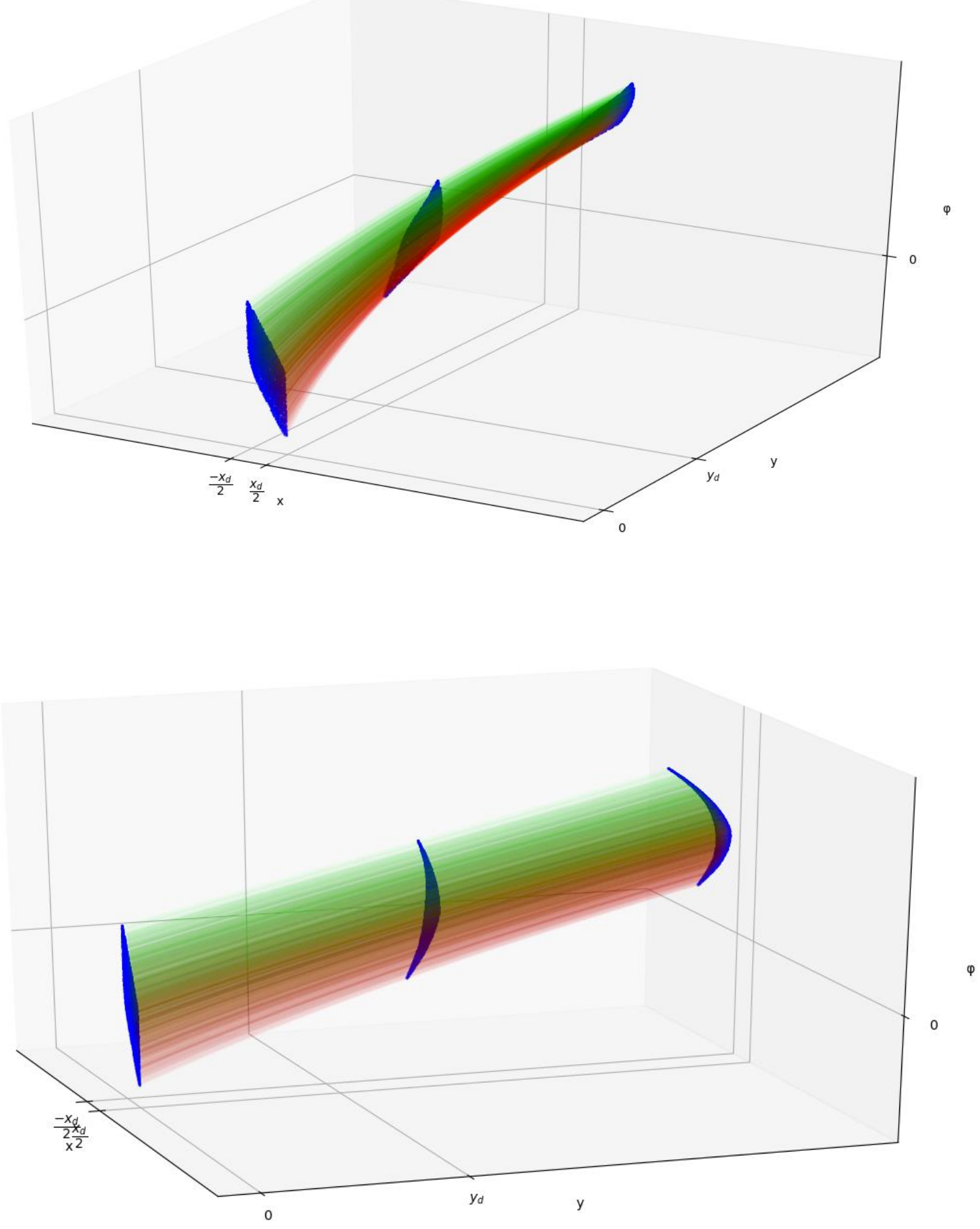

Figure 51. Phase space manifold of accepted trajectories showing the time evolution of the detector phase-space acceptance ensemble. 
For a small opening-angle detector the phase space manifold cross section will remain equal to the initial detector acceptance value, thus to calculate the expected rate we need to move along the bundle central probing trajectory and integrate the emissivity value along it, multiplied by the detector acceptance.

$$
\dot{N}=a \int \xi(l) d \vec{l}
$$

\subsection{Rate Calculation Approach Analytical Justification}

To prove the rate calculation method described by Equation 87 one can again consider a simplified case of 2D free-particle motion in the absence of a magnetic field. The detector active area of the size $x_{d}$ in a general case can have its normal $\vec{n}_{d}$ vector being tilted with respect to the sightline by some angle $\theta$, and letting the angular acceptance of the detector be $\Delta \theta$ as shown in Figure 52.

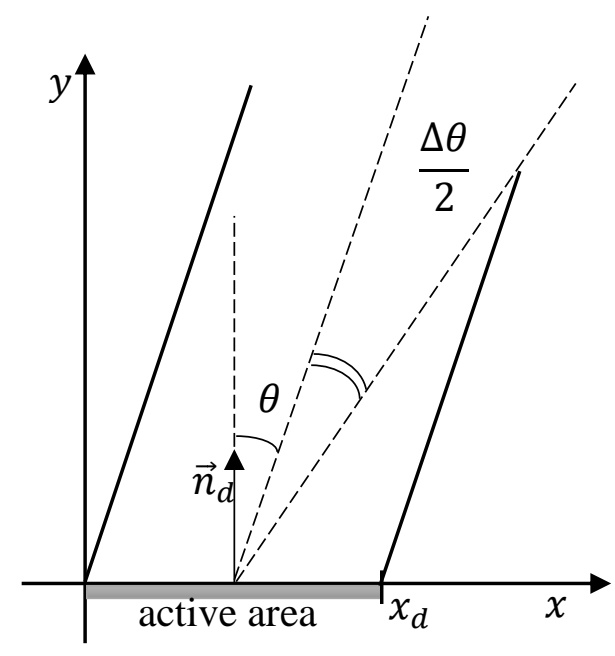

Figure 52. Tilted detector geometry.

If one knows the phase space density function $f(p, q, t)$ of the fusion products next to the active area, the particle flow per unit area per unit time could be calculated as

$$
\frac{d \dot{N}}{d s}(q, t)=\int_{p_{a c c}} \vec{v} \cdot \vec{n}_{d} f(p, q, t) d p
$$


where $\vec{v}$ is velocity vector, $\vec{v}=\left(\dot{q}_{x}, \dot{q}_{y}\right)$, and the integral is taken over all accepted particles with momenta defined by the opening angle and orientation of the detector.

The phase space density function satisfies the collisionless Boltzmann equation, which in the absence of forces that can change the particle momenta is

$$
\frac{\partial f}{\partial t}+\sum_{i=1}^{N}\left(\frac{\partial f}{\partial q_{i}} \dot{q}_{\imath}+\frac{\partial f}{\partial p_{i}} \dot{p}_{\iota}\right)=\mathrm{S}_{\mathrm{Em}} .
$$

On the right side of the equation is the source term due to the emission of fusion products, and can be expressed using the $2 \mathrm{D}$ emissivity given by

$$
S_{E m}(p, q)=\frac{\xi(\theta, q) \delta\left(p-p_{o}\right)}{p_{0}} .
$$

Here the emissivity $\xi$ is a function of the emission angle and position, corresponding to a general case of anisotropic reaction cross-section. When considering an isotropic reaction cross-section, the emissivity angle dependence disappears. The 1D Dirac delta-function indicates that the emitted particles are monoenergetic, $E_{0}=\frac{p_{0}^{2}}{2 m}$, which is a valid simplification despite the fact that the real fusion products have some finite energy spread due to the reaction kinematics, which will be discussed later.

The phase space density function can be considered time independent since the emissivity profile is quasi steady as the time scale of the emissivity changes is much larger than the time of flight of particles leaving the plasma

$$
\frac{\partial f}{\partial t}=0
$$

For free particles the Hamiltonian is

$$
H=\frac{\vec{p}^{2}}{2 m}
$$


and

$$
\dot{p}=-\frac{\partial H}{\partial q}=0 ; \quad \dot{q}=\frac{\partial H}{\partial q}=\frac{p}{m}
$$

The Boltzmann equation then becomes

$$
\frac{\partial f}{\partial x} p_{x}+\frac{\partial f}{\partial y} p_{y}=\frac{m}{p_{0}} \xi(x, y) \delta\left(p-p_{0}\right)
$$

The density function $f(p, q)$ should also be a delta function in $p$ :

$$
f(p, q)=f_{q}(q) \delta\left(p-p_{0}\right)
$$

Rewriting the momentum components in polar coordinates where the angle is measured as shown in Figure 52 and integrating over momentum magnitude $p$ we get

$$
\frac{\partial f_{q}(x, y)}{\partial x} \sin \theta+\frac{\partial f_{q}(x, y)}{\partial y} \cos \theta=\frac{m}{p_{0}^{2}} \xi(x, y) .
$$

Integrating this expression along the path of the central trajectory one finds:

$$
\begin{gathered}
\frac{\partial f_{q}(x, y)}{\partial x} \sin \theta d \vec{l}+\frac{\partial f_{q}(x, y)}{\partial y} \cos \theta d \vec{l}=\frac{m}{p_{0}^{2}} \xi(x, y) d \vec{l}, \\
\frac{\partial f_{q}(x, y)}{\partial x} d x+\frac{\partial f_{q}(x, y)}{\partial y} d y=\frac{m}{p_{0}^{2}} \xi(x, y) d \vec{l},
\end{gathered}
$$

and

$$
\int_{(x, 0)}^{\infty} \nabla f_{q} \cdot d \vec{l}=\frac{m}{p_{0}^{2}} \int_{(x, 0)}^{\infty} \xi(x, y) d \vec{l}
$$

From the fundamental theorem for line integrals and since $f(\infty)=0$,

$$
f_{q}(x, 0)=\frac{m}{p_{0}^{2}} \int_{(x, 0)}^{\infty} \xi(x, y) d \vec{l} .
$$

Using this result in Equation 88, and noting that $\vec{v} \cdot \vec{n}_{d}=\frac{p_{y}}{m}=\frac{p}{m} \cos \theta$, we get 


$$
\begin{aligned}
& \frac{d \dot{N}}{d x}(x)=\int_{p_{a c c}} \frac{p}{m} \cos \theta f_{q}(x) \delta\left(p-p_{0}\right) p d p d \theta= \\
& \theta+\frac{\Delta \theta}{2} \\
& \int_{\theta-\frac{\Delta \theta}{2}} \frac{p_{0}^{2}}{m} \cos \theta f_{q}(x) d \theta= \\
& \frac{p_{0}^{2}}{m} f_{q}(x)\left[\sin \left(\theta+\frac{\Delta \theta}{2}\right)-\sin \left(\theta-\frac{\Delta \theta}{2}\right)\right]= \\
& \frac{p_{0}^{2}}{m} f_{q}(x) \cos \theta 2 \sin \frac{\Delta \theta}{2}= \\
& \cos \theta 2 \sin \frac{\Delta \theta}{2} \int_{0}^{\infty} \xi(x, y) d \vec{l} \text {. }
\end{aligned}
$$

For a small opening angle $(\Delta \theta \ll 1)$, and considering the emissivity being constant on the width of the bundle, or $f_{q}(x, 0)$ being constant for $x$ on the detector surface size one gets

$$
\dot{N}=x_{d} \cos \theta \Delta \theta \int_{0}^{\infty} \xi(x, y) d \vec{l} .
$$

Since $x_{d} \cos \theta \Delta \theta=a$, where $a$ is a detector acceptance, this validates Equation 87 that can be used for the expected rate calculations based on the probing bundles and a given fusion emissivity profile extracted from the TRANSP simulations.

The validation of the above principle of rate calculation, which is based on Liouville's theorem of phase space volume conservation, would require more advanced considerations when the particles move in the presence of a magnetic field, since the canonical momentum would include the magnetic-field vector potential and the equations of motion will not be as simple as in free space. The detailed proof of the similar matter can be found in Application of Liouville's Theorem to Electron Orbits in the Earth's Magnetic Field by W. F. G. Swann [23]. 


\section{CHAPTER 5. PROTON ENERGY SPECTRUM}

The expected spectrum of protons emitted in a fusion reaction and detected by our diagnostic system is determined by multiple factors, such as the fusion reaction kinematics, the reaction cross-section angular dependence, and the probing trajectory location. As was stated earlier, most of the fusion reactions in experimental spherical tokamaks plasmas appear from the non-thermal neutral beam ions interacting with the thermal plasma ions. Because the energy of the fast neutral beam ions $(\sim 50 \mathrm{keV})$ is usually almost 100 times higher than the thermal ion energy, the reactions between them can be treated as a beamtarget interaction.

\subsection{Fusion Reaction Kinematics}

The geometry of the reaction kinematics for a stationary cold plasma ion hit by the energetic beam ion is shown in Figure 53. The velocity vector of a secondary fusion product is not shown to avoid the diagram overload.

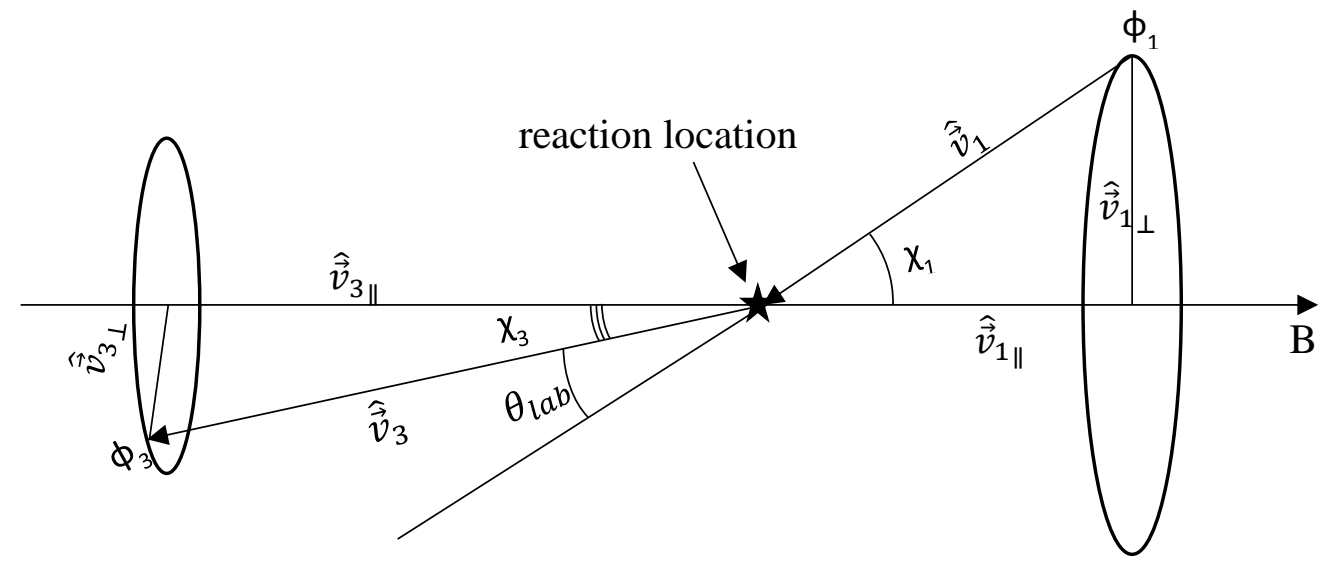

Figure 53. Reaction kinematics geometry (3D) depicting the fast beam ions (index 1) and fusion product (index 3) velocities unit vectors orientation relative to the magnetic field vector at the fusion reaction location. 
All the directions are specified relative to the magnetic field vector $\vec{B}$ at the reaction location. Index 1 denotes the projectile beam ion particle, which is a deuteron in our case, the variables with the index 2 correspond to the stationary plasma ion (also deuterons), indexes 3 and 4 are assigned to the outgoing fusion product particles, (proton and triton or neutron and ${ }^{3} \mathrm{He}$ for the $\mathrm{D}-\mathrm{D}$ reaction), $\chi$ denotes the pitch angle of the particle (the angle between particle's velocity vector and magnetic field vector), and $\phi$ is the gyro angle (a precession phase angle of a particle's velocity vector). Since we are detecting only one of the reaction products, velocity $\vec{v}_{4}$ is not shown on the kinematics diagram.

In the beam-target approximation, where $v_{1} \gg v_{2}$, target particle considered to be stationary at the reaction location and thus the vector $\vec{v}_{2}$ is not present on the reaction kinematics diagram. The center of mass velocity has almost the same direction as $\vec{v}_{1}$, so the angle of the outgoing particle 3 velocity $\theta_{\text {lab }}$ is the angle between velocity vectors $\vec{v}_{3}$ and $\vec{v}_{1}$.

The relation between the pitch angle of the fusion product velocity in the center of mass to that pitch angle in the lab frame can be found from momentum and energy conservation equations. Omitting the intermittent steps of the kinematics equations, the expression for this relation can be written as [20]

$$
\cos \theta_{c m}=-k_{0} \sin \theta_{l a b}+\cos \theta_{l a b} \sqrt{1-k_{0}^{2} \sin ^{2} \theta_{l a b}}
$$

where

$$
k_{0}=V \sqrt{\frac{m_{3}\left(m_{3}+m_{4}\right)}{2 m_{4}(Q+K)}}
$$


$m_{3}$ and $m_{4}$ are the fusion products masses, $V$ is the center of mass velocity, $Q$ is the reaction $Q$ factor defined in Capter 1 , and $K$ is the relative kinetic energy of the fusing particles.

\subsection{Cross-section Anisotropy}

In the center of mass frame, the cross-section of the $D(d, p) T$ reaction has a significant anisotropy. According to E. Brown and N. Jarmie [21], it can be parametrized in the form

$$
\sigma(\theta)=a+b \cos ^{2} \theta+c \cos ^{4} \theta,
$$

where sigma is a differential cross-section as a function of the center of mass angle $\theta_{c m}$. The asymmetry coefficients $b$ and $c$, as well as the total cross-section (implying the $a$ coefficient) depend on the collision energy. Further details can be found in [21]. The differential cross-section is shown in Figure 54 for an incident deuteron energy of $69.9 \mathrm{keV}$ which is close to the planned neutral-beam particle energy at MAST-U (75 keV).

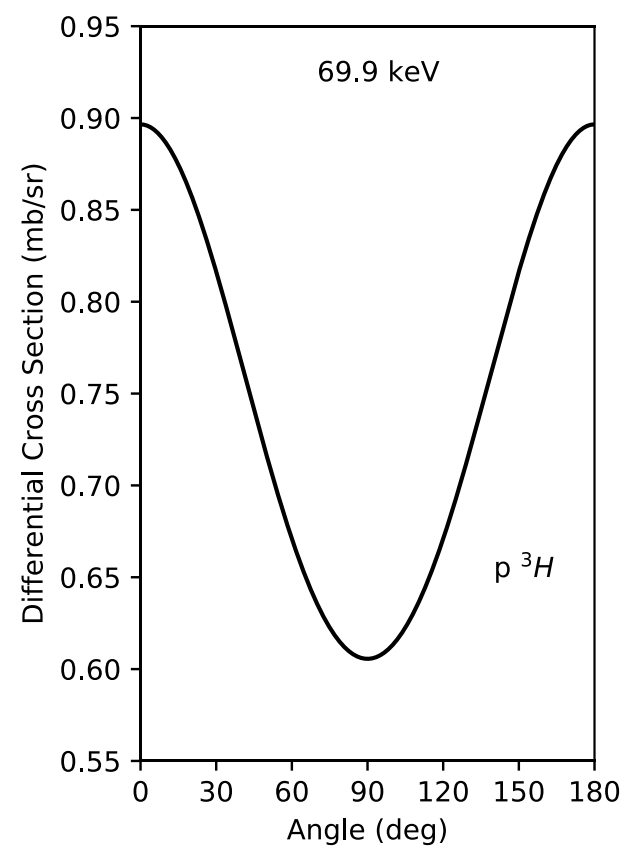

Figure 54. Differential cross-section of D(d, p)T fusion for incident energy of $69.9 \mathrm{KeV}$ as a function of center of mass angle [21]. 
The anisotropy is relatively strong, its minimum value is about $30 \%$ less than the maximum, so the fusion products have higher probability to have their velocity vectors being collinear to the incident beam particle direction. This anisotropy should be taken into account for the expected rate calculations.

The differential cross-section in the lab frame can be found from the one in the center of mass frame as

$$
\sigma_{c m}\left(\theta_{c m}\right) \sin \theta_{c m} d \theta_{c m}=\sigma_{l a b}\left(\theta_{l a b}\right) \sin \theta_{l a b} d \theta_{l a b}
$$

which implies the probability for reaction products scattering into a c.m. solid angle $d \Omega_{c m}=\sin \theta_{c m} d \theta_{c m} d \phi_{c m}$ is equal to the probability of scattering into the lab frame solid angle $d \Omega_{l a b}=\sin \theta_{l a b} d \theta_{l a b} d \phi_{l a b}$, and also uses the fact that $d \phi_{c m}=d \phi_{l a b}$.

Using the expression for $\cos \theta_{c m}$ (Equation 103), it is convenient to rearrange Equation 106 in the following way:

$$
\sigma_{l a b}\left(\theta_{l a b}\right)=-\frac{\sigma_{c m}\left(\theta_{c m}\right)}{\sin \theta_{l a b}} \frac{d \cos \theta_{c m}}{d \theta_{l a b}}
$$

Taking the derivative of $\cos \theta_{c m}$ with respect to $\theta_{l a b}$, one obtains the final expression for the differential cross-section in the laboratory frame to be

$$
\begin{gathered}
\sigma_{l a b}\left(\theta_{l a b}\right)=\sigma_{c m}\left(\arccos \left(-k_{0} \sin \theta_{l a b}+\cos \theta_{l a b} \sqrt{1-k_{0}^{2} \sin ^{2} \theta_{l a b}}\right)\right) \\
\cdot\left(\sqrt{1-k_{0}^{2} \sin ^{2} \theta_{l a b}}+\frac{k_{0}^{2} \cos ^{2} \theta_{l a b}}{\sqrt{1-k_{0}^{2} \sin ^{2} \theta_{l a b}}}+2 k_{0} \cos \theta_{l a b}\right)
\end{gathered}
$$

As can be seen from Figure 55 the transformation from the center of mass to the laboratory frame leads to a prevailing forward-direction scattering probability. The ratio between the total proton cross-section in the forward half $\left(0 \leq \theta_{l a b} \leq \pi / 2\right)$ : 


$$
\sigma_{f}=2 \pi \int_{0}^{\pi / 2} \sigma_{l a b}\left(\theta_{l a b}\right) \sin \theta_{l a b} d \theta_{l a b}
$$

and cross-section $\sigma_{b}$ in the region $\left(\pi / 2 \leq \theta_{l a b} \leq \pi\right)$ is $\sigma_{f} / \sigma_{b}=1.09$ in the case of the $\mathrm{D}(\mathrm{d}, \mathrm{p}) \mathrm{T}$ reaction of a $69.9 \mathrm{keV}$ deuteron beam and a target deuteron plasma ion.

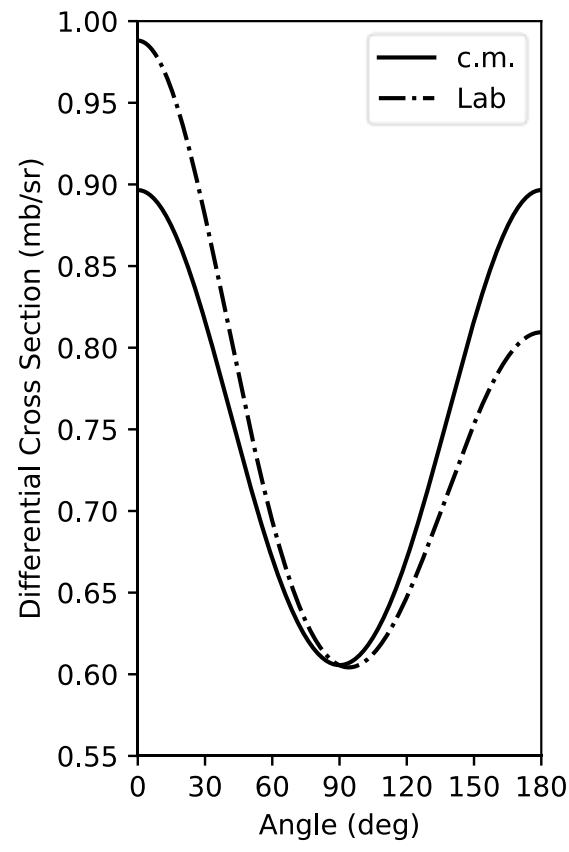

Figure 55. Differential cross-section in C.M. and laboratory frame. The differences are caused by transition from the moving C.M. to the laboratory frame.

One of the important questions related to the cross-section anisotropy is if it has a significant influence on the predicted rates. The modeled emissivity profiles contain the reaction rate per unit volume per unit time as a function of coordinates but assume that it is isotropic in a $4 \pi$ solid angle. Because of the cross-section asymmetry some directions may become more favorable and the emission rate per solid angle in a specified direction needs to be calculated instead. 
Considering the case of the anisotropic monoenergetic neutral beam with a given pitch angle $\chi_{1}$ at the location of the reaction and assuming the particles making their way into the detector have a pitch angle $\chi_{3}$, one can calculate the differential emissivity given by

$$
\frac{d \xi(\vec{r})}{d \Omega}=\frac{\xi_{4 \pi}(\vec{r})}{4 \pi} \frac{1}{\sigma_{\text {avg }}} \frac{1}{2 \pi} \int_{0}^{2 \pi} \sigma_{l a b}\left(\theta_{\text {lab }}\right) d \phi_{1},
$$

where $\xi_{4 \pi}$ is the integral emissivity in all solid angles given in the emissivity profile, and $\sigma_{\text {avg }}=\sigma_{\text {tot }} / 4 \pi$. The meaning of the integral is statistical averaging over the beam particle gyro angle $\phi_{1}$, which are evenly distributed in $2 \pi$.

Since the incident deuteron velocity vector and a fusion proton velocity vector are completely defined by their respective pitch and gyro angles, the relative angle between them can be found as

$$
\cos \theta_{l a b}=\cos \chi_{1} \cos \chi_{3}+\sin \chi_{1} \sin \chi_{3} \cos \phi_{1},
$$

which defines the $\theta_{l a b}$ as a function of $\phi_{1}$.

One can define the emissivity anisotropy scaling factor as

$$
k_{a n}\left(\chi_{1}, \chi_{3}\right)=\frac{1}{\sigma_{a v g}} \frac{1}{2 \pi} \int_{0}^{2 \pi} \sigma_{l a b}\left(\theta_{l a b}\right) d \phi_{1} .
$$

The predicted rate calculation then modifies to

$$
\dot{N}=a \int_{0}^{\infty} k_{a n} \xi(\vec{r}) d \vec{l} .
$$

One needs to keep in mind that since $k_{a n}$ depends on $\chi_{3}$, which is changing along the probing trajectory, thus it is a function of the reaction location $\vec{r}$. 
From Figure 56 one can infer that the change of the predicted rates after including the cross-section anisotropy can be expected to be on the order of up to 10 percent.

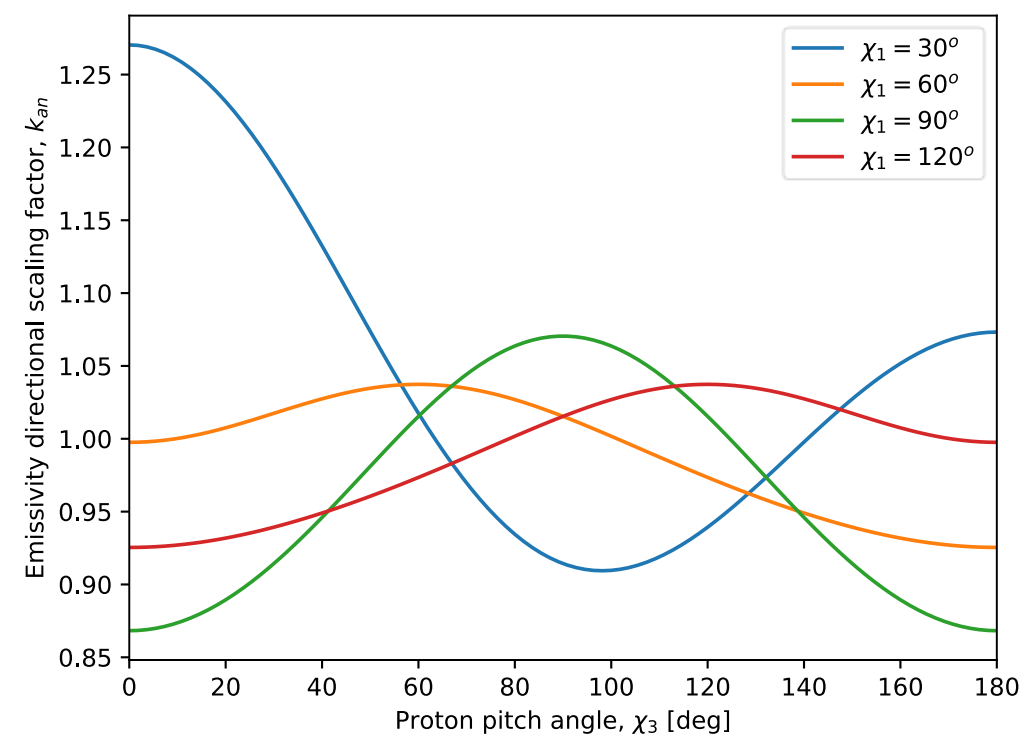

Figure 56. Emissivity anisotropy scaling factor as a function of the proton pitch angle $\chi_{3}$ for different pitch angles $\chi_{1}$ of a monoenergetic $69.9 \mathrm{keV}$ deuteron beam.

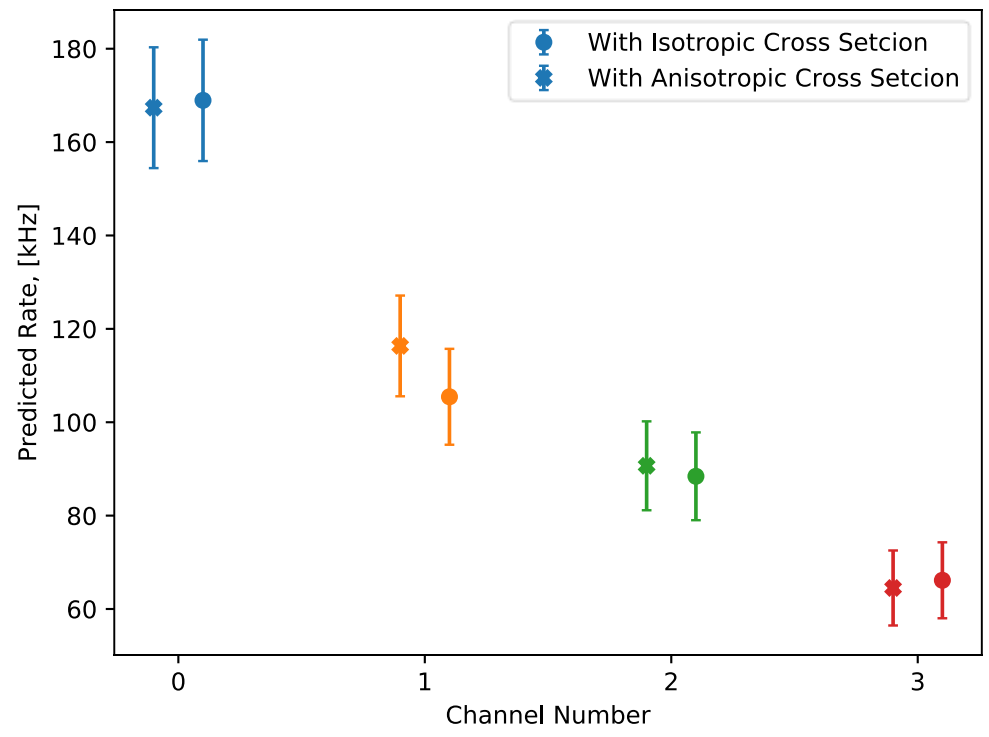

Figure 57. Cross-section anisotropy effect on predicted rates. 
The comparison of the predicted rates with and without the cross section anisotropy effect for the TRANSP emissivities is shown in Figure 57. The scaling factor $k_{a n}$ was calculated based on the proton pitch angle $\chi_{3}$ shown in Figure 67 and a beam pitch angle $\chi_{1}=\pi / 6$. The rate error bars are shown with an integration time of $\Delta t=1 \mathrm{~ms}$, which leads to a relative error of $10 \%$ for a rate of $100 \mathrm{kHz}(\delta R / R=1 / \sqrt{R \Delta t})$.

\subsection{Proton Spectrum Calculation}

According to [20], the energy of the fusion product with mass $m_{3}$ in the laboratory frame is given by the expression:

$$
E_{3}=\frac{m_{4}}{m_{3}+m_{4}}(Q+K)+V \cos \theta_{\mathrm{cm}} \sqrt{\frac{2 m_{3} m_{4}}{m_{3}+m_{4}}(Q+K)}+\frac{1}{2} m_{3} V^{2} .
$$

The Q-factor of the reaction causes a significant energy spread of the fusion protons. The proton energy in the center of mass frame does not depend on the scattering angle and for the $\mathrm{D}(\mathrm{d}, \mathrm{p}) \mathrm{T}$ reaction is $3.05 \mathrm{MeV}$ for an incident deuteron beam energy of $69.9 \mathrm{keV}$. In the lab frame, however, the proton energy varies from $2.7 \mathrm{MeV}$ to $3.4 \mathrm{MeV}$ (Figure 58).

For the proton spectrum calculation one can consider the reaction cross-section to be isotropic $\sigma_{c m}\left(\theta_{c m}\right)=$ const, which simplifies the problem of analytical spectrum derivation as done by W.W. Heidbrink [20]. In Monte Carlo calculations of proton spectral properties one can include the anisotropy from Equation 108.

From the proton energy (Equation 114) one can infer that the energy spectrum of protons emitted in all directions in the case of an isotropic cross section, will have a constant energy probability density function, since the energy depends linearly on $\cos \theta_{c m}$. 


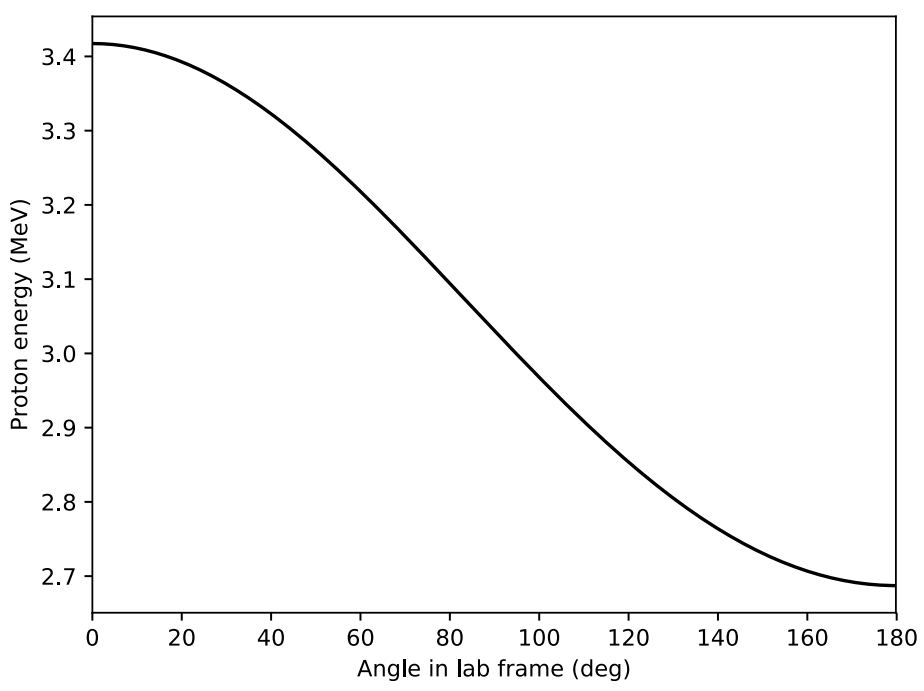

Figure 58. Proton energy vs scattering angle $\left(\theta_{l a b}\right)$ in the laboratory frame for the $\mathrm{D}(\mathrm{d}, \mathrm{p}) \mathrm{T}$ reaction at $69.9 \mathrm{keV}$ beam energy.

Note that a monoenergetic and unidirectional incident beam is implied and the particles are detected in a $4 \pi$ solid angle. The proton energy spectrum will be uniform for a fixed observation direction and an incoming beam having an isotropic direction distribution, what is referred to as a monoenergetic isotropic beam in [20].

To generate a set of incident velocity vectors with a random direction we can take a pitch angle $\chi_{1}=\cos ^{-1}(2 \operatorname{Rnd}(0,1)-1)$ and gyro angle $\phi_{1}=2 \pi \operatorname{Rnd}(0,1)$, where $\operatorname{Rnd}(0,1)$ is a random number from 0 to 1 with uniform distribution. Since for the reaction kinematics only the relative gyro angle is important, i.e. $\phi_{1}-\phi_{3}$, we can put $\phi_{3}=0$ without loss of generality.

Combining Equation 111 and Equation 103 one can calculate the scattering angle in the center of mass frame and calculate the proton energy using Equation 114. Taking the random sets for incident particle angles generated as described earlier the proton calculated energy $E_{3}$ can be histogrammed. 


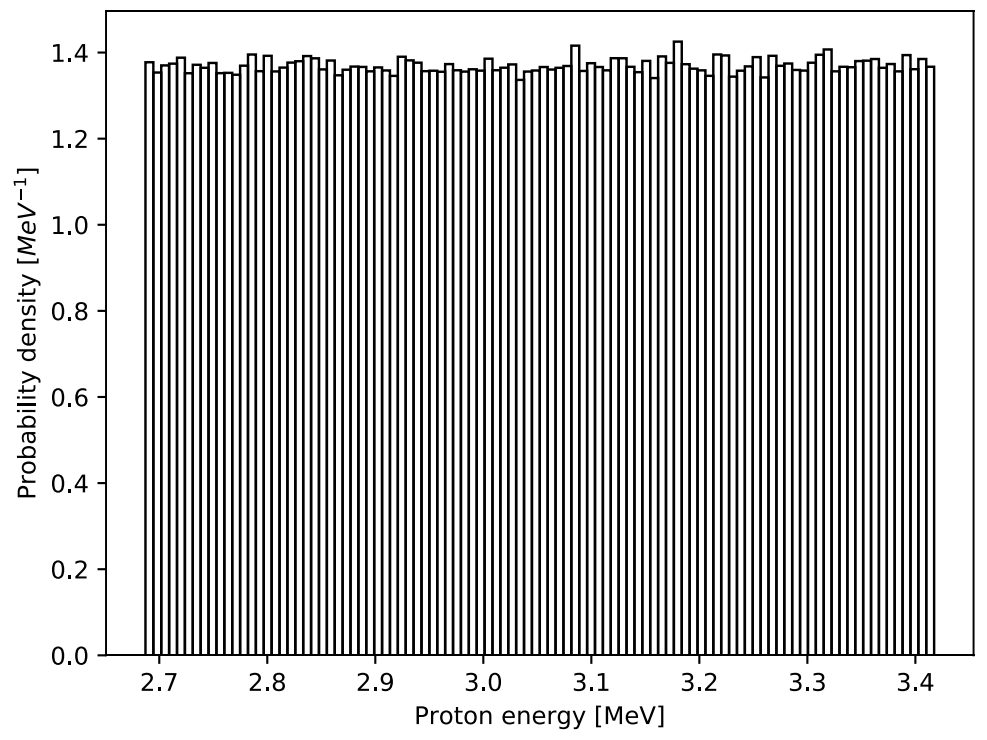

Figure 59. Proton spectrum from isotropic monoenergetic $(69.9 \mathrm{keV})$ beam fusion with target $(1 \mathrm{keV})$ plasma ions with isotropic cross-section (Monte Carlo simulation with $10^{6}$ samples).

Note that the energy range of resulting histogram (Figure 59) matches the one depicted in Figure 58. If the cross-section anisotropy is included in the calculation (Equation 105) the energy distribution becomes nonuniform, as shown in Figure 60.

In a real measurement the injected neutral beam ions have a pitch angle as well as an energy distribution, and consequently, the emitted fusion protons will have a more complex energy spectrum. To address these aspects one can start with the consideration of anisotropic monoenergetic neutral beam interacting with a thermal plasma and derive the produced spectrum of the emitted fusion products.

The expression for the energy distribution function of fusion products $F\left(E_{3}\right)$ for an isotropic cross-section can be found in [20]. Including angular dependence of the reaction cross section leads to the following new expression: 


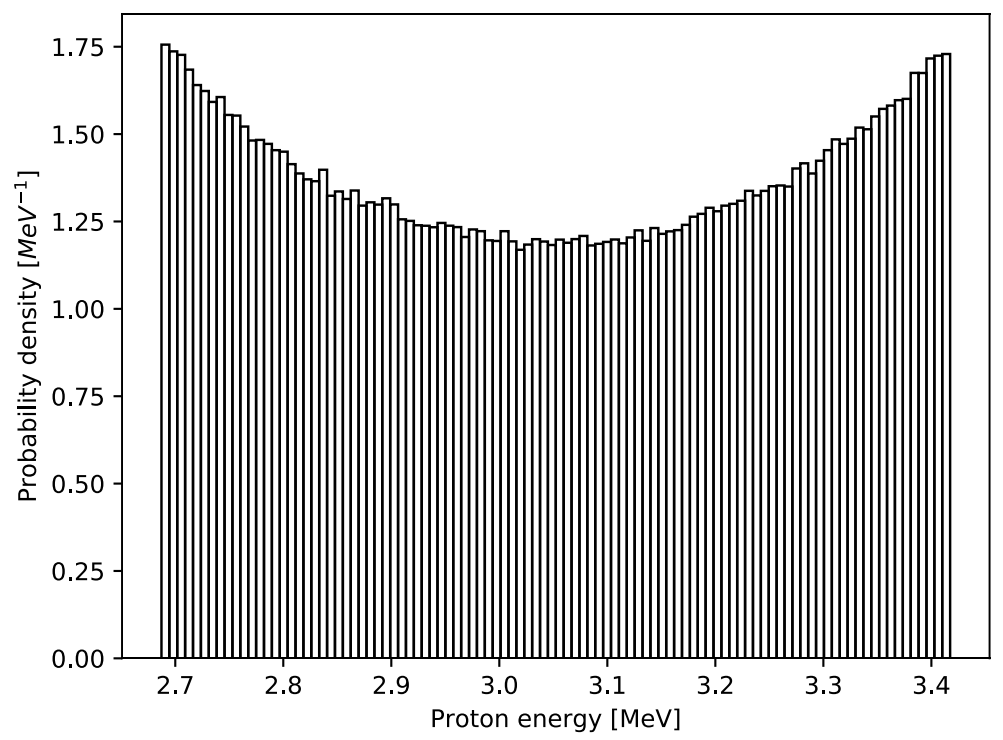

Figure 60. Proton spectrum from isotropic monoenergetic $(69.9 \mathrm{keV})$ beam fusion with target $(1 \mathrm{keV})$ plasma ions with anisotropic cross-section (Monte Carlo simulation with $10^{6}$ samples).

$$
\int_{E_{l}}^{E_{u}} F\left(E_{3}^{\prime}\right) d E_{3}^{\prime} \propto \int \sigma_{l a b}\left(\vec{v}_{1}\right) v_{1} f_{1}\left(\vec{v}_{1}\right) H\left(\vec{v}_{1}\right) d \vec{v}_{1},
$$

where $E_{l}$ is a lower energy limit of some arbitrary energy range, $E_{u}$ is an upper limit, and $H$ is a Heaviside type function defined as

$$
H\left(\vec{v}_{1}\right)=\left\{\begin{array}{rr}
1, & \text { if } E_{l}<E_{3}\left(\vec{v}_{1}\right)<E_{u} \\
0, & \text { otherwise }
\end{array}\right.
$$

For the anisotropic monoenergetic beam the probability distribution function is

$$
f\left(\vec{v}_{1}\right) \propto \delta\left(v-v_{b}\right) \delta\left(\chi-\chi_{1}\right)
$$

Equation 115 reduces to

$$
\int_{0}^{E} F\left(E_{3}^{\prime}\right) d E_{3}^{\prime} \propto \int_{\pi}^{\phi} \sigma_{l a b}\left(\phi_{1}\right) H\left(\phi_{1}\right) d \phi_{1} .
$$


Here we used the fact that the minimal energy corresponds to the gyro angle $\phi_{1}=\pi$ ( $\phi_{3}$ was agreed to be set equal to 0 ), since in this case the angle between incoming particle and fusion product is maximal and the energy $E_{3}$ is consequently minimal. One should keep in mind that the integral limits are interdependent, i.e. $E(\phi)$. Differentiating the right side of Equation 118 with respect to $E$ and the left side with respect to $\phi$ one gets

$$
F\left(E_{3}\right) \propto \sigma_{l a b}\left(\theta_{l a b}\right)\left(\frac{d E_{3}}{d \phi_{1}}\right)^{-1}
$$

Using Equation 114 one finds

$$
\frac{d E_{3}}{d \phi_{1}}=V \sqrt{\frac{2 m_{3} m_{4}}{m_{3}+m_{4}}(Q+K)} \frac{d \cos \theta_{c m}}{d \phi_{1}} .
$$

Since $\sigma_{c m}\left(\theta_{c m}\right) d \cos \theta_{c m}=\sigma_{l a b}\left(\theta_{l a b}\right) d \cos \theta_{l a b}$, then

$$
\frac{d \cos \theta_{c m}}{d \phi_{1}}=\frac{d \cos \theta_{l a b}}{d \phi_{1}} \frac{\sigma_{l a b}}{\sigma_{c m}}
$$

and using Equation 111 and differentiating it with respect to $\phi_{1}$ leads to

$$
F\left(E_{3}\right) \propto\left(\sin \chi_{1} \sin \chi_{3} \sin \phi_{1}\right)^{-1} \sigma_{c m}\left(\theta_{c m}\right) .
$$

This is the so-called reduced fusion-product distribution function. For an incident beam perpendicular to the magnetic field and the observation direction perpendicular to the field as well, the resulting spectrum is shown in Figure 61. The twin-lobed shape of the distribution is a consequence of the beam ion gyro motion, since the fast ions have uniform distribution in gyro angle $\phi_{1}$, and $d E_{3} \sim \sin \left(\theta_{c m}\right) d \phi_{1}$ (Equation 120), for c.m. angles $\theta_{c m}$ 
close to 0 or $\pi$ the probability density for $E_{3}$ goes to infinity, but of course remains integrable, which can be inferred from Equation 118.

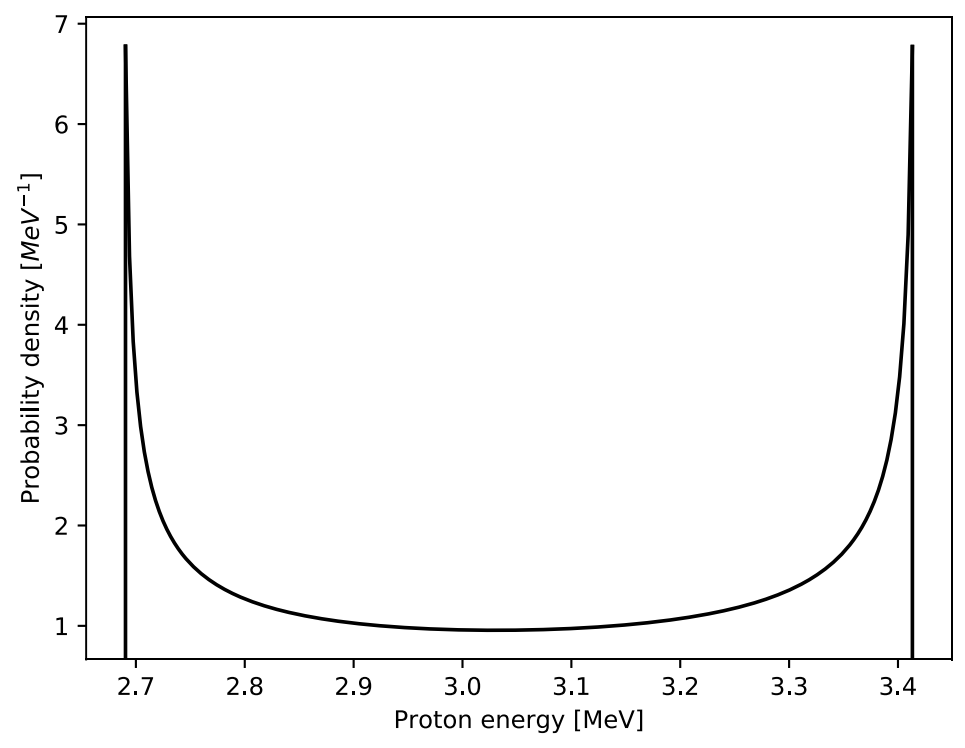

Figure 61. Proton spectrum emitted perpendicular to magnetic field $\left(\chi_{3}=\right.$ $\pi / 2)$ from anisotropic $\left(\chi_{1}=\pi / 2\right)$ monoenergetic $(69.9 \mathrm{keV})$ beam fusion with target (1 keV) plasma ions (isotropic cross-section assumed).

Convolving the reduced distribution function for all possible values $\chi_{3}$, taking into account that for an isotropic beam $\chi_{3}$ has a probability distribution of $\sim \sin \left(\chi_{3}\right)$, one can get the uniform proton energy distribution resulting in the same distribution function as in Figure 59. Due to the numerical convolution, the spectrum plot has spikes, which can be smoothed out but were left for demonstrative purpose to indicate that the obtained distribution indeed consists of the series of twin-lobed reduced distribution functions convolved together (Figure 62). 


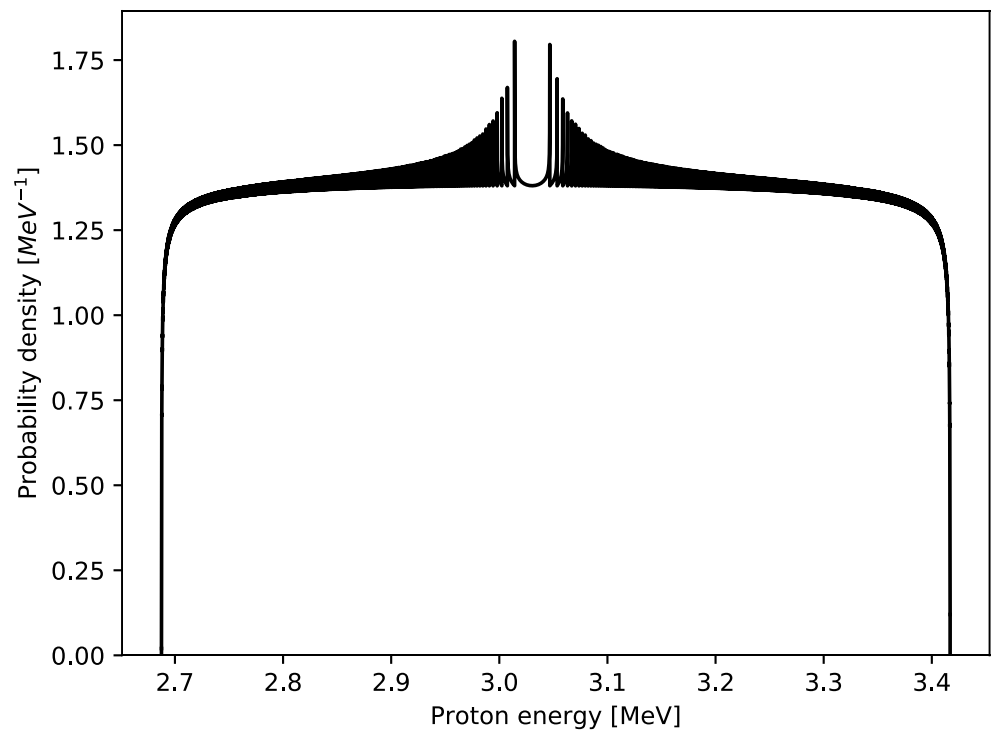

Figure 62. Proton spectrum emitted by isotropic monoenergetic $(69.9 \mathrm{keV})$ beam fusion with target $(1 \mathrm{keV})$ plasma ions with isotropic reaction crosssection (convolution of reduced distribution function).

For an anisotropic beam with energy distribution $f_{1}\left(E_{1}\right)$, the resulting proton energy distribution function can be found as an average of the reduced distribution functions with a weight $E_{1}$ (since in Equation $115 v_{1} d \vec{v}_{1} \sim v_{1} v_{1}^{2} d v_{1} \sim E_{1} d E_{1}$ ):

$$
F_{C}\left(E_{3}\right)=\int \sigma\left(E_{1}\right) E_{1} f_{1}\left(E_{1}\right) F\left(E_{3}, E_{1}\right) d E_{1}
$$

Using the result from Equation 122 for a reduced probability function, one can perform the convolution defined by the above equation for a given beam energy distribution and obtain a more realistic fusion-product energy spectrum. The following figures show the proton spectra for an anisotropic beam with thermal deuterium plasma ions, for a beam with a normal energy distribution around the maximum value of $69.9 \mathrm{keV}$ and a deviation of $17 \mathrm{keV}$, for various beam pitch angles $\chi_{1}$, and a proton pitch angle of $\chi_{3}=\pi / 3$. 
Figure 63Figure 64Figure 65Figure 66 contain the Monte Carlo simulated proton energy histograms which show the exact match with the analytically found spectra (analytically means using the reduced proton distribution function analytical expression and performing its numerical convolution).

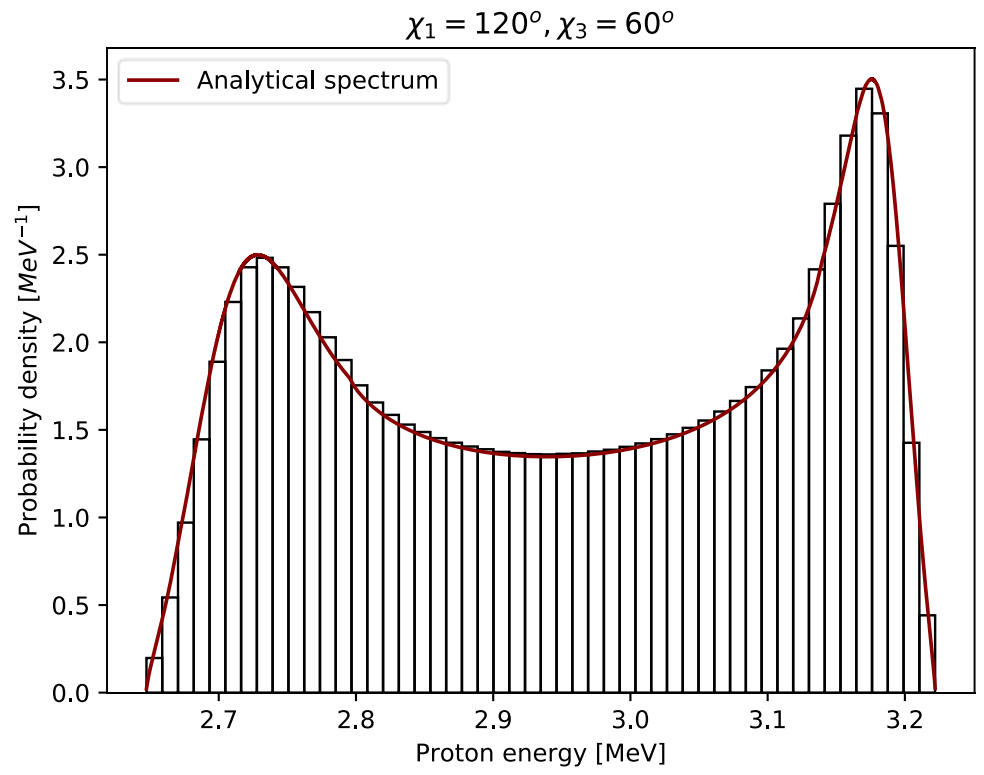

Figure 63. Analytical and Monte Carlo simulated proton spectrum emitted at $\chi_{3}=\pi / 3$ pitch angle, from fusion with anisotropic deuteron beam of pitch angle $\chi_{1}=2 \pi / 3$ and energy distribution $f(E) \propto \exp (-1 / 2$. $\left.(E-69.9 \mathrm{keV})^{2} /(17 \mathrm{keV})^{2}\right)$. 


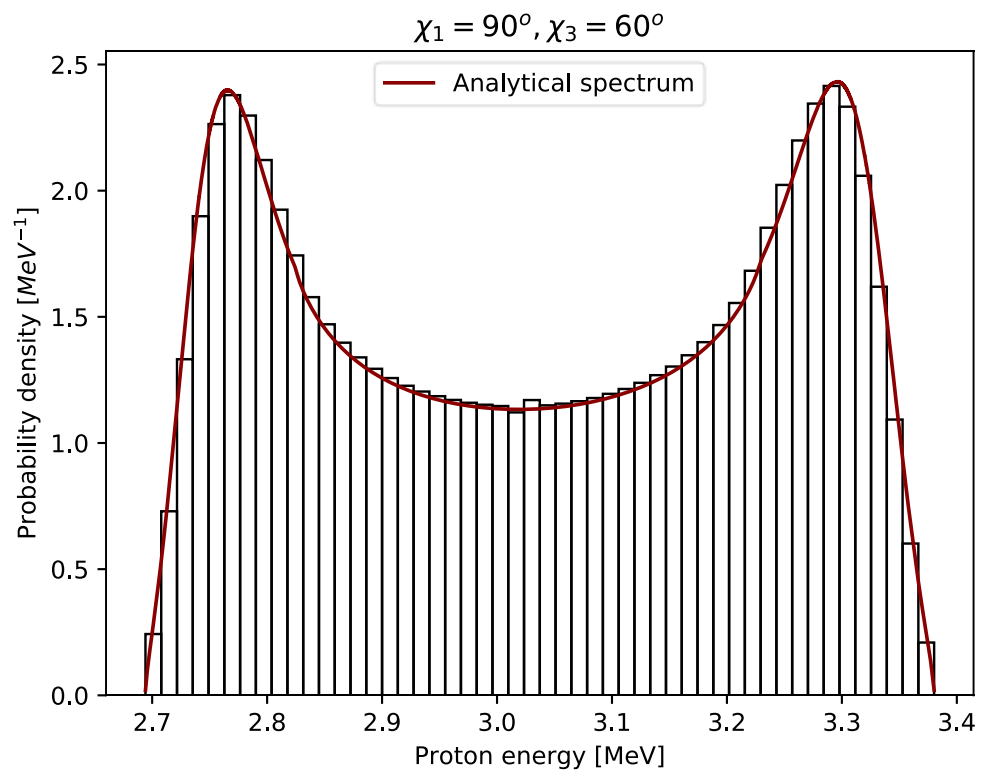

Figure 64. Analytical and Monte Carlo simulated proton spectrum emitted at $\chi_{3}=\pi / 3$ pitch angle, from fusion with anisotropic deuteron beam of pitch angle $\chi_{1}=\pi / 2$ and energy distribution $f(E) \propto \exp (-1 / 2$. $\left.(E-69.9 \mathrm{keV})^{2} /(17 \mathrm{keV})^{2}\right)$.

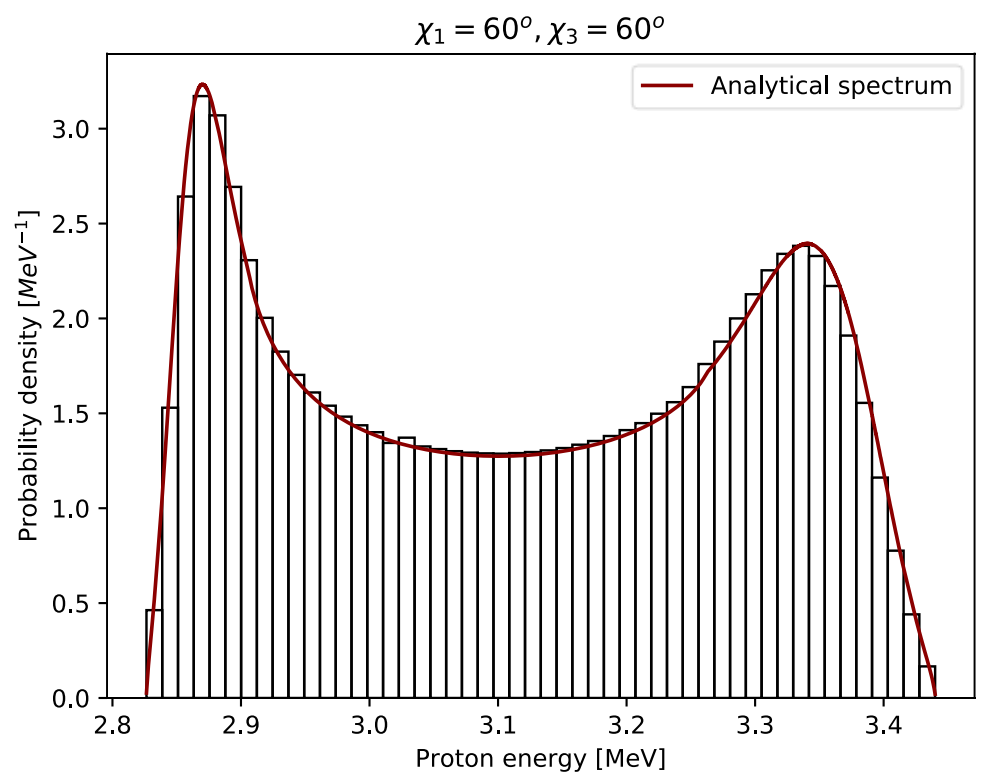

Figure 65. Analytical and Monte Carlo simulated proton spectrum emitted at $\chi_{3}=\pi / 3$ pitch angle, deuteron beam pitch angle $\chi_{1}=\pi / 3$ and energy distribution $f(E) \propto \exp \left(-1 / 2 \cdot(E-69.9 \mathrm{keV})^{2} /(17 \mathrm{keV})^{2}\right)$. 


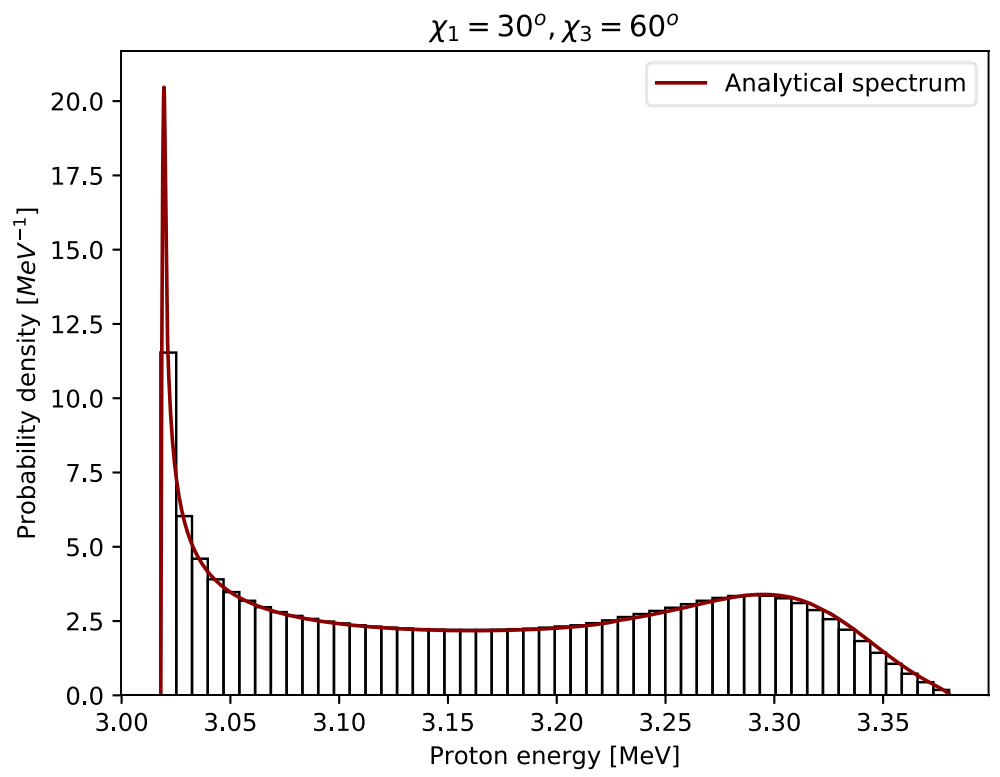

Figure 66. Analytical and Monte Carlo simulated proton spectrum emitted at $\chi_{3}=\pi / 3$ pitch angle, from fusion with anisotropic deuteron beam of pitch angle $\chi_{1}=\pi / 6$ and energy distribution $f(E) \propto \exp (-1 / 2$. $\left.(E-69.9 \mathrm{keV})^{2} /(17 \mathrm{keV})^{2}\right)$.

\subsection{Pitch Angle Variation Along the Probing Trajectory}

Up to this point we considered the spectrum of protons emitted either in all directions, which appears to be uniform, or in a single direction defined by the pitch angle $\chi_{3}$. In our diagnostic, each detector observes protons that are produced in a fusion reaction at some point along the trajectory bundle corresponding to the detector orientation and collimator openings. Since our bundles are relatively thin, for the spectral property calculation we use only the central trajectory for each detector. One can realize that the pitch angle is varying along the trajectory (Figure 67), thus the spectrum of protons detected by our system's individual channel will represent a convolution of the reduced distribution function 
described earlier with respect to $\chi_{3}$ along the probing trajectory with the weight factor equal to the fusion emissivity at each point.

The pitch angle variation along a central trajectory is shown in Figure 67. The energy spectrum for three different neutral beam pitch angles $\chi_{1}$ is shown in Figure 68. The fast neutral beam is not unidirectional but has some distribution in pitch angle $\chi_{1}$. This distribution can be obtained from TRANSP and an additional convolution can be carried out for obtaining more realistic predicted spectra. The measured spectral information thus can potentially be used to infer the fast ions distribution properties, which would require a deconvolution procedure that is expected to be difficult especially in the presence of the noise in the data and energy resolution limitations.

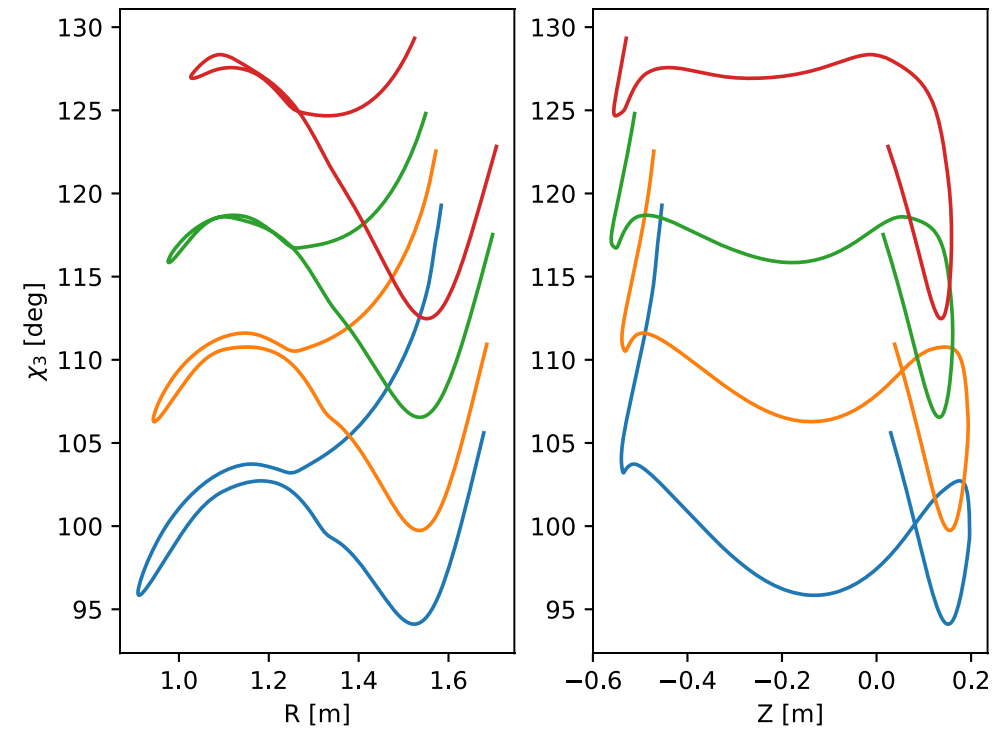

Figure 67. Pitch angle of the central probing trajectory as a function of radial and vertical position along the central probing trajectory for 4 detectors. 


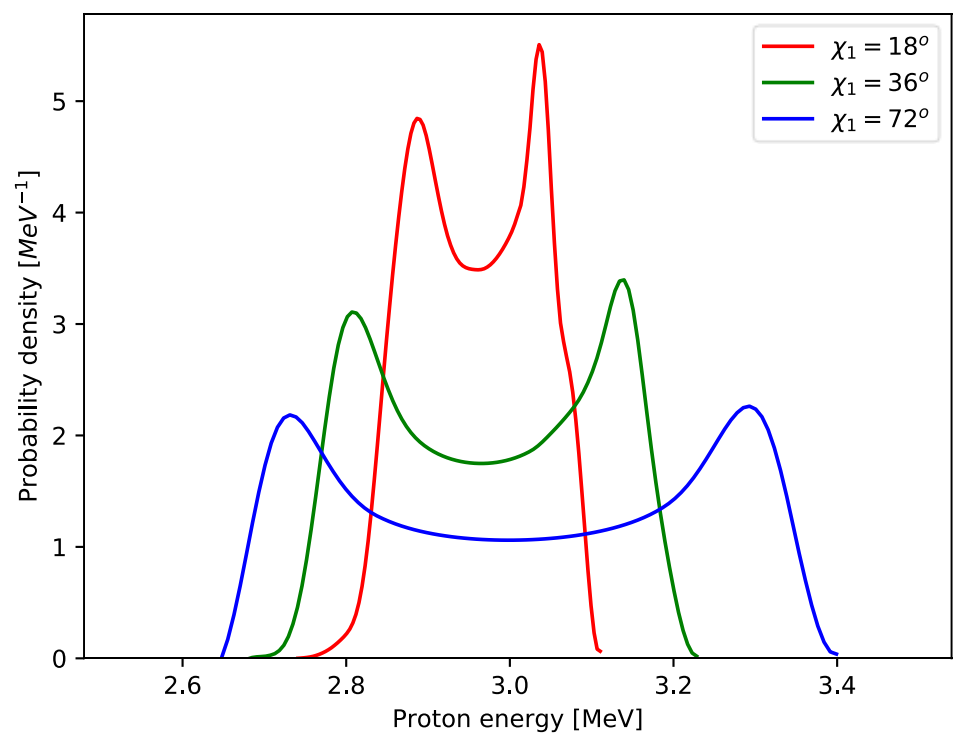

Figure 68. Expected proton spectrum emitted from thermal ions fusion with anisotropic deuteron beam of pitch angle $\chi_{1}$ and energy distribution $f(E) \propto$ $\exp \left(-1 / 2 \cdot(E-69.9 \mathrm{keV})^{2} /(17 \mathrm{keV})^{2}\right)$ detected by the channel with given pitch angle $\chi_{3}$ variation along the central probing trajectory.

For the proton diagnostic system tested in MAST 2013 experimental campaign, electrical noise introduced a substantial proton energy resolution deterioration that prevented the extraction of useful spectral characteristics, but the future resolution improvement attempts may help to obtain clearer and more informative proton energy spectra. 


\section{CHAPTER 6. DATA ACQUISITON AND ANALYSIS}

The data acquisition system for our detector probe consists of the signal processing chain of preamplifier, timing filter amplifier, digitizer and PC. The current pulse signals produced by the surface barrier detectors are integrated in Canberra 2003BT PreAmplifiers, which generate output signals with typical amplitudes of tens of millivolts for $\mathrm{MeV}$ particles and signal decay times of a few hundred microseconds. Those signals are then amplified by Ortec 474 and Canberra 2111 timing filter amplifiers with adjustable integration and differentiation times. The output signals have amplitudes of about $0.7 \mathrm{~V}$ and pulse widths of a few hundred nanoseconds. The amplified and shaped signals are then digitized using a GaGe Dynamic Signals Octopus CompuScope Express (CSE 8387) 16 (2×8) channel $100 \mathrm{MS} / \mathrm{s}$ digitizer with 14 bit resolution and 4G sample storage ( $2 \times 2 \mathrm{GS})$, and sent to the PC through a fiber optic link (Figure 69). The entire data acquisition system is controlled using a LabVIEW based program. The system is expected to achieve an energy resolution of around $150 \mathrm{keV}$ (FWHM) but the real life energy resolution can be strongly affected by electrical noise due to the high sensitivity of the detector system and the hostile electrical environment close to a tokamak plasma.

\subsection{Predecessor Diagnostic System}

A previous version of a similar system was tested in a MAST (Mega Ampere Spherical Tokamak) experiment carried out at the Culham Center for Fusion Energy in Oxford, UK. That system had four detectors and a different mechanical design. A typical example of raw data from MAST is shown in Figure 70. 


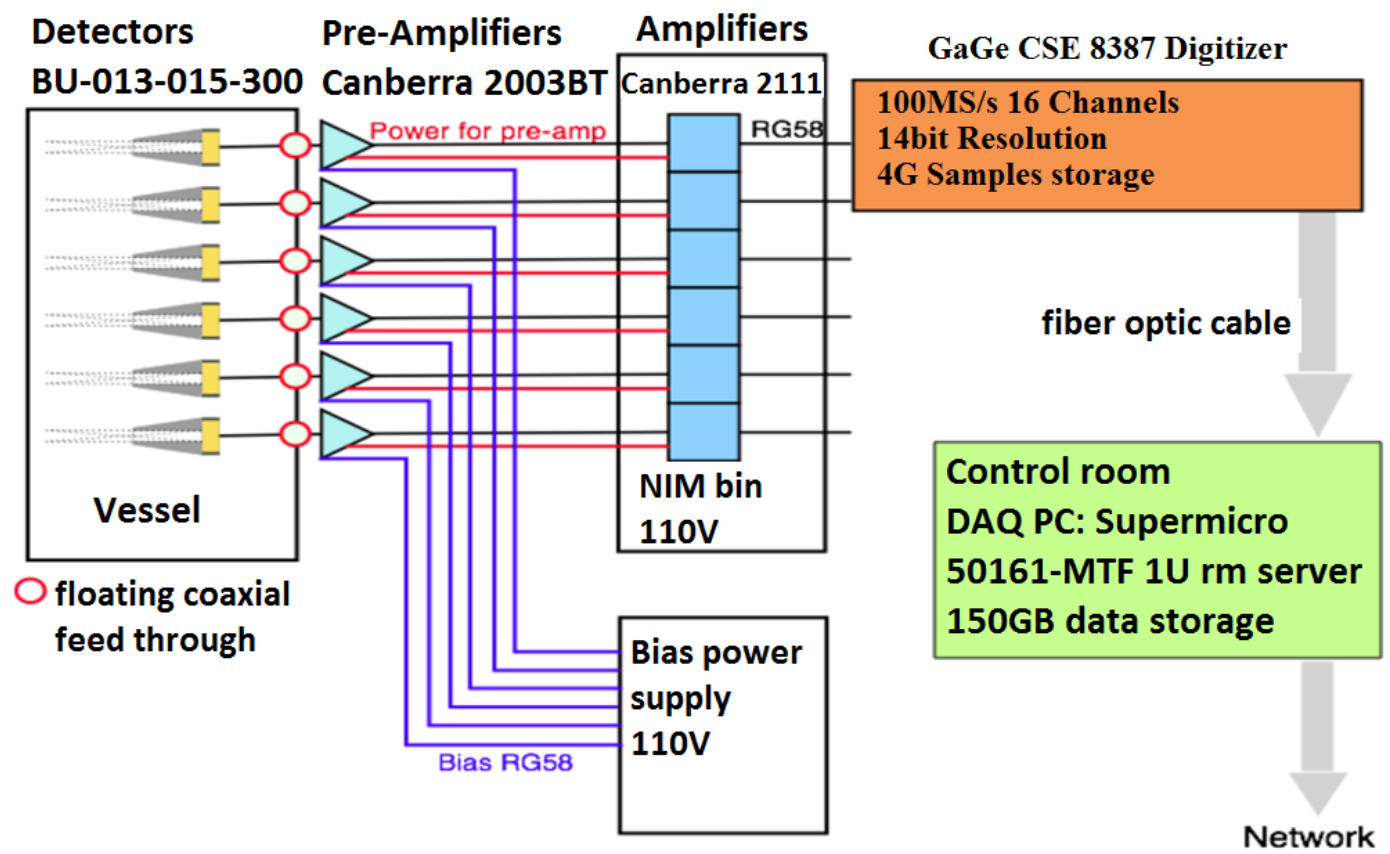

Figure 69. Data acquisition electronics scheme [19].

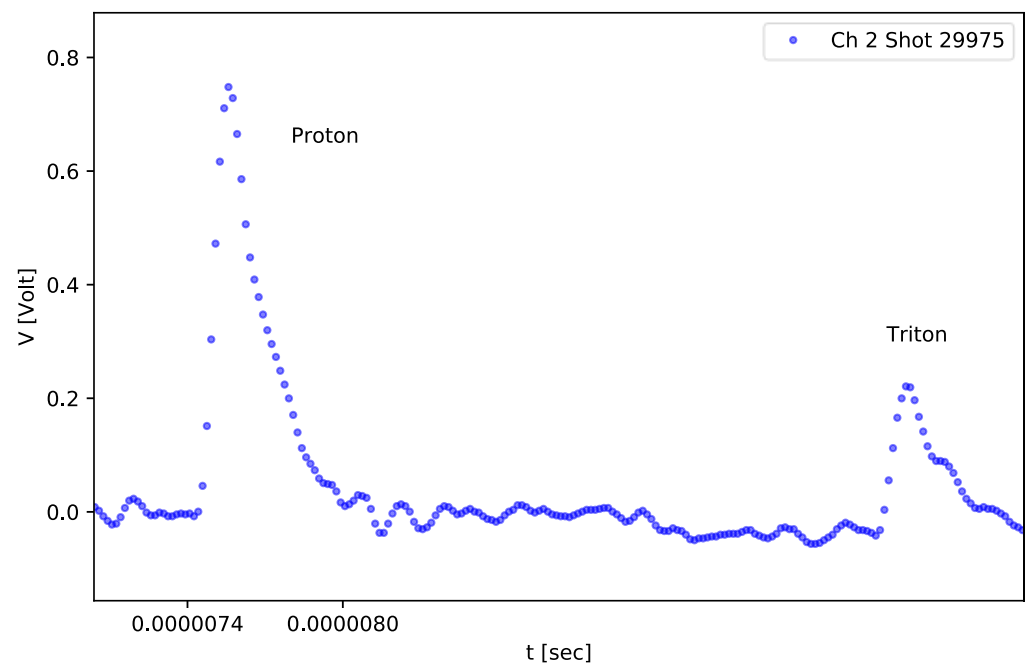

Figure 70. Typical signal shape from MAST data. The proton peak amplitude is $\sim 0.7 \mathrm{~V}$ and the triton peak is $\sim 0.23 \mathrm{~V}(3 \mathrm{MeV}$ and $1 \mathrm{MeV}$ energy particles), the peak width is of the order of $100 \mathrm{~ns}$.

The MAST 2013 experimental campaign demonstrated that this diagnostic system can work in a spherical tokamak. The new system has more channels (up to 8) and will be operated in plasmas with a considerably higher neutron rates. It is also more flexible as the 
collimator configuration can be changed for each detector. The online data acquisition program has been updated, which resulted in considerable speed gains for saving the acquired data.

\subsection{Data Analysis}

The raw (digitizer) data analysis procedure consist of three main steps: characteristic peak shape determination, raw data fitting, particle determination and rate calculation. For the first step a set of high quality particle signals need to be selected, which had to be done previously by visually inspecting the peaks and manually selecting those with a good shape. A new automatic selection process has been developed where a random set of high quality peaks are selected. The selection process starts with a selection of peaks with signals above a user defined threshold. These selected peaks are then normalized and an analytic expression for the peak shape is fit to the sample peaks. Only those peaks with a reduced chi square value below another user defined value are retained. A second fit is then performed where all selected and normalized peaks are fit with a common set of parameters. These define the peak shapes used in a full analysis of the raw data. The peak shape is given by the analytical

$$
V(t)=V_{0} e^{-c_{1}\left(t+t_{0}\right)}\left(t+\tanh c_{2}\left(t+t_{0}\right)\right),
$$

where $V_{0}$ is a normalization constant, $c_{1}^{-1}$ is a decay time, $c_{2}^{-1}$ is a rise time and $t_{0}$ is a constant related to $c_{1}$ and $c_{2}$ to bring the peak maximum to $t=0\left(V^{\prime}(0)=0\right)$. Typically, twelve peaks are used for the characteristic shape determination. An example of an automatic peak shape fit is shown in Figure 70. 


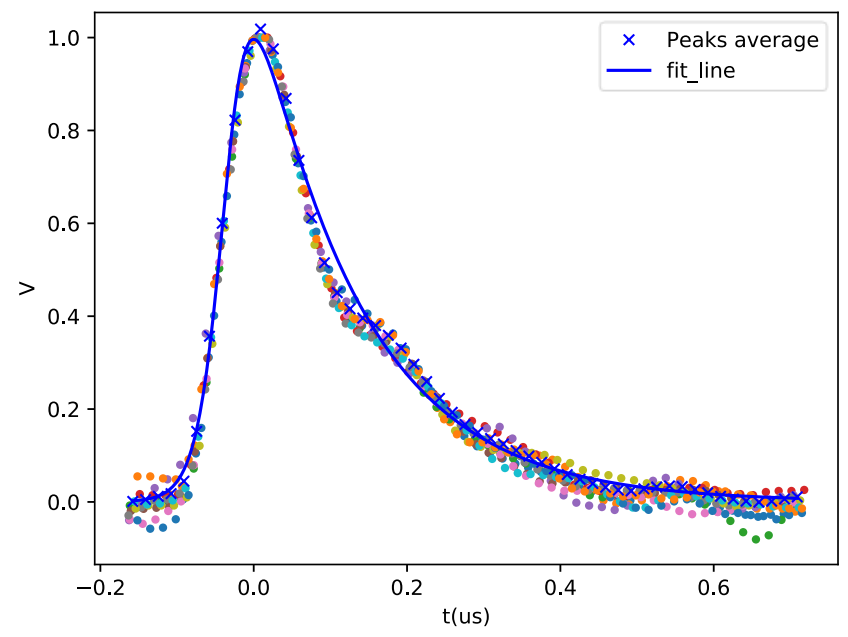

Figure 71. Superposition of all selected normalized peak data (points) and the common normalized peak shape.

Once the peak shape is determined, the raw data can be completely analyzed. As before the signal peak positions are determined and then the data are analyzed as described in reference [19].

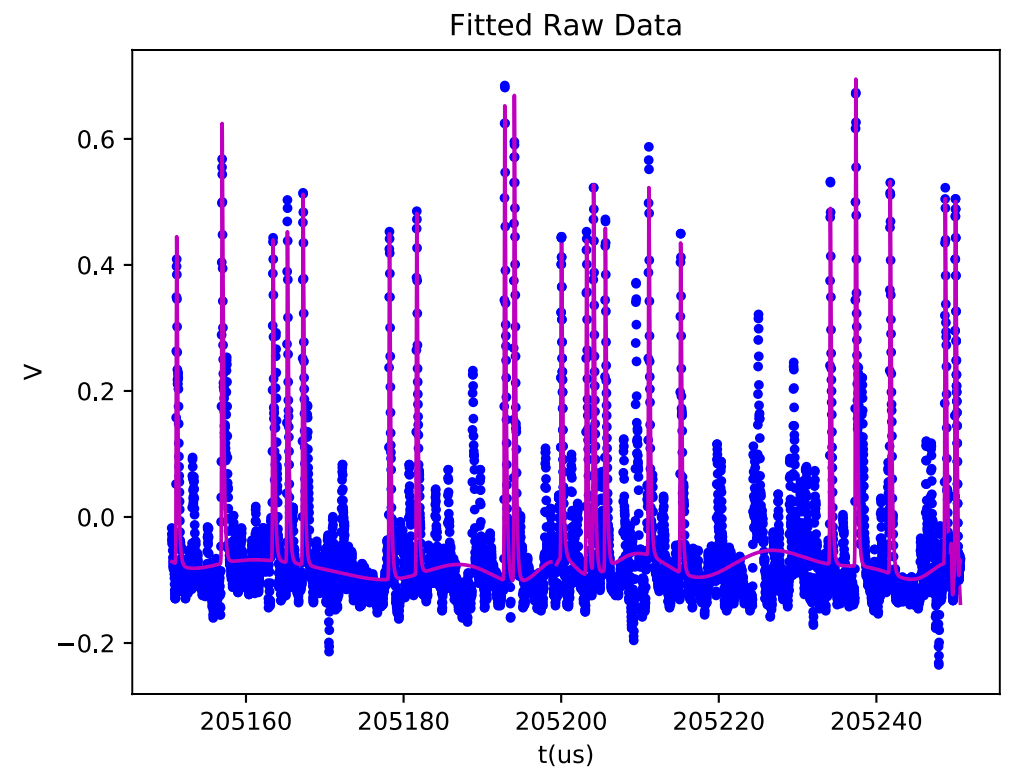

Figure 72. Subset of fitted data. A 10th order polynomial has been used as to model the background and the signal baseline. 
After fitting is complete, the resulting peak heights are analyzed providing time dependent energy spectra where protons and tritons can be identified (the ${ }^{3} \mathrm{He}$ peaks are hard to separate from the background). Selecting an integration time, the number of events within a selected energy range and time window are evaluated and the corresponding particle rate is calculated. A typical example of the particle rates as a function of time is shown in Figure 73. Details of the data analysis can be found in [19]. 
Shot : $29975 /$ Channels: $1,2,3$

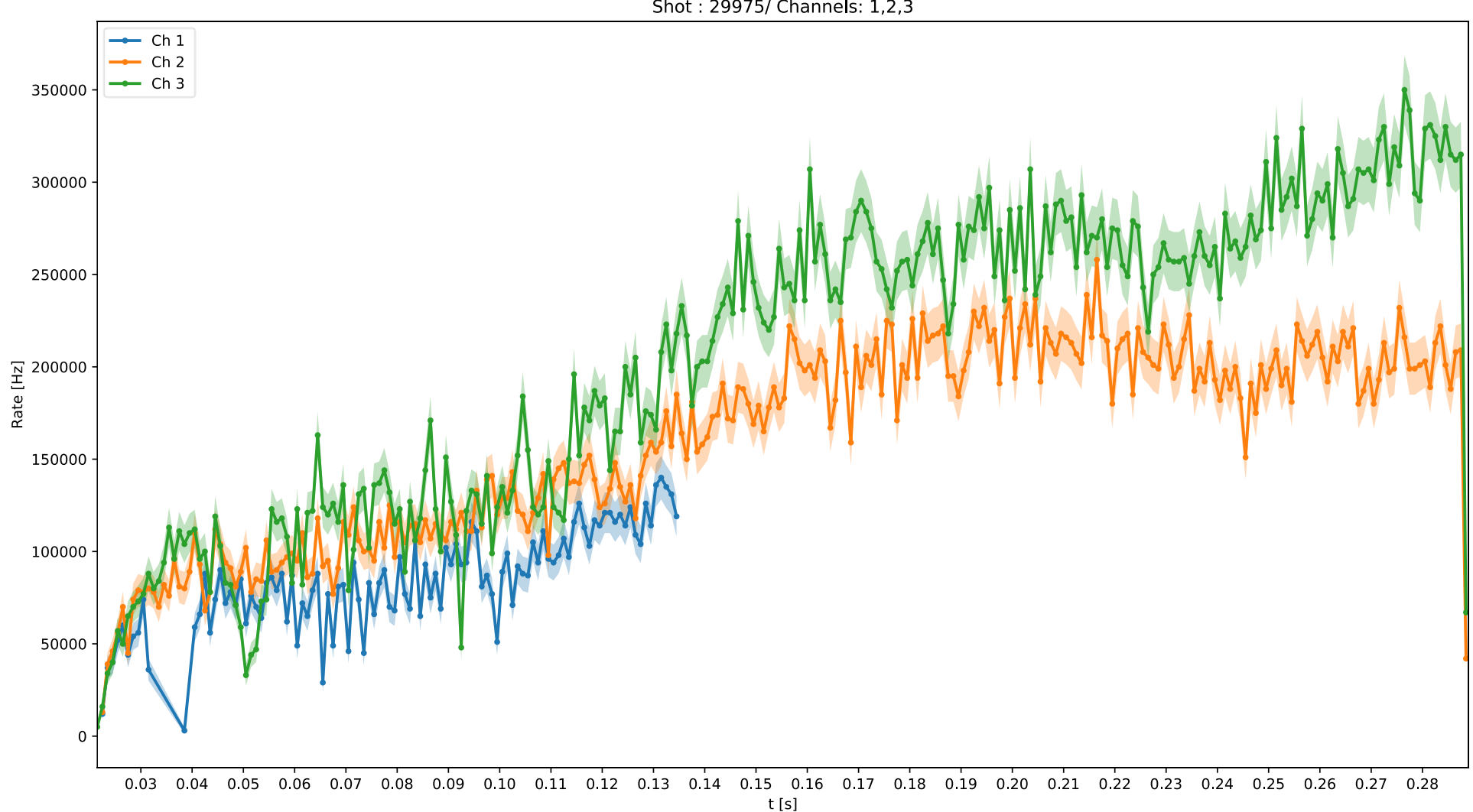

Figure 73. Experimental rates for MAST 2013 shot 29975 (1 ms integration). Data for three channels (three detectors) are shown with the shaded area around each curve being a statistical uncertainty. 


\subsection{Electrical Noise Testing}

One of the biggest obstacles to achieve good energy resolution of the detected particles is the presence of electrical noise in the raw data. The installation of the new diagnostic at the NSTX-U facility was planned for June 2016 but got canceled due to the discovery of a short in one of the poloidal field coils located on the center stack. As a consequence, the experimental campaign at NSTX-U had to be stopped and the machine disassembled. We were however able, using a preliminary installation of the existing DAQ to perform tests while NSTX-U was being started. This allowed us to perform early electrical noise testing with some detectors placed in the vicinity of the tokamak vacuum vessel.

High amplitude electrical noise, typically originating from switching power supplies, was detected with distinct spectral features shown in Figure 74. $250 \mathrm{kHz}$ noise pulses with an internal $3 \mathrm{MHz}$ structure are prominent in the test data. Electrical shielding of the electronic equipment, cable twisting and high frequency noise filters will be used, if necessary, to suppress this noise. Charged particles, probably produced by neutrons in the detectors, were also identified. The observed rates were significantly lower than those expected for protons $(\sim 100 \mathrm{~Hz}$ vs. $\sim 100 \mathrm{kHz})$ and are not expected to cause problems for proton identification. 


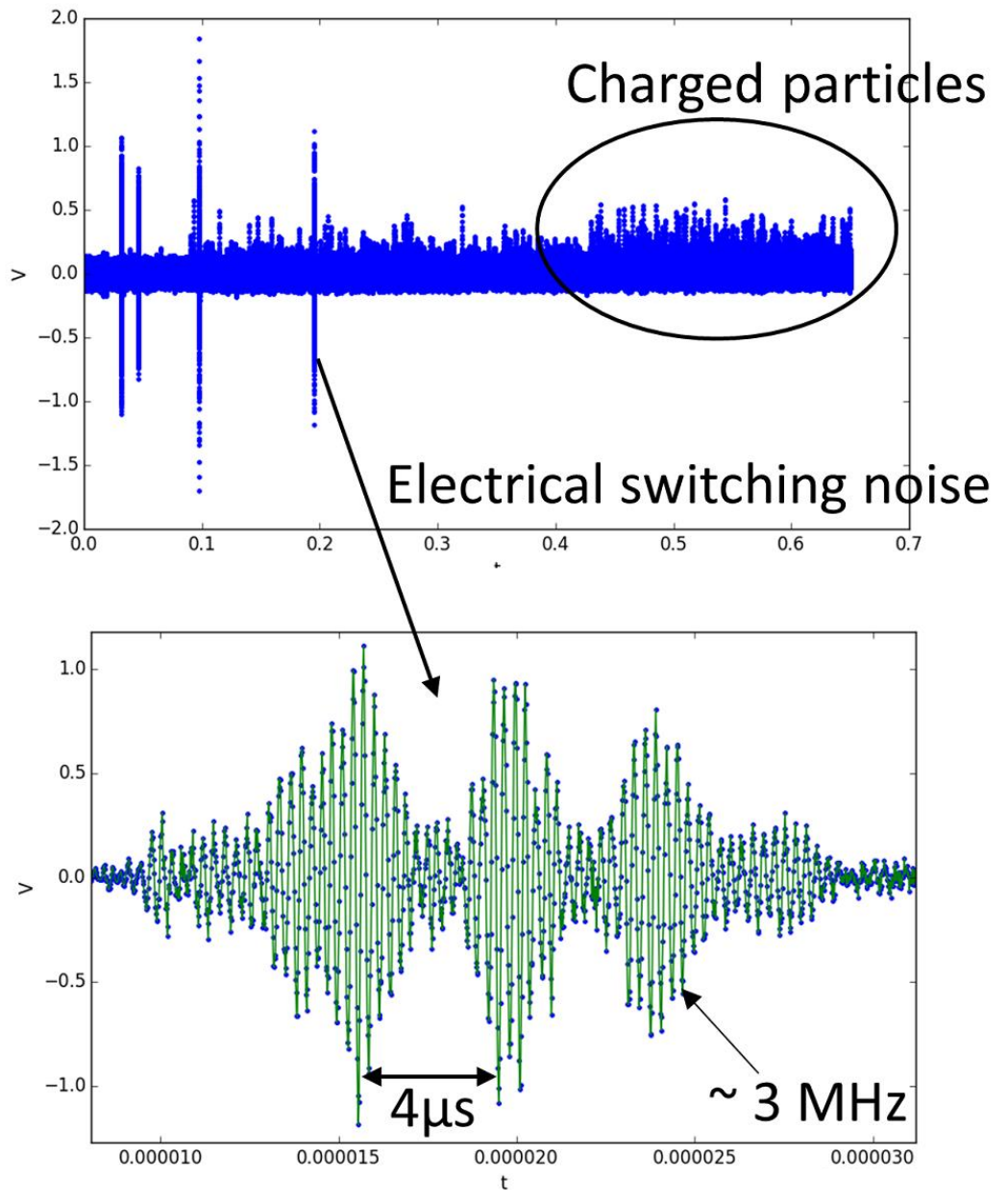

Figure 74. NSTX-U electrical noise testing results. 


\section{CHAPTER 7. TRANSP SIMULATIONS}

One of the goals of the diagnostic is a validation of the theoretically calculated emissivity profile, done by comparing the measured and expected particle detection rates. A neutron camera that is available at MAST-U detects the neutron flow rate through collimated sightlines that are straight versus the curved paths of the proton diagnostic, thus providing similar information as the proton system. Both systems provide complementary information on the emissivity profile and can be used in a combined analysis or to cross check the results from each instrument. The calculation of the expected rates requires two main inputs - the plasma equilibrium magnetic field to calculate the probing trajectories bundles, and the emissivity profile. The equilibrium magnetic field is calculated by the EFIT module as discussed in Chapter 2, and the emissivity profile calculated by the plasma particle and energy transport code TRANSP [27], [28].

\subsection{TRANSP Physics Overview}

TRANSP is a simulation code developed at PPPL for plasma particles, energy, and momentum time dependent transport. It assumes toroidal symmetry of the machine and utilizes a 2D coordinate system, where one coordinate is a normalized flux surface and the second one is the poloidal angle with respect to the magnetic axis. This set of coordinates have certain advantages for the solution of the transport equation, and the calculated quantities can be easily presented in standard poloidal cross-section coordinates $R$ and $Z$ afterwards.

TRANSP numerically solves the set of transport equations ([5], [16]), the particle balance equation has the form 


$$
\frac{\partial n}{\partial t}+\nabla(n \vec{u})=S
$$

where $n$ is the density of a given particle species, $\vec{u}$ is the average velocity of these particles, $S$ is a source or sink term that can be nonzero if the fusion reactions occur and particles are created or destroyed, or if neutral particles from neutral beam injection, pellets, or thermal neutrals are ionized.

The momentum balance equation has the following form:

$$
m n\left[\frac{\partial}{\partial t}+\vec{u} \cdot \nabla\right] \vec{u}=-\nabla \cdot \bar{P}+q n(\vec{E}+\vec{u} \times \vec{B})-S \vec{u}+\vec{A}
$$

where $m$ is the mass of a given particle species, $\bar{P}$ is the pressure tensor, $q$ is the charge and $\vec{A}$ is a term including the collisional momentum transfer due to forces like neutral beam thermalization drag, or ionization and recombination forces.

The energy balance equation can be written as

$$
\frac{3 \partial p}{2 \partial t}+\frac{3}{2}(\vec{u} \cdot \nabla) p+\frac{3 p}{2} \nabla \cdot \vec{u}+(\bar{P} \cdot \nabla) \cdot \vec{u}+\nabla \cdot \vec{q}_{h}=M-\vec{u} \cdot \vec{A}+\frac{1}{2} u^{2} S,
$$

where $\vec{q}_{h}$ is the heat flow vector and $M$ is the energy density change rate from interspecies collisions. The transport equations are reformulated in a diffusion like equation, with the general form

$$
\frac{\partial a}{\partial t}=\nabla \cdot\left(D_{a} \nabla a\right)
$$

with TRANSP calculated diffusion coefficients $D_{a}$ for a physical quantity $a$ such as particle density, heat etc.

TRANSP takes into the consideration multiple additional relations, such as an ideal gas equation of state for plasma particles, plasma angular momentum change due to the torque from neutral beam injection, an angular momentum balance equation, and magnetic field 
diffusion. There are several links posted on the TRANSP help webpage [29] for articles related to these aspects, such as [30] and others.

The general input values required for a TRANSP calculation involve the plasma current, the static electric potential, the plasma cross-section geometry, the power radiated from the plasma, the electron density and temperature profiles, the ion temperature profile, and the ion effective charge. As output one obtains the resistivity profile, the safety factor profile, the current density profile, the electric potential profile, the particle density profile, the particle flow and diffusion coefficients, the heat flow, the conductive heating, the emissivity profile etc.

The quantities calculated by TRANSP are flux-surface averaged, which may not be a very accurate representation of the emissivity when there exists a large population of trapped fast ions. Trapped ions are ions with a small velocity component parallel to the magnetic field. Due to the magnetic mirroring effect they stop moving along the magnetic field line in the high-field region of the magnetic field and reverse their drift direction. As a consequence, they are not traveling completely around the flux surface. (Figure 75)

The module NUBEAM, integrated in TRANSP, performs a time dependent Monte Carlo calculation of the slowing down of fast ions originating from the neutral beam. The typical time scale for collision processes between fast beam ions and bulk plasma particles is much shorter than the typical equilibrium evolution time scale of the bulk plasma. During the short time interval during which the deposition and slowing down of fast ions is calculated the bulk plasma properties are therefore assumed to be constant. The deposited quantities (energy, momentum, particles) are then passed to the TRANSP plasma model allowing it to evolve accordingly for a short time interval. After that the procedure is 
repeated. The emissivity is calculated on a two-dimensional grid in the poloidal cross section. This is the most important TRANSP output for comparison to our measured proton (or neutron) rates.
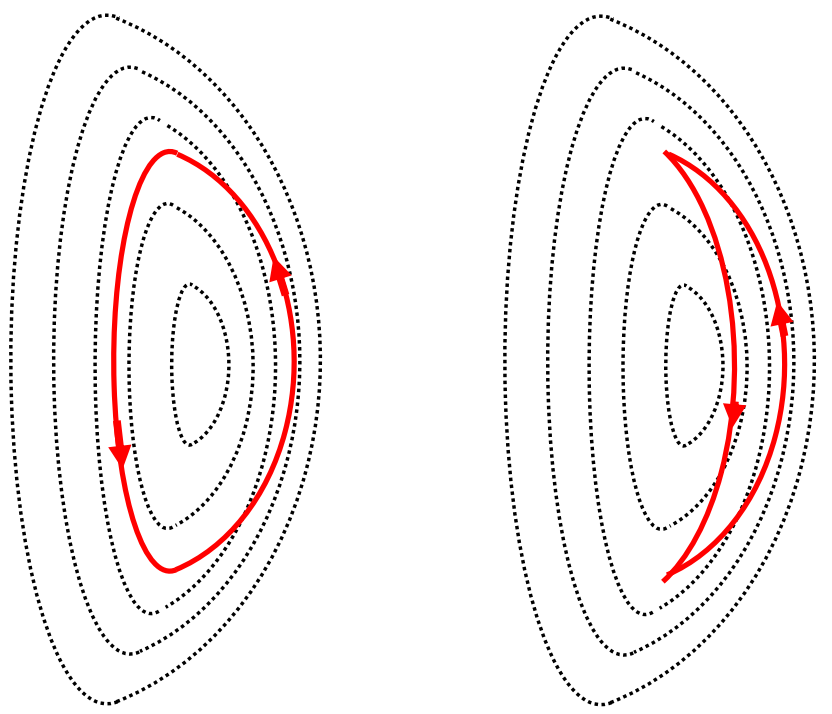

Figure 75. Passing (left) and trapped (right) fast ion gyrocenter motion for a poloidal cross-sectional projection. Dashed lines represent the equilibrium flux surfaces.

\subsection{Application of the Kick Model [25]}

Data analysis from previous experimental campaigns revealed a significant discrepancy between the detected and the calculated proton rates based on TRANSP/NUBEAM emissivity profiles, the detected rates were between 50 to 75 percent of the predicted values [24]. Specifically, for shots with plasma MHD activity a factor of two differences were observed. 


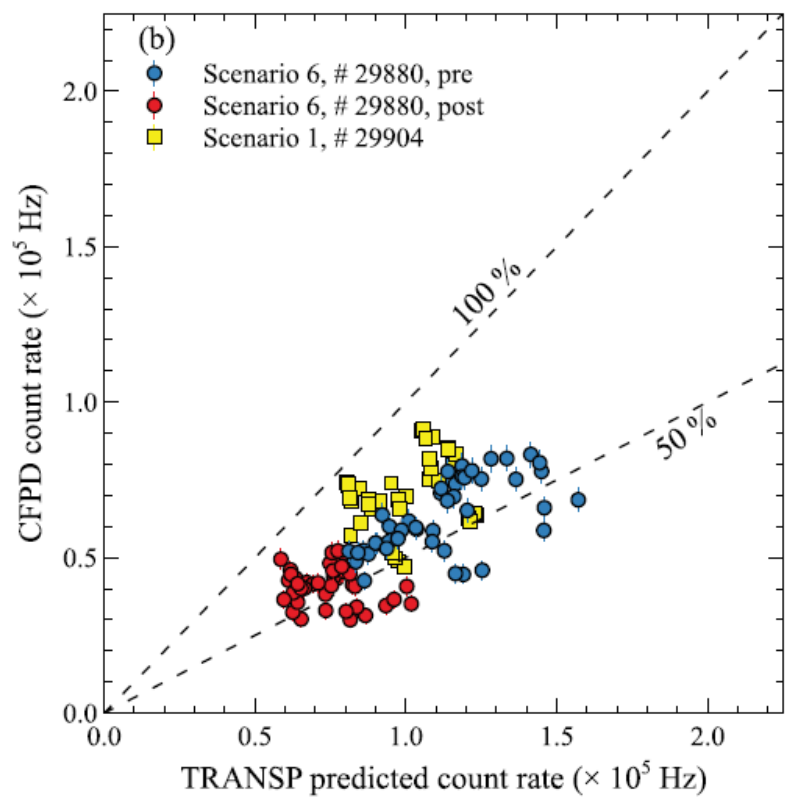

Figure 76. MAST 2013 experimental campaign Charged Fusion Products Diagnostic (CFPD) count rates comparison with TRANSP emissivity calculated rates [24]. Scenario 1 is a quiescent MHD plasma discharge, and Scenario 6 is a sawtooth instability plasma discharge.

To study this issue the so-called kick model by M. Podesta [25] was used to calculate the emissivity profiles impacted by MHD activities such as fishbone instabilities and TAE (Toroidal Alfven Eigenmodes) via the reduced fast ion transport model in the TRANSP code. The kick model uses spatial profile of a selected mode and calculates the kick probability matrix for fast ions, which describes the probability of a fast ion to receive a momentum kick from the MHD perturbation at the given particle location in phase space. Based on this matrix and the kick scaling amplitude, which is passed as a parameter to TRANSP, the fast ion transport simulation applies these kicks to the particles and thus models the perturbation's effect on the fast-ion distribution evolution and hence the emissivity profile. 

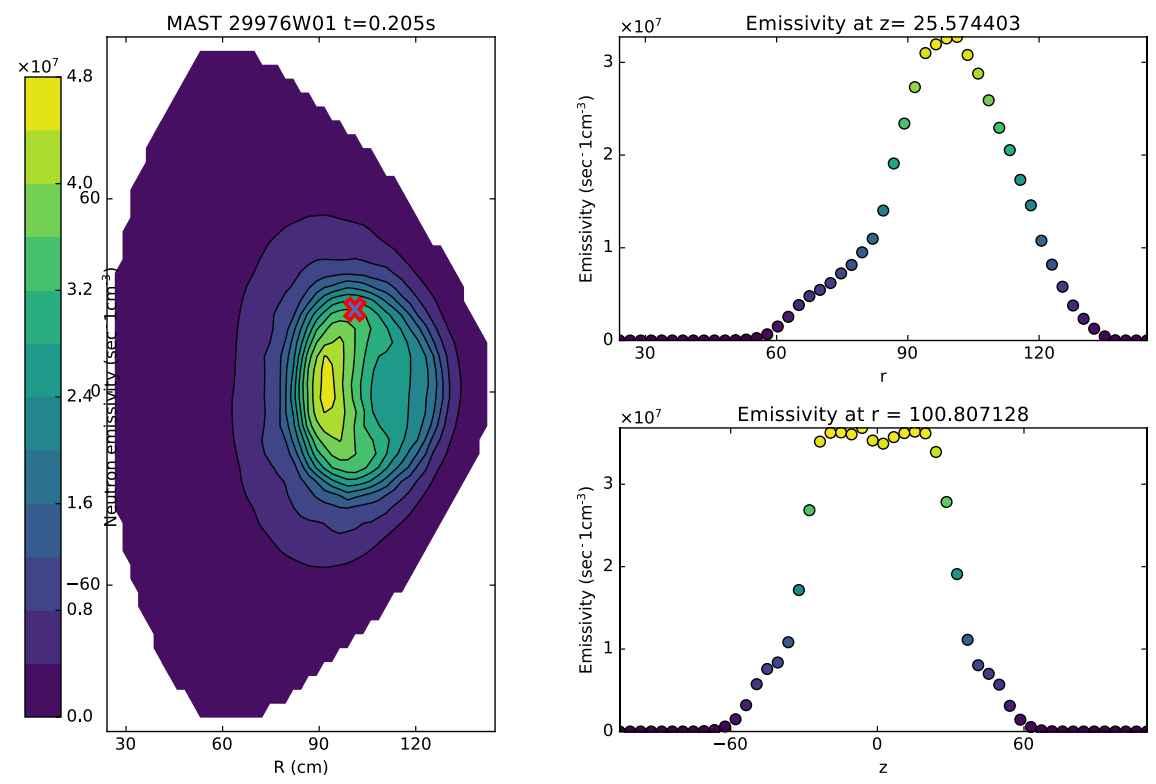

Figure 77. Plasma discharge (shot) 29976 emissivity profile where the Kick model was applied to the fast ion transport simulation in TRANSP. Upper right plot is the emissivity as a function of the radial coordinate for a fixed vertical position (horizontal slice at $z=25.6 \mathrm{~cm}$ ). The bottom right plot shows a slice of the emissivity at constant $r=100.8 \mathrm{~cm}$ as function of $z$. The red cross indicates the intersection point of the two slices (vertical and horizontal).

We calculated emissivity profiles for five different times during the plasma discharge.

Figure 77 shows the emissivity profile for $t=0.205 \mathrm{~s}$ with an unscaled kick amplitude for the TRANSP calculation. One can clearly see that the emissivity is not constant on a flux surface (see Figure 14 showing the typical flux surfaces for MAST). In Figure 78 the resulting emissivity profile is shown with a doubled kick amplitude. Compared to the profile with a smaller amplitude (Figure 77) the profile appears wider, less peaked in the radial direction while the change in the vertical direction is less apparent. 

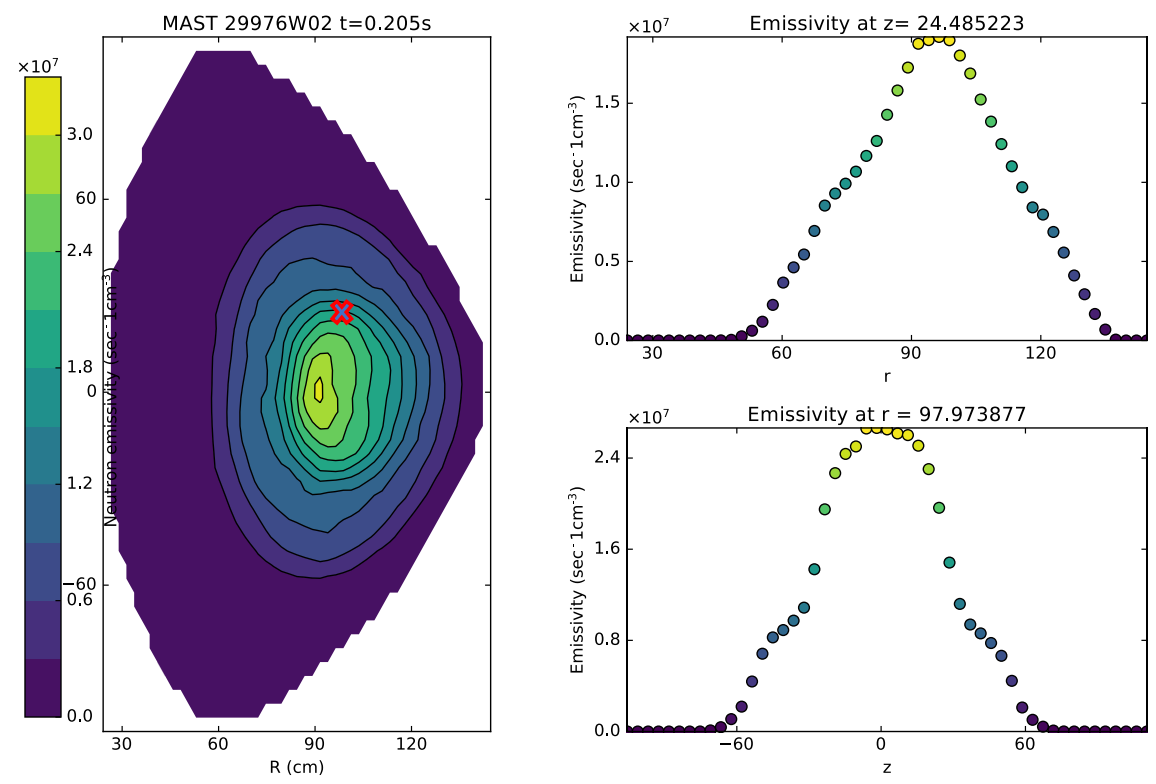

Figure 78. Shot 29976 emissivity profile where the Kick model was applied with doubled kick amplitudes scaling to the fast ion transport simulation in TRANSP. Upper right plot is the emissivity as a function of the radial coordinate for a fixed vertical position (horizontal slice at $z=25.6 \mathrm{~cm}$ ). The bottom right plot shows a slice of the emissivity at constant $r=$ $100.8 \mathrm{~cm}$ as function of $z$. The red cross indicates the intersection point of the two slices (vertical and horizontal).

The calculated model rates were compared to the experimental measured rates. Figure 80 shows an overview of the experimental rates for MAST shot 29976 during the full discharge time. Channel 0 data is not available for this shot presumably due to neutralbeam-spray particle overload. The same problem most likely affected Channel 1 after 0.19 s as well. Channel 3 is showing unexpectedly low rates due to a partial obstruction of its sightline. Calculated rates based on Kick model emissivity profiles with unscaled and doubled kick amplitudes are shown with circles and triangles for the five selected time points. The predicted rates with unscaled kick amplitudes appear to be roughly a factor of two higher than the measured rates. 
MAST Shot 29976

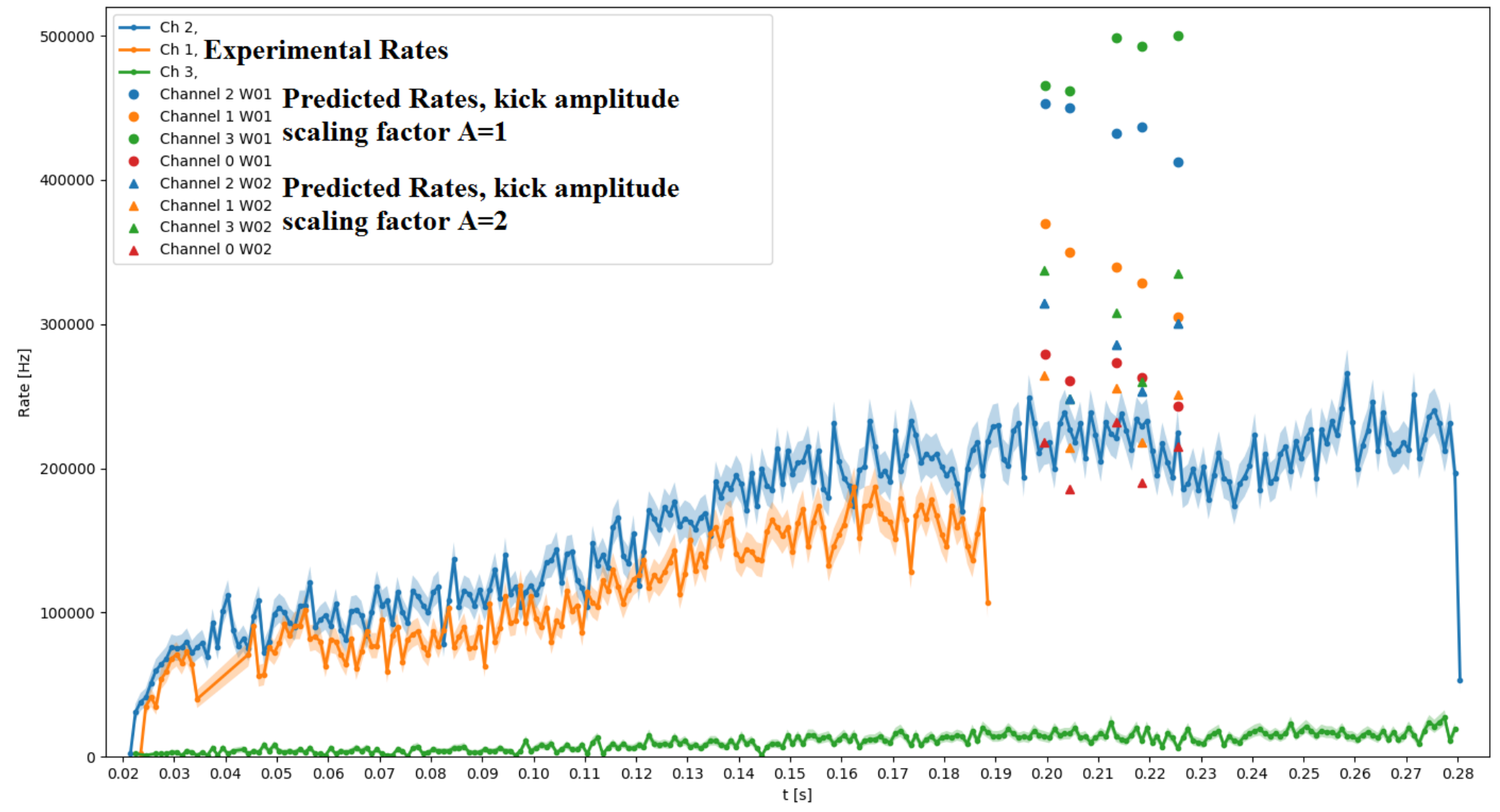

Figure 79. MAST shot 29976 measured and predicted rates with kick model TRANSP simulated emissivity profiles. Measured rates data is not available for Channel 0 (Detector 1) and Channel 1 (Detector 2) after 0.19 s presumably due to the neutral beam spray particle overload and of bad quality for Channel 3 (Detector 4) because of partial obstruction of the sightline. 
Focusing on Channel 2 data one can see (Figure 80) that with the introduction of the kick model the predicted rates can be brought closer to the measured values by changing the scaling factor of the kick amplitudes. The use of the kick model has just begun and is an ongoing project that is well beyond the scope of this work. While the modeled particle rates seem to agree better with the measured ones, other diagnostic data need also to be tested in order to evaluate the validity of this model. This was however the first time that proton and neutron rate data have been compared to TRANSP calculations including the kick model.

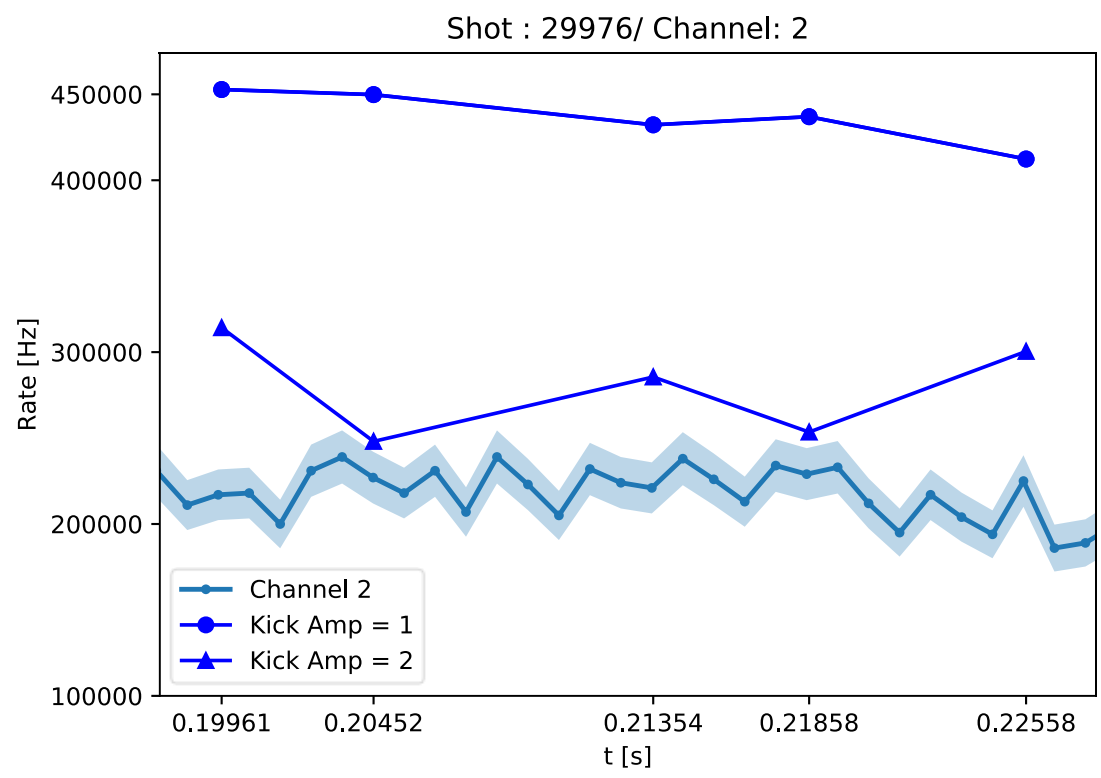

Figure 80. MAST shot 29976 Channel 2 measured proton rate and rate predicted with TRANSP simulated emissivity profile with a kick model applied and kick amplitude scaling factor set to 1 and 2 . 


\section{CHAPTER 8. CONCLUSION}

Within the scope of this dissertation, several important aspects of a plasma diagnostic with charged fusion-product particle detection were examined. The underlying properties of fusion reactions and plasma confinement principles were reviewed, and the analytical approach of finding the plasma equilibrium from the solution of the Grad-Shafranov equation was studied and implemented, with contribution of connecting the obtained solution to the real tokamak parameters. Analytically obtained magnetic field and current density profiles were compared to the corresponding outputs of the commonly used equilibrium solver EFIT.

A new design of the instrument was realized including a set of mechanical drawings produced at FIU followed by machining at a local contractor. The new system is much more flexible thanks to its modular design and it also allows an extension to a higher number of detectors. A removable collimation system for each detector housing makes it suitable for various plasma scenarios with increased injected beam power and the resulting higher particle flow rates. Detector orientation optimization was performed to achieve an optimal probing of the core plasma for various possible equilibrium magnetic field configurations. A new graphical user interface was developed for particle orbit calculations to simplify and speed up the optimization process. The orbit tracking code was modified to account for trajectory curvature inside the detector-collimator system and the effect on probing localization due to that effect was studied. 
A Monte Carlo based detector-collimator system acceptance calculation was implemented and the acceptance reduction due to the presence of a magnetic field was studied leading to the conclusion that it can be neglected at MAST-U as well as NSTX-U. The rate calculation method was discussed, analytically justified, and used for rate calculations based on the TRANSP simulated emissivity profiles. The results of these calculations were used to determine the optimal collimator openings for the new system including the necessary acceptance-reducing inserts for the previously used 4-channel system.

The kinematics of the fusion reaction was reviewed, and the proton energy spectrum was studied analytically using a reduced distribution function derivation [20]. A comparison to the Monte Carlo simulated spectrum showed the validity of the analytical approach and convoluted energy spectra were calculated for typical probing-trajectory and neutral-beam-energy distributions. The fusion reaction cross-sectional anisotropy was introduced and its effect on the predicted rate was calculated.

The data acquisition LabView code was developed for the new digitizer hardware and successfully tested. The data analysis code was revised and updated with database parameter storage and a graphical user interface was added.

TRANSP simulations with a kick model enhanced reduced fast ion transport code were performed and the output emissivity profiles were used to study the influence of plasma MHD perturbations on fast ion redistribution and reaction rates. 
Data taking for various plasma and neutral beam power conditions are planned to be carried out in 2020. Temperature measurement of the sensors installed in the probe head will be obtained and analyzed to determine the heat load on the detectors to judge the need for a detector cooling system. An energy resolution optimization will be attempted to achieve 100-150 keV energy resolution (FWHM) in order to measure the proton energy spectrum. The proton energy spectrum contains information about the velocity distribution of plasma and neutral beam ions. The fast ion thermalization processes and fast ion losses due to scattering or various other MHD instabilities can be studied using spectral data.

A detailed analysis of the future experimental data will allow us to determine absolute rates and extract time-dependent emissivity-profile data that can be compared to TRANSP calculations and other available diagnostic data. The new data will allow us to study the effects of plasma instabilities on fast ion redistribution, losses and thermalization processes. 


\section{LIST OF REFERENCES}

[1] U.S. Energy Information Administration, https://www.eia.gov/

[2] Nuclear fusion, https://en.wikipedia.org/wiki/Nuclear_fusion

[3] H.-S. Bosch and G.M. Hale 1992 Nucl. Fusion 32611

[4] Isenberg P. A., Jokipii J. R., Gradient and curvature drifts in magnetic fields with arbitrary spatial variation, Astrophysical Journal, Part 1, vol. 234, Dec. 1, 1979, p. 746-752

[5] J. A. Bittencourt, FUNDAMENTALS OF PLASMA PHYSICS. Springer Science + Business Media, LLC, 3rd Edition, 2004. 2

[6] http://www.ccfe.ac.uk/mast_upgrade_project.aspx

[7] https://www.iter.org/newsline/205/1017

[8] S. Li et. al. "Optimal Tracking for a Divergent-Type Parabolic PDE System in Current Profile Control", Abstract and Applied Analysis doi:10.1155/2014/940965

[9] L. Spitzer and R. Harm, Phys. Rev. 89, 977 (1953)

[10] Sawtooth Instability in Tokamak Plasmas Astrophysics and Space Science, 1997, Volume 256, Number 1-2, Page 177 R. J. Hastie

[11] Simulations towards the achievement of non-inductive current ramp-up and sustainment in the National Spherical Torus Experiment Upgrade - Scientific Figure on ResearchGate. Available from: https://www.researchgate.net/figure/Left-layout-of-the-beamlines-The-first-NBIhas-three-sources-at-tangency-radius-of-50_fig1_283478803

[12] Y.-K.M. Peng, The physics of spherical torus plasmas, Phys. Plasmas 7 (2000) 1681. 
[13] EUROfusion. Scheme of the NBI principle. https://www.eurofusion.org/wpcms/wp-content/uploads/2011/07/8c.jpg

[14] R. J. Goldston and P. H. Rutherford. Introduction to Plasma Physics. Institute of Physics Publishing, 1995. 16, 17, 104, 105, 106, 107, 108, 109, 110

[15] L. S. Solov'ev, Sov. Phys. JETP 26, 400 (1968), THE THEORY OF HYDROMAGNETIC STABILITY OF TOROIDAL PLASMA CONFIGURATIONS

[16] Jeffrey Freidberg, Plasma Physics and Fusion Energy. Cambridge University Press, 2007. xii, 4, 5, 6, 8, 9, 10, 11, 12, 13, 104

[17] Reconstruction of current profile parameters and plasma shapes in tokamaks, L.L. Lao et al 1985 Nucl. Fusion 251611

[18] Netepenko A., Boeglin W.U., Darrow D.S., Ellis R. and Sibilia M.J. 2016 Rev. Sci. Instrum. 87 11D805

[19] Perez, Ramona V., "A Charged Fusion Product Diagnostic for a Spherical Tokamak" (2015). FIU Electronic Theses and Dissertations. Paper 2233. http://digitalcommons.fiu.edu/etd/2233

[20] W.W. Heidbrink, Nucl. Instrum. Meth. A236 (1985) 380

[21] BROWN, R.E., JARMIE, N., Phys. Rev., C 41 (1990) 1391

[22] J. Boris, in Proceedings of the Fourth Conference on Numerical Simulation of Plasmas (Naval Research Laboratory, Washington D. C., 1970), p. 3.

[23] Application of Liouville's Theorem to Electron Orbits in the Earth's Magnetic Field W. F. G. Swann Phys. Rev. 44, 224

[24] M. Cecconello et al 2019 Nucl. Fusion 59016006

[25] M Podestà et al 2017 Plasma Phys. Control. Fusion 59095008 
[26] Review of Scientific Instruments 61, $3262 \quad$ (1990); https://doi.org/10.1063/1.1141608 Published Online: 09 September 1998

[27] J.P.H.E. Ongena, M. Evrard \& D. McCune (1998) Numerical Transport Codes, Fusion Technology, 33:2T, 181-191, DOI: 10.13182/FST98-A11947009

[28] R.J. Hawryluk "An empirical approach to tokamak transport", in Physics of Plasma close to Thermonuclear Conditions, Proc. of the Course held in Varenna 1979 (CEC Brussel, 1980), Vol.1, 19-46

[29] https://w3.pppl.gov/ pshare/help/transp.htm

[30] Goldston, R.J., McCune, D., Towner, H.H., Davis, S.L., Hawryluk, R.J., \& Schmidt, G.L. (1981). New techniques for calculating heat and particle source rates due to neutral beam injection in axisymmetric tokamaks. 
VITA

\section{ALEXANDER NETEPENKO}

\section{EDUCATION}

$2002-2008$

M.S., Physics

Novosibirsk State University,

Novosibirsk, Russian Federation

$2010-2011$,

Ph.D., Physics

$2014-2019$

Florida International University

Miami, Florida

\section{PUBLICATIONS AND PRESENTATIONS}

A. Netepenko, W. U. Boeglin, D. S. Darrow, R. Ellis, and M. J. Sibilia. Plasma diagnostics in spherical tokamaks with silicon charged-particle detectors. Rev. Sci. Instrum. 87, 11D805 (2016).

Y. Alexahin, E. Gianfelice-Wendt, A. Netepenko. Conceptual design of the muon collider ring lattice. Proc. IPAC'10, paper TUPEB021 (2010).

A. Netepenko, Muon Collider Ring Lattice Design, Jefferson Lab, Beam Physics and CASA Seminars, Newport News, VA (2010). 